GUSTAVO HENRIQUE MURIEL ZANON

Técnicas de compressão de chaves para criptossistemas baseados em isogenias

São Paulo 



\section{Técnicas de compressão de chaves para criptossistemas baseados em isogenias}

\section{Versão Original}

Dissertação apresentada à Escola Politécnica da Universidade de São Paulo para a obtenção do título de Mestre em Ciências.

Área de Concentração: Engenharia de Computação

Orientador: Prof. Dr. Marcos Antonio Simplicio Junior

São Paulo

2021 
Autorizo a reprodução e divulgação total ou parcial deste trabalho, por qualquer meio convencional ou eletrônico, para fins de estudo e pesquisa, desde que citada a fonte.

\section{Catalogação-na-publicação}

\section{Zanon, Gustavo Henrique Muriel}

Técnicas de compressão de chaves para criptossistemas baseados em isogenias / G. H. M. Zanon -- São Paulo, 2021.

$96 \mathrm{p}$.

Dissertação (Mestrado) - Escola Politécnica da Universidade de São Paulo. Departamento de Engenharia de Computação e Sistemas Digitais.

1.Criptologia 2.Isogenias 3.Algoritmos I.Universidade de São Paulo. Escola Politécnica. Departamento de Engenharia de Computação e Sistemas Digitais II.t. 


\section{Resumo}

A criptografia baseada em isogenias supersingulares constitui uma das famílias mais recentes dentre as propostas resistentes a ataques com computadores quânticos. Uma característica interessante é a sua baixa ocupação de banda, viabilizada pela natureza intrínseca do problema computacional, em comparação a outros protocolos de acordo de chave, e potencializada pela possibilidade de compressão ulterior de chaves. No entanto, a compressão e correspondente descompressão introduzem uma sobrecarga significativa no custo geral de processamento, apesar de progressos recentes. Neste trabalho, abordam-se os principais gargalos de processamento envolvidos na compressão e descompressão de chaves e sugerem-se técnicas eficientes para todas as etapas desse processo, a saber, geração eficiente das bases de torção, cálculo eficiente de logaritmos discretos e cálculo eficiente de emparelhamento supersingulares. Quando utilizados em conjunto, essas técnicas produzem ganhos observados de até três ordens de grandeza em relação aos melhores resultados de técnicas anteriormente propostas na literatura.

Palavras-chave: algoritmos eficientes, criptografia pós-quântica, criptologia, isogenias supersingulares, protocolos criptográficos. 



\section{Abstract}

Supersingular isogeny-based cryptography is one of the most recent families among proposals resistant to attacks with quantum computers. An interesting feature is the comparatively low bandwidth occupation, made possible by the intrinsic nature of the computational problem, compared to other key agreement protocols, and enhanced by the possibility of further key compression. However, compression and corresponding decompression introduce a significant overhead to the overall processing cost, despite recent progress. In this work, the main processing bottlenecks involved in the compression and decompression of keys are addressed and efficient techniques are suggested for all stages of this process, namely, efficient generation of the torsion bases, efficient calculation of discrete logarithms and efficient calculation of supersingular pairing are suggested. When used together, these techniques produce observed gains of up to three orders of magnitude in relation to the best results of techniques previously proposed in the literature.

Keywords: efficient algorithms, post-quantum cryptography, cryptology, supersingular isogenies, cryptographic protocols. 



\section{Lista de ilustrações}

Figura 1 - Curva de Weierstrass $y^{2}=x^{3}-8 x+4$ sobre $\mathbb{R} \ldots \ldots \ldots 32$

Figura 2 - Curva $y^{2}=x^{3}-8 x+4$ sobre $\mathbb{F}_{11} \ldots \ldots \ldots \ldots$

Figura 3 - Inverso de um ponto da curva. . . . . . . . . . . . . . . . 34

Figura 4 - Soma de pontos (regra da secante) . . . . . . . . . . . . . 34

Figura 5 - Duplicação de um ponto (regra da tangente) . . . . . . . . . . . 35

Figura 6 - Curva degenerada $y^{2}=x^{3}-3 x-2$, singular em $S=(-1,0) \ldots \ldots 36$

Figura 7 - Curva degenerada $y^{2}=x^{3}-3 x+2$, singular em $S=(1,0) \ldots \ldots$. . . . 36

Figura 8 - Curva degenerada $y^{2}=x^{3}$, singular em $S=(0,0) \ldots \ldots \ldots \ldots$

Figura 9 - Curva $y^{2}=x^{3}+x-1$ sobre $\mathbb{F}_{7}$ gerada por $G=(1,1) \ldots \ldots \ldots$

Figura 10 - Curva supersingular $y^{2}=x^{3}+1$ sobre $\mathbb{F}_{5} \ldots \ldots \ldots \ldots$

Figura 11 - Curva de Montgomery $5 y^{2}=x^{3}+10 x^{2}+x \ldots \ldots$. . . . . . . . 39

Figura 12 - Esquematização do protocolo de troca de chaves ECDH. . . . . . . . . . . . 44

Figura 13 - Esquematização do protocolo de troca de chaves SIDH. . . . . . . . . . . . 49

Figura 14 - O conjunto dos $37 \mathrm{j}$-invariantes de curvas supersingulares em $\mathbb{F}_{431^{2}} \ldots \ldots$

Figura 15 - Exemplo de um triângulo equilátero discreto $\Delta . \quad \ldots \ldots$. . . . . . . . . . 74

Figura 16 - Caminhar para a direita em $\Delta$ equivale a uma exponenciação e para a direita, remover o $(j+k)$-ésimo dígito do expoente do vértice de origem. . . . . . 75

Figura 17 - Exemplo de caminho em $\Delta$ traçado pela estratégia ingênua. . . . . . . . . . 76

Figura 18 - Exemplo de caminho em $\Delta$ traçado pela estratégia ótima. . . . . . . . . . . 76 



\section{Lista de tabelas}

Tabela 1 - Tipos de notações: aditiva e multiplicativa . . . . . . . . . . . . 28

Tabela 2 - Discriminante de uma curva elíptica . . . . . . . . . . . 35

Tabela 3 - Custo do laço de Miller binário (a razão assume $\mathbf{s} \approx 0.8 \mathbf{m}$ e ignora $\mathbf{a}$ ). . . . 69

Tabela 4 - Custo do laço ternário de Miller (a razão assume $\mathbf{s} \approx 0.8 \mathbf{m}$ e ignora $\mathbf{a}$ ). . . . 72

Tabela 5 - Custos do cálculo do logaritmo discreto (assumindo $\mathbf{s} \approx 0.8 \mathbf{m}$ ) . . . . . . 77

Tabela 6 - Benchmark da geração da base de $3^{\mathbf{n}}$-torção. . . . . . . . . . . . . . 82

Tabela 7 - Benchmark da geração da base de $2^{\mathbf{m}}$-torção $\ldots \ldots \ldots$

Tabela 8 - Resultados do obtidos por por etapa do protocolo. . . . . . . . . . . 83

Tabela 9 - Impactos dos algoritmos de (des)compressão no SIKE. . . . . . . . . . 83 



\section{Lista de abreviaturas e siglas}

$\begin{array}{ll}\text { ARM } & \text { Acorn RISC Machine } \\ \text { BDHP } & \text { Bilinear Diffie-Hellman Problem } \\ \text { CDHP } & \text { Computational Diffie-Hellman Problem } \\ \text { CSIDH } & \text { Commutative Supersingular Isogeny Diffie-Hellman } \\ \text { DDHP } & \text { Decisional Diffie-Hellman Problem } \\ \text { ECDH } & \text { Elliptic Curve Diffie-Hellman } \\ \text { GDHP } & \text { Gap Diffie-Hellman Problem } \\ \text { GMP } & \text { GNU Multiple Precision Arithmetic Library } \\ \text { NIST } & \text { National Institute of Standards and Technology } \\ \text { NTRU } & N^{\text {th }} \text { Degree Truncated Polynomial Ring } \\ \text { SIDH } & \text { Supersingular Isogeny Diffie-Hellman } \\ \text { SIKE } & \text { Supersingular Isogeny Key Encapsulation }\end{array}$





\section{Sumário}

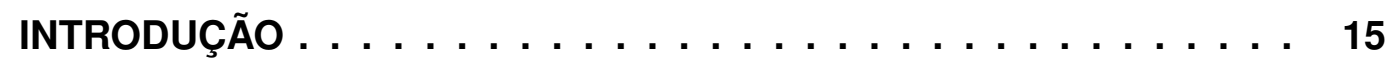

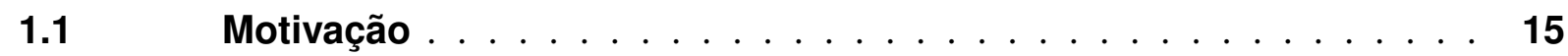

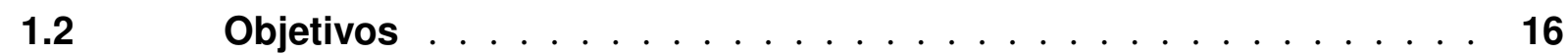

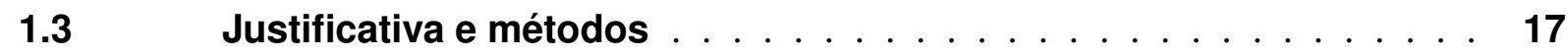

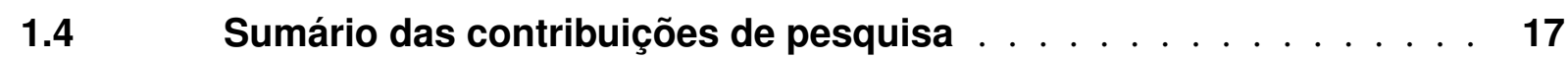

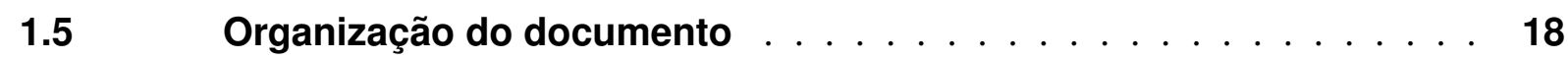

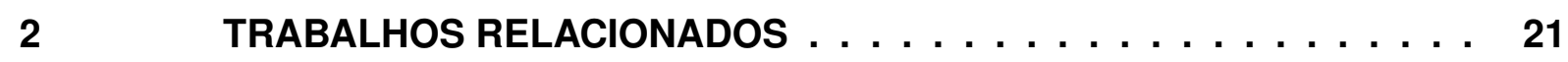

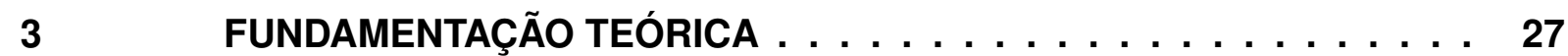





3.2.1 Modelo de Weierstrass . . . . . . . . . . . . . . . . . . . . 32

3.2.1.1 Lei de grupo de Weierstrass (secantes e tangentes) . . . . . . . . . . . 33

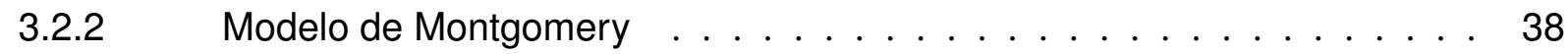

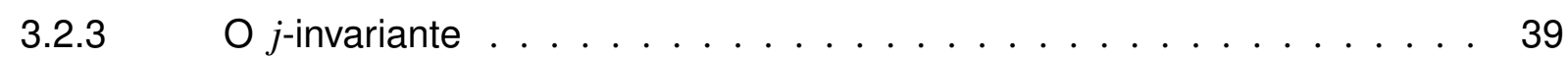

3.2.4 Emparelhamentos bilineares . . . . . . . . . . . . . . . . . . . 40

3.2.5 A técnica Elligator $2 \ldots \ldots \ldots \ldots$. . . . . . . . . . . . 42

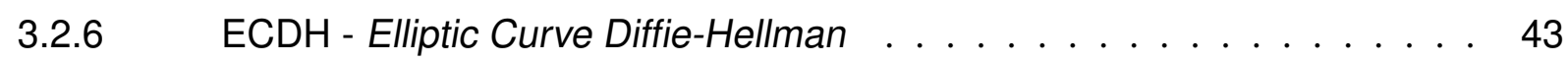

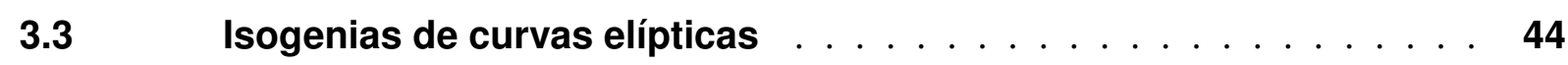

3.3.1 Cálculo de isogenias de grau suave . . . . . . . . . . . . . . . 45

3.3.2 Fórmulas de Vélu . . . . . . . . . . . . . . . . . . . . . 47

3.3.3 SIDH - Supersingular Isogeny Diffie-Helman . . . . . . . . . . . . . 48

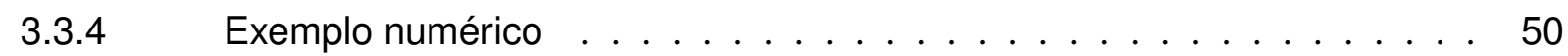

3.3.5 O problema da compressão de chaves . . . . . . . . . . . . 51

4 COMPRESSÃo EFICIENTE DE CHAVES ISÓGENAS . . . . . . . 53

$4.1 \quad$ Notação e convenções . . . . . . . . . . . . . . . . 53

4.2 Decomposição reversa de bases . . . . . . . . . . . . 53

$4.3 \quad$ Geração de bases emaranhadas . . . . . . . . . . . . . 55

4.3.1 Evitando a multiplicação por cofator . . . . . . . . . . . . . 58

$4.4 \quad$ Geração de bases em $E\left[3^{n}\right] \ldots \ldots \ldots$. . . . . . . . . 60

4.4.1 Elligator compartilhado e descompressão mais rápida . . . . . . . . . . 62

4.4.2 O Elligator compartilhado em bases emaranhadas . . . . . . . . . . 63

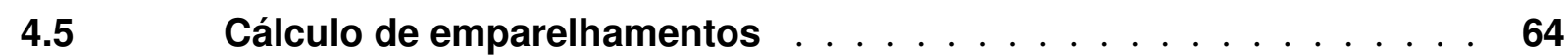

4.5.1 Emparelhamentos de ordem binária . . . . . . . . . . . . 66 
4.5.1.1 Emparelhamentos numa base emaranhada . . . . . . . . . . . . . . . 68

4.5.2 Emparelhamentos de ordem ternária . . . . . . . . . . . . . 69

$4.6 \quad$ Cálculo de logaritmos discretos . . . . . . . . . . . . . . . 72

4.6.1 O custo do cálculo do logaritmo discreto . . . . . . . . . . . . 76

4.6.2 Pohlig-Hellman aprimorado para $w$ genérico $\ldots \ldots \ldots$. . . . . . 78

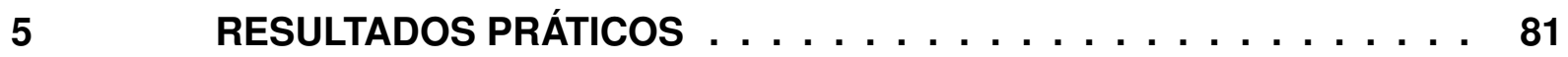

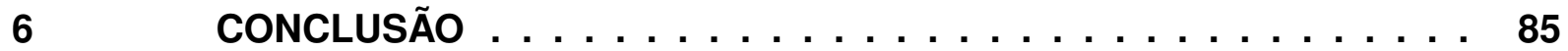

$6.1 \quad$ Sugestões para pesquisas futuras $\ldots \ldots \ldots \ldots$

REFERÊNCIAS . . . . . . . . . . . . . . . . . . 87

$\begin{array}{ll}\text { APÊNDICES } & 93\end{array}$

APÊNDICE A - ARITMÉTICA DE MONTGOMERY . . . . . . . . . . . 95 


\section{Introdução}

Pode-se argumentar que o acordo de chaves é o protocolo criptográfico quantitativamente mais importante, pois hoje em dia quase todos serviços online são feitos através do protocolo https, como comércio eletrônico, acesso a e-mail pessoal ou comercial, ou ainda por protocolos similares, como a criptografia ponta-a-ponta empregada por aplicativos como WhatsApp e Signal. Dado que esses protocolos são feitos através de acordo de chaves, justifica-se sua importância em termos de volume.

Por muito tempo a segurança foi negligenciada em detrimento de desempenho, porém com o passar do tempo foi reavaliada como fator sumamente relevante a ponto de se tornar o padrão de acesso, seja porque as máquinas (cliente e servidor) estão mais baratas, seja porque a cultura dos provedores de serviços online deslocou-se para uma preocupação maior com segurança em vez de desempenho. Evidência disso encontra-se no acesso a sites de serviços como e-commerce, a fim de proteger o consumidor de ataques a transações, e também a sites institucionais, como por exemplo os serviços oferecidos pela Google, que agora estão sendo feitos por via de regra via https (LIDZBORSKI, 2014). Ainda, a Google adotou o padrão de exibir resultados de buscas com a versão https de páginas que oferecerem esse tipo de segurança (BAHAJJI, 2015).

Em paralelo, nota-se nos últimos anos uma preocupação crescente com ataques montados com o auxílio de computadores quânticos, que contornam eficientemente todas as proteções oferecidas pela criptografia assimétrica convencional (SHOR, 1994). Um exemplo disto está nas experiências conduzidas pela Google em seu navegador Chrome para Desktops, onde parte das comunicações seguras eram feitas através do acordo de chaves pós-quântico NewHope (BRAITHWAITE, 2016)). Essa preocupação é inteiramente justificada face aos avanços recentes na construção de computadores quânticos efetivos: empresas como IBM, Intel, Microsoft e Google têm investido recursos em busca de um computador quântico de propósito geral. Também computadores quânticos com propósitos mais limitados como é o caso do D-Wave, que, apesar de limitado, é capaz de resolver eficientemente o problema da fatoração inteira em que se apoia o criptossistema RSA tradicional. Tendo isso em vista, um protocolo moderno de acordo de chaves deve também resistir a ataques quânticos.

\subsection{Motivação}

Entre o final de 2015 e início de 2016, o Instituto Nacional de Padrões e Tecnologias dos Estados Unidos (NIST) iniciou um esforço conjunto entre pesquisadores através de uma competição com a finalidade de definir algoritmos a serem utilizados como padrões de segurança no cenário pós-quântico. Surpreendentemente, candidatos a protocolos de acordo de chaves 
resistentes a ataques quânticos apoiam-se em um número reduzido de problemas computacionais: como reticulados (ALKIM et al., 2016), códigos corretores de erros (ARAGON et al., 2017) e isogenias entre curvas elípticas; Há também outros problemas, porém de âmbito muito mais restrito, como assinaturas baseadas em hash, sistemas multivariados quadráticos e protocolos de identificação baseados em núcleos e percéptrons permutados.

Dentre as famílias de problemas computacionais citadas, a mais recente é a baseada em isogenias, que possuí como principal vantagem o tamanho reduzido de chaves em relação aos demais candidatos, com possibilidade de se tornarem ainda menores através de técnicas de compressão de chaves. Entretanto, as técnicas utilizadas para a (des)compressão de chaves resultam em perdas significativas de desempenho (AZARDERAKHSH et al., 2016; COSTELLO et al., 2017). Outro contraponto em relação aos criptossistemas baseados em isogenias surge exatamente pelo fato de serem propostas recentes e não possuírem a mesma maturidade tecnológica dos concorrentes. Para que essa maturidade seja adquirida, muita pesquisa deverá ser feita sob essa área, assim como as alternativas baseadas em códigos, reticulados e semelhantes começaram ineficientes e ganharam melhorias ao longo de anos ou décadas.

\subsection{Objetivos}

O trabalho aqui delineado propõe melhorias tecnológicas para tornar madura a família de algoritmos baseados em isogenia de acordo com as métricas de ocupação de banda, desempenho e resistência a ataques de ambos computadores clássicos e quânticos.

O escopo dessa trabalho limita-se em investigar técnicas para melhorar o criptossistema SIKE, uma vez que foi submetido à padronização do NIST e portanto acredita-se que tenha segurança pós-quântica. Esse criptossistema reduz a ocupação de banda através da compressão de chaves ao custo de desempenho, uma vez que esse processo é computacionalmente intensivo.

Esta pesquisa de mestrado busca investigar novos algoritmos eficientes para compressão de chaves de modo que se possa reduzir ocupação de banda sem impacto significativo de desempenho. Assim, pode-se definir uma constante entre tempo e banda de tal forma que, ao se obter uma economia de metade da banda, não mais do que o dobro de processamento seja gasto como consequência. Como resultado, estima-se que o tempo gasto com compressão não seja superior ao tempo gasto na utilização das chaves.

Por se tratar de uma literatura recente, há pouco material disponível em português sobre o assunto e espera-se que esta dissertação forneça um material auto-contido na medida do possível que supra essa necessidade. 


\subsection{Justificativa e métodos}

A notável escassez de problemas computacionais capazes de sustentar as propriedades de segurança de criptossistemas efetivos cria a necessidade de não apenas investigar a resistência desses esquemas (diante de computadores clássicos e quânticos), mas também de otimizar os poucos sistemas conhecidos segundo as principais métricas de custo.

Tratando-se de protocolos de acordos de chaves criptográficas para sessões online, as métricas naturais são:

- Ocupação de banda.

- Velocidade de processamento;

Métricas específicas de aplicações particulares como redes de sensores sem fio podem incluir eficiência energética também, mas tipicamente essas métricas adicionais são correlacionadas com as métricas principais (por exemplo, o consumo de energia é comumente proporcional, nas plataformas típicas de redes de sensores sem fio, aos tempos de processamento e/ou à ocupação de banda).

Contudo, a característica mais distintiva e marcante de criptossistemas isógenos em comparação com outras propostas pós-quânticas (por assim dizer, o seu sales pitch) é a banda potencialmente reduzida que ocupa, menor que qualquer outra família de criptossistemas pósquânticos na literatura e apenas pouco maior que a banda ocupada por criptossistemas convencionais (suscetíveis a ataques quânticos). Por essa razão, esses esquemas são a opção mais óbvia para cenários onde a ocupação de banda é um gargalo crítico, e isso torna a métrica da ocupação de banda a mais crucial para esses esquemas (sem deixar de lado a velocidade de processamento, que ainda é importante embora não seja o mais central).

Isso tudo justifica a investigação de métodos para reduzir a ocupação de banda em sistemas isógenos supersingulares, mantendo a eficiência de processamento tão alta quanto possível para uma largura específica de banda, que é exatamente o objetivo deste trabalho.

\subsection{Sumário das contribuições de pesquisa}

Cada um dos resultados obtidos pela pesquisa realizada constitui pequenas melhorias complementares. Esses resultados são sumarizados a seguir:

- Geração eficiente de bases de torção.

- Assumindo os parâmetros usuais $\ell_{\mathrm{A}}=2, \ell_{\mathrm{B}}=3$, melhora-se a geração de base para $E\left[2^{m}\right]$ e $E\left[3^{n}\right]$. O algoritmo denominado geração de bases emaranhadas proposto para gerar bases de $2^{m}$-torção é aproximadamente $15.9 \times$ vezes mais rápido que a 
geração usual apresentada no trabalho anterior e tem aplicações não apenas em troca de chaves, mas também em funções hash baseadas em isogenias (DOLISKANI; PEREIRA; BARRETO, 2017). Para $3^{n}$-torção, observa-se que o algoritmo ingênuo é mais eficiente (tanto na teoria quanto na prática) do que a versão explícita 3-descenso de (SCHAEFER; STOLL, 2004) usado por Costello et al..

- Introduz-se a técnica Elligator compartilhado para acelerar ainda mais a construção da base de torção durante a descompressão, que permite uma geração de base ternária 1.5 - 2.8× mais rápida quando comparada com a técnica de geração simples anterior. Quando a nova geração de base emaranhada é acoplada ao Elligator compartilhado, as melhorias são ainda mais significativas, chegando a ser 29.9× mais rápido.

- Cálculo eficiente de logaritmos discretos.

- Inspirado pelo método de estratégia ótima de De Feo et al para calcular isogenias de grau suave (DeFEO; JAO; PLÛT, 2014), propõe-se um algoritmo para calcular logaritmos discretos no $\mu_{\ell^{n}}$ dado um método eficiente para calcular logaritmos discretos em $\mu_{\ell}$ onde $\ell$ é um primo pequeno, ou mais geralmente, um algoritmo para computar logaritmos discretos no grupo $\mu_{\left(\ell^{w}\right)^{n / w}}$ quando $w \mid n$, dado um método eficiente para calcular logaritmos discretos em $\mu_{\ell^{w}}$.

- Descreve-se como calcular Pohlig-Hellman no grupo $\mu_{\ell^{n}}$ a partir de uma adaptação da estratégia ótima de percurso, dado um método eficiente para computar logaritmos discretos no grupo $\mu_{\ell^{w}}$ quando $w \nmid n$.

- Cálculo eficiente de emparelhamentos supersingulares.

- Introduz-se a técnica de decomposição reversa de base, que combinado com as melhorias anteriores, permite otimizações adicionais de compressão e descompressão. Por exemplo, cada parte só precisa computar 4 emparelhamentos em vez de 5 . Além disso, duas multiplicações custosas pelo cofator $3^{n}$ são poupadas durante a compactação do Bob e uma multiplicação pelo cofator $3^{n}$ é poupada durante a descompactação da Alice.

- As formas especiais de pares de pontos gerados como bases emaranhadas e a existência de um subcorpo descartado por (COSTELLO et al., 2017) para otimizar o emparelhamento Tate foram exploradas. Com isso, atinge-se uma melhoria de $1.4 \times$ para a fase de emparelhamento sobre os algoritmos usados por (COSTELLO et al., 2017) para combinações binárias e ternárias.

\subsection{Organização do documento}

O Capítulo 2 deste documento apresenta uma revisão bibliográfica dos trabalhos relevantes relacionados até o presente momento. 
O Capítulo 3 fornece a base teórica para o entendimento das contribuições e discussões que serão apresentadas nos capítulos seguintes.

O Capítulo 4 apresenta as contribuições originais obtidas durante a fase de pesquisa.

O Capítulo 5 expõe os resultados práticos obtidos a partir das contribuições apresentadas.

O Capítulo 6 discute os resultados das contribuições e seus impactos frente a outros trabalhos. 



\section{Trabalhos relacionados}

Em 1994, quando Peter Shor publicou seu trabalho apresentando algoritmos que são capazes de resolver os problemas de fatoração e do logaritmo discreto em tempo polinomial em computadores quânticos (SHOR, 1994), tais computadores eram considerados uma realidade distante de ser alcançada e visto por muitos como puramente teóricos. Entretanto, caso viessem a se tornar práticos, seriam capazes de quebrar esquemas criptográficos cuja segurança está baseada na intratabilidade desses problemas por computadores clássicos, como é o caso do RSA e das curvas elípticas, largamente utilizados pela indústria em protocolos de assinatura e troca de chaves. A partir disso, criou-se a distinção entre criptossistemas clássicos e criptossistemas pós-quânticos, o qual acredita-se serem resistente a ataques de computadores quânticos.

A primeira proposta de um criptossistema baseado em isogenias, feita por Couveignes em 1997, descreveu um protocolo não interativo de troca de chaves, onde o espaço de chaves públicas é igual ao conjunto de $\mathbb{F}_{q}^{-}$classes de isomorfismo de curvas elípticas ordinárias sob $\mathbb{F}_{q}$, cujo anel de endomorfismo é uma dada ordem $O$ em um corpo quadrático imaginário com traço de Frobenius definido. Como a classe de grupos ideais $c l(O)$ age transitivamente sob $\mathbb{F}_{q}^{-}$ através da aplicação de isogenias, Couveignes observou que a comutatividade de $\operatorname{cl}(O)$ permite uma troca de chaves no estilo Diffie-Hellman. Essa proposta ficou em âmbito privado após ter sido rejeitada e tornou-se pública em 2006 (COUVEIGNES, 1997), porém durante esse período o método descrito foi redescoberto por Rostovtsev e Stolbunov de forma independente em 2004 (ROSTOVTSEV; STOLBUNOV, 2004; STOLBUNOV, 2010). O melhor algoritmo conhecido para resolver o problema de calcular isogenias em curvas elípticas ordinárias em um computador clássico possui tempo exponencial e foi proposto por Galbraith e Stolbunov (GALBRAITH; STOLBUNOV, 2011), entretanto, em 2010, Childs, Jao e Soukarev mostraram que quebrar o esquema Couveignes-Rostovtsev-Stolbunov é o mesmo que resolver uma instância do problema abeliano de deslocamento escondido, o qual possui algoritmos quânticos de ordem de complexidade subexponencial (CHILDS; JAO; SOUKHAREV, 2014). Como esse ataque depende do fato de que $c l(O)$ é comutativo, e indiretamente de que $O$ é comutativo, Jao e De Feo propuseram em 2011 o uso de curvas elípticas supersingulares, cujo anel completo de endomorfismos é uma ordem em uma álgebra de quaternion, que é não-comutativo. O esquema de acordo de chave resultante é atualmente conhecido como SIDH (Supersingular Isogeny DiffieHellman) (JAO; DeFEO, 2011; DeFEO; JAO; PLÛT, 2014). Em 2014, Azarderakhsh, Fishbein e Jao apresentaram uma implementação em linguagem $C$ empregando técnicas de otimização em assembly para arquiteturas ARMv7 e x86_64 (AZARDERAKHSH; FISHBEIN; JAO, 2014) que resultaram em um ganho de desempenho entre $18 \%$ e $26 \%$ sob o trabalho original do SIDH.

Em meados de 2015, o crescente investimento e avanços tecnológicos na área de computação quântica realizados por empresas como Google (KELLY; BARENDS; FOWLE, 2015), IBM 
(IBM, 2015), Intel (INTEL, 2015) e Microsoft (SVORE, 2015), tornou computadores quânticos uma realidade mais próxima de ser alcançada. Frente a essa ameaça, ainda em 2015 o Instituto Nacional de Padrões e Tecnologias dos Estados Unidos (NIST) se voltou à academia e indústria, para que uma discussão sobre uma futura possível padronização de algoritmos criptográficos resistentes a ataques quânticos fosse feita (NIST, 2015). No início de 2016 publicou um documento (CHEN et al., 2016) abordando a necessidade de transicionar os criptossistemas atuais para alternativas pós-quânticas, juntamente com planos delineados para "iniciar um esforço de padronização em criptografia pós-quântica". Com essa motivação, o interesse nas diferentes famílias de criptossistemas pós-quânticos cresceu e em especial em isogenias por:

- ser a família mais recente e portanto com maior potencial de ser explorada;

- possuírem menor tamanho de chaves em relação às alternativas, em especial ao considerar técnicas de compactação de chaves;

- serem construídas sob o arcabouço de curvas elípticas, bem conhecidas tanto pela academia quanto pela indústria;

- possuírem baixo desempenho, com potenciais otimizações a serem feitas.

Em 2016, Costello, Longa e Naehrig apresentaram uma nova implementação do SIDH em C com otimizações em assembly (COSTELLO; LONGA; NAEHRIG, 2016). Apesar de rodar em tempo constante e ser resistente a ataques de canal colateral de cache e de tempo, a implementação para arquitetura x64 obteve um ganho de desempenho de aproximadamente 2.9x vezes com relação à implementação acima para níveis de segurança quântica de 128 e clássica de 192 bits. As melhorias obtidas se deram através das seguintes técnicas:

- representação de pontos de uma curva elíptica em coordenadas projetivas que, somado ao uso do modelo de Montgomery, resultaram não só algoritmos mais compactos mas também menor número de inversões calculadas durante as etapas de geração de chaves e do cálculo do segredo compartilhado;

- seleção de primos na forma $p=2^{e_{A}} 3^{e_{B}}-1$, onde $2^{e_{A}}$ e $2^{e_{B}}$ são menores que um múltiplo de 64. A escolha de formato dos primos permitiu alterar o algoritmo de multiplicação de Montgomery para otimizar a aritmética modular, enquanto limitar o tamanho de $2^{e_{A}}$ e $2^{e_{B}}$ torna mais fácil a implementação tanto da aritmética em plataformas com acesso a instruções vetoriais, quanto na multiplicação de Karatsuba com redução deferida;

- escolha dos subgrupos de torção de corpo base e traço zero ao calcular os emparelhamentos no processo de geração de chaves, o que permite realizar as multiplicações iniciais da geração de chaves no corpo base e consequentemente resulta em uma pequena melhoria de tempo e tamanho de chaves. 
Inspirados por essas técnicas, Brian Koziel, Amir Jalali, Reza Azarderakhsh, Mehran Kermani e David Jao apresentaram um implementação otimizada em linguagem C e assembly para a arquitetura ARM, chamada NEON-SIDH (KOZIEL et al., 2016). O trabalho também propôs um conjunto de números primos para níveis de segurança quântica de 85, 128 e 170 bits e analisou o uso de coordenadas afins em substituição às coordenadas projetivas, que provou-se mais eficiente para a arquitetura ARM uma vez que a razão entre inversões e multiplicações possui aproximadamente metade do custo quando comparado à arquitetura x 86 . A implementação obteve operações aritméticas $3 \times$ vezes mais rápidas em relação à biblioteca GMP, e a troca de chaves tornou-se $3 \times$ vezes mais rápida com relação à implementação até então mais rápida para embarcadas (AZARDERAKHSH; FISHBEIN; JAO, 2014) em um dispositivo Cortex-A15 considerando-se o nível de segurança de 85 bits.

Apesar do tamanho de chaves do SIDH ser um dos menores dentre as alternativas pósquânticas até então conhecidas, (AZARDERAKHSH et al., 2016) introduziram uma variante do esquema original com chaves comprimidas, permitindo representar chaves públicas aproximadamente $50 \%$ menores em relação ao trabalho original. Para isso, a variante substitui a representação da curva base $E$ por seu j-invariante e decompõe os pontos $P$ e $Q$ em coeficientes menores em relação a uma base de torção fixa. Contudo, a compressão de chaves apresentada demanda cálculos computacionais tão custosos que a latência da compressão de cada entidade chega a ser até dez vezes maior que uma rodada completa de troca de chaves sem compressão. $\mathrm{O}$ trabalho de (COSTELLO et al., 2017) identificou os principais gargalos inseridos pela técnica de compressão de chaves e apresentou novos algoritmos e melhorias que tornaram o desempenho de uma rodada de troca de chaves aproximadamente $10 \times$ vezes mais rápido em relação ao resultado anterior. As melhorias apresentadas consistem em:

- utilizar-se de resultados que emergem do 2- e 3-descenso das curvas elípticas para evitar multiplicações custosas pelo cofator ao testar a ordem dos pontos gerados. Esses resultados combinados com a codificação do Elligator 2 (BERNSTEIN et al., 2013) para a geração eficiente de pontos em $E$, possibilitam a geração eficiente de bases de $n$-torção;

- aplicar técnicas da literatura de curvas elípticas no cálculo de emparelhamentos, como a substituição do emparelhamento de Weil pelo de Tate, ordenação no cálculo de emparelhamentos para que apenas dois laços de Miller sejam utilizados, além de melhorias nesse algoritmo para que as inversões tenham custo nulo;

- realizar o cálculo do logaritmo discreto no subgrupo $\mu_{n}$ do grupo multiplicativo $G_{P+1} \in \mathbb{F}_{p^{2}}^{*}$ através de uma variante do algoritmo Pohlig-Hellman a qual utiliza-se de janelas para reduzir a complexidade do algoritmo original. O subgrupo em questão permite o cálculo eficiente de quadrados e cubos ciclotômicos, além de inversões que consistem apenas do cálculo do conjugado; 
- redução do tamanho da chave pública através da normalização dos coeficientes de $P$ e $Q$ em relação à base. Com isso, o tamanho passa de aproximadamente $4 \log p$ bits para $\frac{7}{2} \log p$ bits;

- redução no tempo da descompressão de chaves através não só do primeiro passo, mas também pela absorção de multiplicações por escalares durante o cálculo do segredo compartilhado.

O trabalho também discute brevemente técnicas de validação para evitar ataques ativos ao esquema, como ataques de "subgrupos pequenos" ou "curvas inválidas". Entretanto as técnicas apresentadas consideram apenas o caso efêmero da troca de chaves e deixam o caso estático vulnerável. Esses ataques incorrem da utilização de pontos auxiliares ao lidar com as dificuldades da não-comutatividade e já haviam sido abordados também de forma breve durante o Workshop de 2015 organizado pelo NIST (KIRKWOOD et al., 2015). Para preencher a lacuna deixada pelos trabalhos anteriores, (GALBRAITH et al., 2016) descrevem de forma detalhada um ataque à variante estática do protocolo. O ataque é viável mesmo considerando-se as técnicas de validação apresentadas por (COSTELLO et al., 2017), porém torna-se inviável através do método proposto por (KIRKWOOD et al., 2015), que faz uso transformação de Fujisaki-Okamoto e agrega custo computacional considerável.

Em 2017, foi proposto como candidato à competição do NIST um mecanismo de encapsulamento de chaves chamado Supersingular Isogeny Key Encapsulation - SIKE (SIKE, 2017), baseado no esquema de troca de chaves SIDH. O SIKE faz uso da curva inicial é $E_{0} / \mathbb{F}_{p^{2}}: y^{2}=x^{3}+6 x^{2}+x$ e todas as curvas envolvidas estão no modelo de Montgomery com coeficiente $B=1$. Suas chaves são resistentes ao ataque às chaves públicas descrito por (GALBRAITH et al., 2016) e portanto podem ser reutilizadas. A proposta inicial enviada à competição não dava suporte à compressão de chaves, mas graças aos resultados obtidos por trabalhos subsequentes, incluindo os resultados que serão apresentados ao longo deste trabalho, a compressão de chaves se tornou uma opção de uso na implementação padrão.

Em 2018, um novo criptossistema baseado em problemas intratáveis envolvendo isogenias entre curvas elípticas chamado chamado CISDH (Commutative Supersingular Isogeny Diffie-Hellman) foi proposto (CASTRYCK et al., 2018). Esse criptossistema refina o esquema original de Couveignes-Rostovtsev-Stolbunov com o uso de certas curvas supersingulares que aumentam drasticamente sua eficiência (sem se aproximar, porém, da eficiência de SIDH). Apesar do ataque quântico de complexidade subexponencial já mencionado, o esquema não é inviabilizado por conta de sua premissa básica (pela mesma razão que ataques subexponenciais clássicos não inviabilizam o uso de RSA num cenário pré-quântico).

Vale ressaltar aqui que a semelhança de SIDH com CSIDH restringe-se à utilização de curvas supersingulares e à escolha do nome (questionável, por produzir uma impressão errônea de relação mais extensa entre os dois protocolos). Estrutural e algebricamente, os dois 
esquemas são completamente diferentes, e por esta razão uma exploração mais detalhada de CSIDH transcende o escopo desta pesquisa ${ }^{1}$.

1 Por exemplo, uma chave pública CSIDH consiste apenas no coeficiente característico de uma curva de Montgomery, enquanto que uma chave SIDH inclui também a imagem de uma isogenia secreta com núcleo numa das torções sobre uma base da torção complementar, que é onde se aplicam as técnicas de compressão resultantes deste trabalho de pesquisa. 



\section{Fundamentação teórica}

Nesse capítulo descreve-se os conceitos matemáticos que serão utilizados como blocos de construção para compreender o funcionamento da troca de chaves com isogenias supersingulares.

\subsection{Estruturas algébricas}

Um monoide é constituído por um conjunto de elementos e uma operação associativa que, dados dois elementos do conjunto, resulta em um elemento do conjunto. Esse conjunto deve possuir o elemento neutro ou identidade, onde todo elemento operado com o elemento neutro resulta no próprio elemento.

Definição 1 (Monoide). Um conjunto de elementos $\mathbb{M}$ com um operador binário o é dito um monoide $(\mathbb{M}, \circ)$ se segue as seguintes propriedades:

1. Fecho: $a \circ b=c \mid a, b, c \in \mathbb{M}$.

2. Associatividade: $(a \circ b) \circ c=a \circ(c \circ b) \mid a, b, c \in \mathbb{M}$.

3. Elemento neutro: $a \circ e=a, \forall a \in \mathbb{M}$

Um grupo é um monoide com uma propriedade adicional: existe também um elemento inverso, onde um elemento operado com seu inverso resulta no elemento neutro.

Definição 2 (Grupo). Um conjunto de elementos $\mathbb{G}$ com um operador binário o é dito um grupo $(\mathbb{G}, \circ)$ se segue as seguintes propriedades:

1. ( $\mathbb{G}, \circ)$ é um monoide.

2. Elemento inverso: $\forall a \in \mathbb{G}, \exists a^{\prime} \in \mathbb{G} \mid a \circ a^{\prime}=e$.

Exemplos:

- os números inteiros $\mathbb{Z}$ formam um grupo com a operação de adição usual;

- os números inteiros $\mathbb{Z}$ não formam um grupo com a operação de multiplicação usual $(\mathbb{Z},+$ ) pois não existe inverso para todos elementos: $2 \cdot \frac{1}{2}=1$, porém $\frac{1}{2} \notin \mathbb{Z}$.

Alguns grupos destacam-se pelas características que apresentam. Define-se aqui grupos abelianos e de torção. 


\begin{tabular}{|c|c|c|c|c|}
\hline Notação & Operador & Elem. neutro & Elem. inverso & $m$ operações \\
\hline Aditiva & + & 0 & -1 & $m \cdot a$ \\
\hline Multiplicativa & $\cdot$ & 1 & $a^{-1}$ & $a^{m}$ \\
\hline
\end{tabular}

Tabela 1 - Tipos de notações: aditiva e multiplicativa

Definição 3 (Grupo abeliano). Um conjunto de elementos $\mathbb{G}$ com um operador binário o é dito um grupo abeliano $(\mathbb{G}, \circ)$ se segue as seguintes propriedades:

\section{1. (G, o) é um grupo.}

2. Comutatividade: $a \circ b=b \circ a, \forall a, b \in \mathbb{G}$.

Exemplos:

- o conjunto de matrizes inversíveis $n \times n$ forma um grupo abeliano sob a operação de adição de matrizes;

- o conjunto de matrizes inversíveis $n \times n$ não forma um grupo abeliano sob a operação de multiplicação de matrizes por ser não-comutativa.

Apesar de existirem diferentes operações binárias, é comum na literatura atribuir os operadores $(+)$ e $(\cdot)$ a grupos aditivos e multiplicativos, respectivamente. Contudo, essas operações não necessariamente correspondem às operações de adição ou multiplicação usuais. $\mathrm{Na}$ multiplicação de matrizes $n \times n$, por exemplo, cada elemento $c_{i, j}$ da matriz resultante é dado por $\sum_{k=1}^{n} a_{i, k} \cdot b_{k, j}$, diferente da multiplicação usual entre dois elementos.

O uso de grupos aditivos e multiplicativos na literatura dá origem a dois tipos de notação que será usado daqui em diante:

Dado que é possível adicionar um elemento $a$ de um grupo $\mathbb{G}$ a si mesmo um número arbitrário $m>0$ de vezes, é natural definir a multiplicação escalar $m \cdot a \equiv a+a+\cdots+a$ ( $m$ termos). Por extensão, define-se também [0] $a \equiv 0$, e para coeficientes negativos $-m \cdot a \equiv m \cdot-a$. Em notação multiplicativa, a mesma operação é chamada exponenciação, escrevendo-se $a^{m} \equiv a \cdot a \cdots \cdots a$ ( $m$ fatores), $a^{0} \equiv 1$, e $a^{-m} \equiv\left(a^{-1}\right)^{m}$. Dado o elemento $b=m \cdot a$ (ou $b=a^{m}$ ), o fator (ou expoente) $m$ é chamado índice (ou logaritmo discreto) de $b$ com relação à base $a$.

Para definir um grupo de torção, é necessário definir a ordem de um elemento do grupo, que pode ser confundida com a ordem de um grupo. Apesar de nomes e notações similares, a ordem do grupo representa o número de elementos do conjunto, enquanto a ordem de um elemento representa o menor número de vezes que um elemento deve ser operado consigo mesmo até resultar no elemento neutro. 
Definição 4 (Ordem de um grupo). A ordem ou cardinalidade de um grupo $\mathbb{G},|\mathbb{G}|$, é o número de elementos do conjunto. Se o número de elementos é finito, o grupo é dito finito. Caso contrário o grupo é dito infinito.

Definição 5 (Ordem de um elemento do grupo). A ordem de um elemento a $\in \mathbb{G},|a|$, é o menor valor $m \in \mathbb{N}^{*}$ tal que $|a|=a^{m}=1$ em notação multiplicativa ou $|a|=m \cdot a=0$ em notação aditiva. Caso $\nexists$ m, sua ordem é dita infinita.

Definição 6 (Grupo de torção). Um grupo de torção ou periódico é um grupo em que todos seus elementos possuem ordem finita.

Definição 7 (Grupo cíclico). Um grupo $\mathbb{G}$ é dito cíclico se é gerado por um único elemento $g \in \mathbb{G}$, chamado de gerador do grupo. Em notação aditiva, $\forall a \in \mathbb{G}, \exists k \in \mathbb{Z}^{+} \mid k a=g$ ou $\forall a \in \mathbb{G}, \exists k \in \mathbb{Z}^{+} \mid a^{k}=g$ em notação multiplicativa

Definição 8 (Resíduo quadrático). Se existe um inteiro $x, 0<x<p$, que satisfaz a congruência:

$$
x^{2}=q(\bmod p)
$$

então q é dito um resíduo quadrático $(\bmod p)$.

Um anel é formado por um conjunto de elementos e dois operadores binários os quais serão chamados de adição e multiplicação por conveniência.

Definição 9 (Anel). Um conjunto de elementos $\mathbb{R}$ com dois operadores binários $+e \cdot e$ é dito um anel $(\mathbb{R},+, \cdot)$ se:

1. $(\mathbb{R},+)$ é um grupo abeliano.

2. $(\mathbb{R}, \cdot)$ é um monoide.

3. Distributividade de $\cdot \operatorname{sob}+$ :

- à esquerda: $a \cdot(b+c)=a \cdot b+a \cdot b, \forall a, b, c \in \mathbb{R}$

- à direita: $(b+c) \cdot a=b \cdot a+c \cdot a, \forall a, b, c \in \mathbb{R}$

Exemplos:

- os números inteiros $\mathbb{Z}$ formam um anel com as operações usuais de adição e multiplicação;

- o conjunto de matrizes $n \times n$ com coeficientes reais forma um anel com as operações de adição e multiplicação de matrizes; 
Considera-se agora estruturas algébricas mais complexas: os corpos e extensões de corpos.

Um corpo é um anel comutativo com unidade em que todo elemento diferente de 0 possui um elemento inverso com relação à multiplicação.

Definição 10 (Corpo). Um conjunto de elementos $\mathbb{F}$ com dois operadores binários $+e \cdot e$ é dito um corpo $(\mathbb{F},+, \cdot)$ se:

\section{1. $(\mathbb{F},+)$ é um grupo abeliano.}

2. $(\mathbb{F} \backslash\{0\}, \cdot)$, onde 0 é o elemento neutro aditivo, é um grupo abeliano.

3. Distributividade de $\cdot$ sob $+\grave{a}$ direita e à esquerda.

Exemplos:

- Os números racionais $\mathbb{Q}$, reais $\mathbb{R}$ e complexos $\mathbb{C}$ formam um corpo com as operações usuais de adição e multiplicação.

- Os números inteiros módulo $p$ (isto é, $\mathbb{Z} / p \mathbb{Z}$ ) formam um corpo se, e somente se, $p$ for um número primo.

Uma propriedade interessante de um corpo é sua característica, que é o número de vezes que a identidade multiplicativa de $\mathbb{F}$ deve ser adicionada a si mesma para que o resultado seja igual à identidade aditiva.

Definição 11 (Característica de um corpo). A característica de um corpo $\mathbb{F}$, char $(\mathbb{F})$, é o menor valor $m \in \mathbb{N}^{*}$ tal que char $(\mathbb{F})=m \cdot 1=0$. Caso $\nexists m$, define-se char $(\mathbb{F})=0$.

Uma extensão de corpo também um corpo que, como o próprio nome sugere, amplia o um corpo chamado corpo base adicionando a ele novos elementos.

Definição 12 (Extensão de um corpo). Um corpo $\mathbb{F}_{e}$ é dito ser uma extensão de um corpo $\mathbb{F}$, denotado por $\mathbb{F}_{e} / \mathbb{F}$, se $\mathbb{F} \subset \mathbb{F}_{e}$.

Se $\mathbb{F}$ é um subcorpo de um corpo maior $\mathbb{F}^{\prime}$, então é possível criar uma extensão de $\mathbb{F}$ escolhendo elementos $a \in \mathbb{F}_{e}$, mas $a \notin \mathbb{F}$ de tal forma que $\mathbb{F}(a)$ seja o menor subcorpo de $\mathbb{F}^{\prime}$ contendo $\mathbb{F}$ e $a$. Como principal exemplo tem-se os números complexos que formam uma extensão dos números reais através da adição do número imaginário $i=\sqrt{-1}$.

Por conveniência, definem-se aqui também as noções de grupo de classe, álgebra de quatérnions e nais polinomiais, que serão úteis no contexto específico de certos problemas computacionais intratáveis envolvendo isogenias sobre curvas supersingulares. 
Definição 13 (Grupo de classe). $O$ grupo de classe de um corpo numérico algébrico $K$ é o grupo quociente $J_{K} / P_{K}$ entre o grupo $J_{K}$ de ideais fracionários do anel de inteiros de $K$ e o subgrupo $P_{K}$ dos ideais principais de $J_{K}$.

Definição 14 (Álgebra de quatérnions). Dado um corpo finito $\mathbb{F}_{q}$ de característica ímpar, uma álgebra de quatérnions sobre $\mathbb{F}_{q}$ é um espaço vetorial de dimensão 4 sobre $\mathbb{F}_{q}$ definido por uma base $(1, i, j, k)$ e munido de uma operação de multiplicação especificada pelas regras $i^{2}=u$, $j^{2}=v, i j=-j i=k$ onde $u, v \in \mathbb{F}_{q} \backslash\{0\}$ são elementos fixos para cada álgebra.

Definição 15 (Anel polinomial). Um anel polinomial $\mathbb{R}[x]$ sobre $x \in \mathbb{F}$, onde $\mathbb{F}$ é um corpo, é o conjunto de polinômios em x na forma:

$$
p(x)=a_{0}+a_{1} x+a_{2} x^{2}+\cdots+a_{n} x^{n}
$$

onde os coeficiente $a_{0}, a_{1}, \cdots, a_{n} \in \mathbb{R}$.

\subsection{Curvas elípticas}

Definição 16 (Curva elíptica). Uma curva elíptica $E$ é o conjunto das soluções $[X: Y: Z] \neq$ [0 : $0: 0]$ de uma equação da forma $P(X, Y, Z)=0$ para algum polinômio homogêneo $P$ em 3 variáveis, particionadas em classes de equivalência pela relação projetiva $[\lambda X: \lambda Y: \lambda Z] \sim[X:$ $Y: Z], \forall \lambda \neq 0$. Um ponto da curva E é uma das classes de equivalência estabelecidas por essa relação.

Definição 17 (Ponto da curva). Um ponto da curva E é uma das classes de equivalência estabelecidas pela relação projetiva. O número de pontos de uma curva elíptica E é chamado ordem da curva, denotada \#E.

Em particular, $[X: Y: Z] \sim[X / Z: Y / Z: 1]$ para $Z \neq 0,[X: Y: 0] \sim[X / Y: 1: 0]$ para $Y \neq 0$, e $[X: 0: 0] \sim[1: 0: 0]$ para $X \neq 0$. As soluções com $Z \neq 0$ são comumente chamadas pontos finitos da curva. Isso permite definir uma equação afim para uma dada curva elíptica, a saber, a curva consiste soluções $(x, y)$ da equação $p(x, y)=0$ para $p(x, y):=P(x, y, 1)$, juntamente com pontos adicionais associados às soluções projetivas $\operatorname{com} Z=0$.

O número de pontos de uma curva elíptica sobre um corpo finito é sempre um valor próximo ao número de elementos desse corpo. Este resultado é conhecido como teorema de Hasse (SILVERMAN, 1986, Capítulo V, Seção 1, Teorema 1.1):

Teorema 18. A ordem $|E|$ de uma curva elíptica $E$ sobre o corpo $\mathbb{F}_{q}$ satisfaz as relações:

$$
q+1-2 \sqrt{q} \leqslant|E| \leqslant q+1+2 \sqrt{q} .
$$

Embora um ponto de uma curva elíptica em forma afim consista em um par $(x, y)$ de elementos do corpo subjacente, é possível representar um ponto de maneira mais compacta 
aproveitando a equação da curva. A equação $p(x, y)=0$ tem a propriedade adicional que, para um dado valor de $x$, existem no máximo $\operatorname{deg}_{y}(p)$ valores de $y$ que a satisfazem, e vice-versa. Via de regra, $\operatorname{deg}_{y}(p)=2$, e com isso basta um único bit além do valor da abscissa $x$ para distinguir entre os valores da ordenada $y$ que tornam o ponto $(x, y)$ uma solução da equação $p(x, y)=0$. Essa representação de um ponto $(x, y)$ como um par $(x, \operatorname{lsb}(y))$ (onde $\operatorname{lsb}(y)$ designa o bit menos significativo de $y$, denomina-se representação comprimida do ponto.

\subsubsection{Modelo de Weierstrass}

O modelo mais amplamente adotado para descrever curvas elípticas independentemente de aplicação é o chamado modelo de Weierstrass.

Definição 19 (Curva de Weierstrass). Seja $\mathbb{F}$ um corpo e $a, b, c, d, e \in \mathbb{F}$. Uma curva elíptica no modelo de Weierstrass sobre $\mathbb{F}$, denotada $E_{W}(\mathbb{F})$, é o conjunto de pares $(x, y) \in \mathbb{F} \times \mathbb{F}$ que satisfazem uma equação da forma

$$
y^{2}+a x y+b y=x^{3}+c x^{2}+d x+e
$$

a que se acrescenta por conveniência um ponto extra, $O$, chamado ponto no infinito.

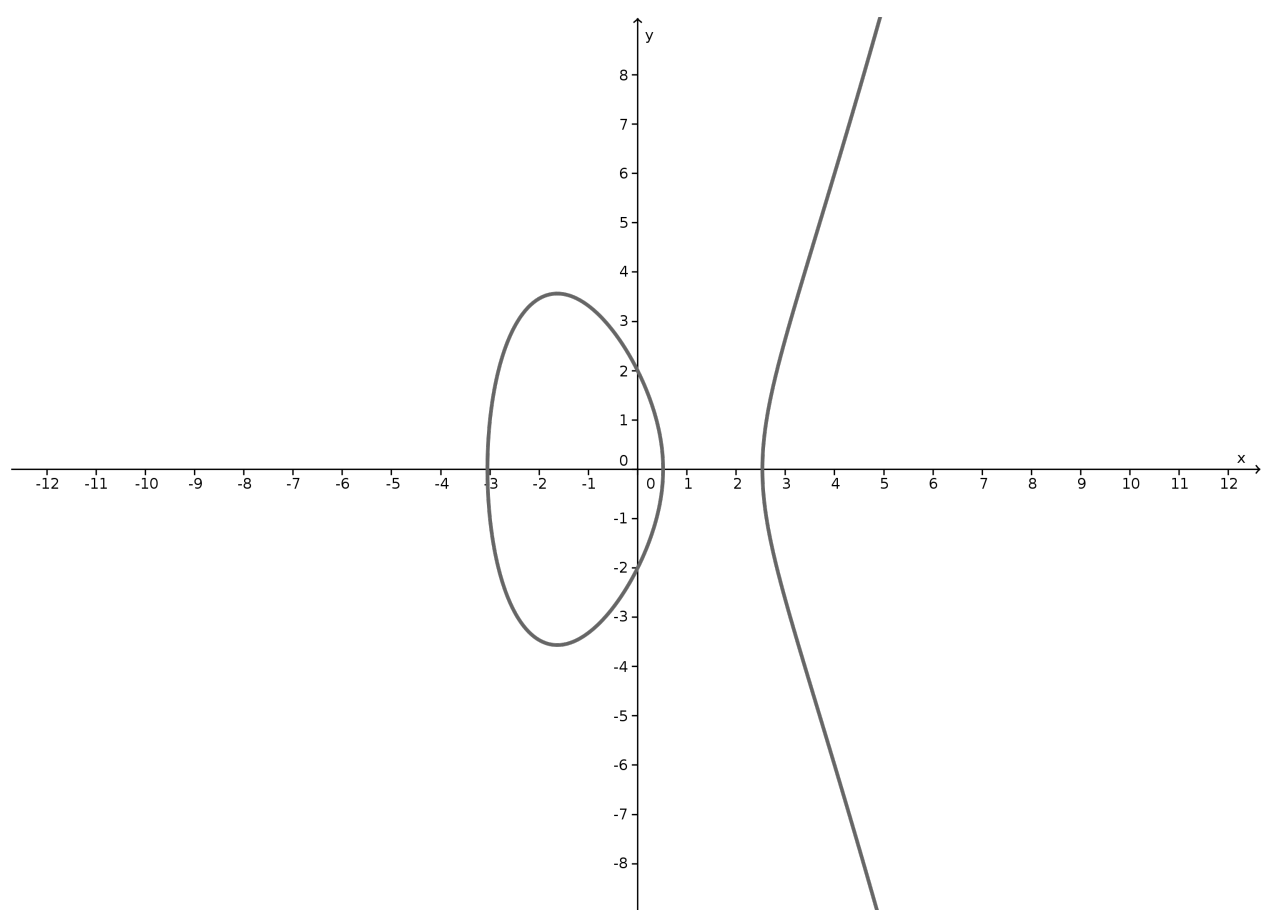

Figura 1 - Curva de Weierstrass $y^{2}=x^{3}-8 x+4$ sobre $\mathbb{R}$.

Fonte: Barreto (1999).

A equação 3.1 pode ser simplificada por meio de mudanças de coordenadas. Se $\operatorname{char}(\mathbb{F})=$ 
2, a equação reduz-se a uma das formas:

$$
\begin{aligned}
& y^{2}+x y=x^{3}+a x^{2}+b \\
& y^{2}+c y=x^{3}+a x+b
\end{aligned}
$$

Se $\operatorname{char}(\mathbb{F})=3$, a equação 3.1 pode ser reescrita:

$$
\begin{aligned}
& y^{2}=x^{3}+a x^{2}+b \\
& y^{2}=x^{3}+a x+b
\end{aligned}
$$

Finalmente, $\operatorname{char}(\mathbb{F}) \neq 2,3$ a equação 3.1 pode ser transformada em:

$$
y^{2}=x^{3}+a x+b
$$

O nome "curva" aqui é um abuso de linguagem. Embora o gráfico de uma equação do tipo 3.6 sobre $\mathbb{R}$ tenha realmente o aspecto de uma curva (Figura 1), a mesma equação sobre outro corpo pode determinar um conjunto discreto de pontos (Figura 2). Dado um ponto $P=\left(x_{P}, y_{P}\right)$ de uma curva, utiliza-se a notação $\operatorname{xcoord}(P) \equiv x_{P}, \operatorname{xcoord}(P) \equiv y_{P}$ para as coordenadas do ponto $\mathrm{P}$.

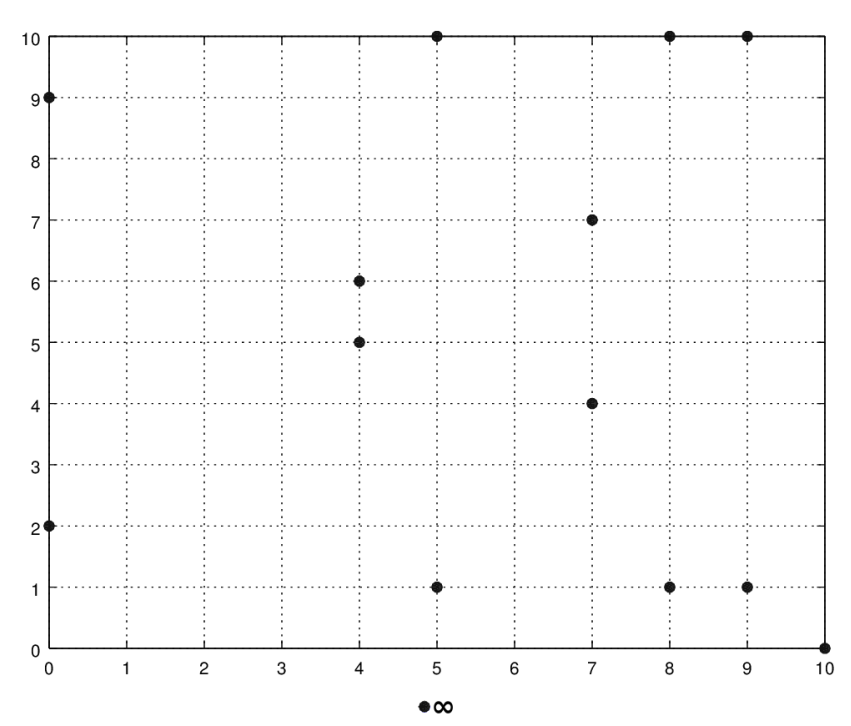

Figura 2 - Curva $y^{2}=x^{3}-8 x+4$ sobre $\mathbb{F}_{11}$.

Fonte: Barreto (1999).

\subsubsection{Lei de grupo de Weierstrass (secantes e tangentes)}

É possível introduzir uma estrutura de grupo sobre os pontos de determinadas curvas elípticas. Para isso, é preciso definir uma operação binária apropriada entre esses pontos, e 
também especificar a identidade e o elemento inverso de um ponto qualquer com relação a essa operação binária.

Por simplicidade, apresenta-se a lei de grupo no modelo de Weierstrass. A construção da lei de grupo para curvas sobre $\mathbb{R}$ tem caráter geométrico. Uma tal curva é simétrica em relação ao eixo $x$. A identidade é definida como sendo o ponto no infinito $(O)$, o inverso de um ponto $P=(x, y)$ é definido como sendo o ponto $-P=(x,-y)$ (isto é, o ponto simétrico a $P$ com relação ao eixo $x$, Figura 3 ), e a soma de dois pontos $P$ e $Q$ é definida no Algoritmo 1.

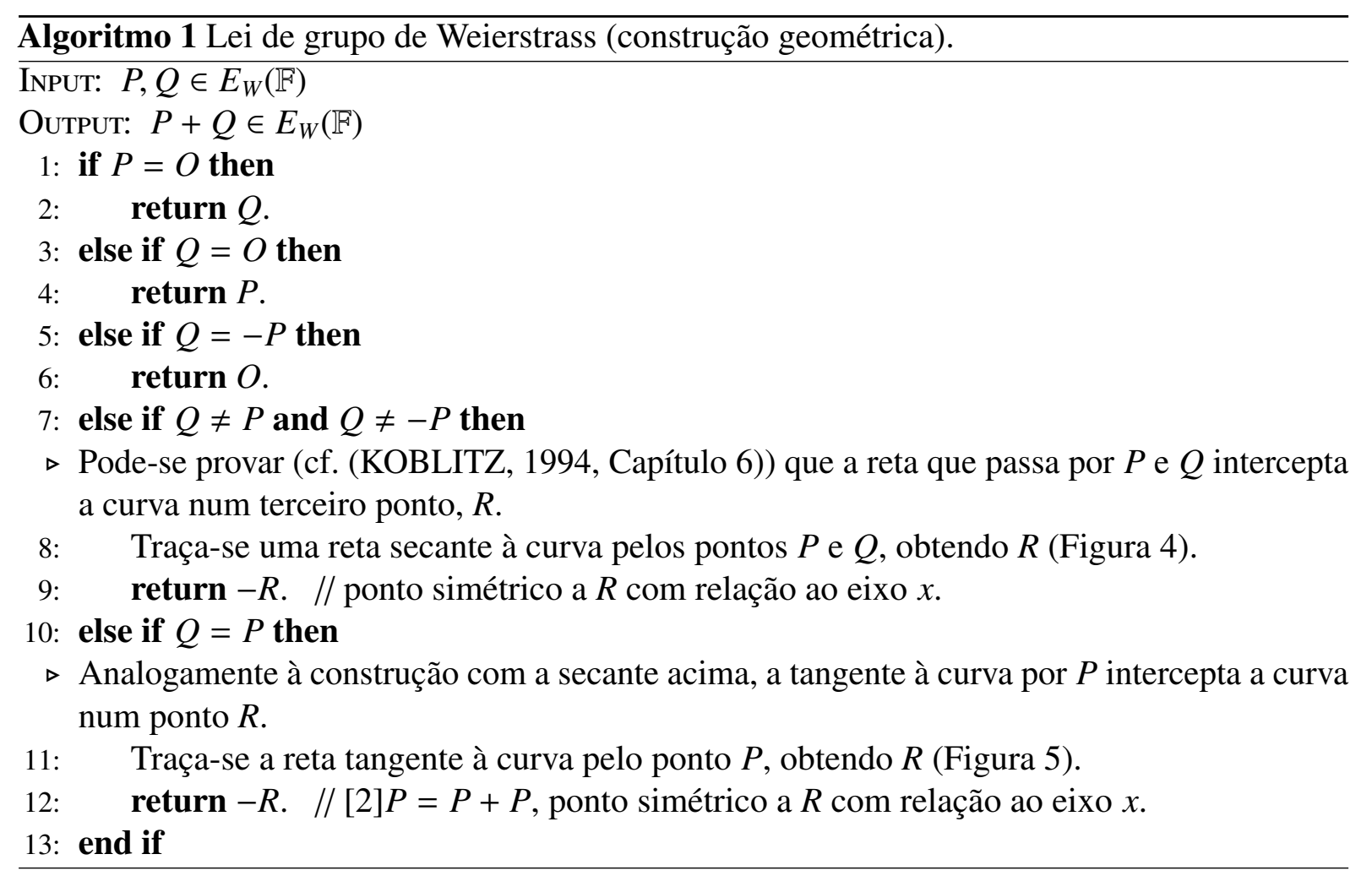

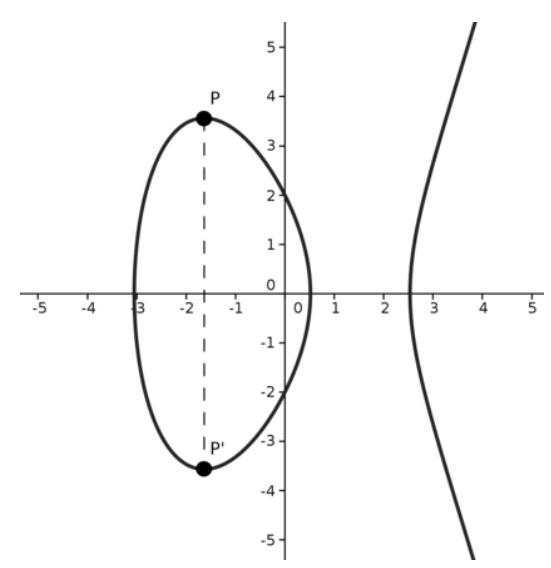

Figura 3 - Inverso de um ponto da curva.

Fonte: Barreto (1999).

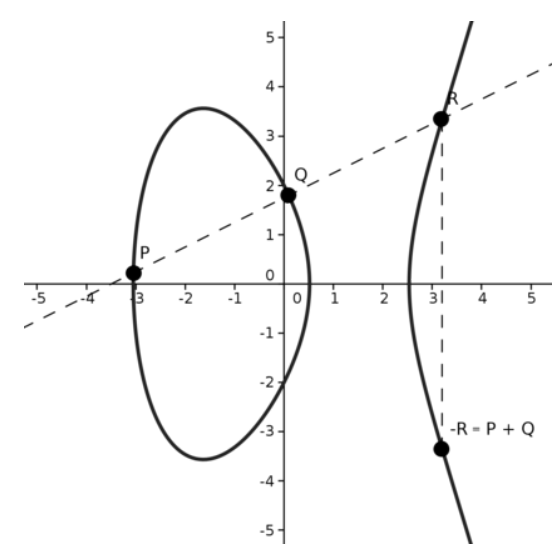

Figura 4 - Soma de pontos (regra da secante). Fonte: Barreto (1999). 


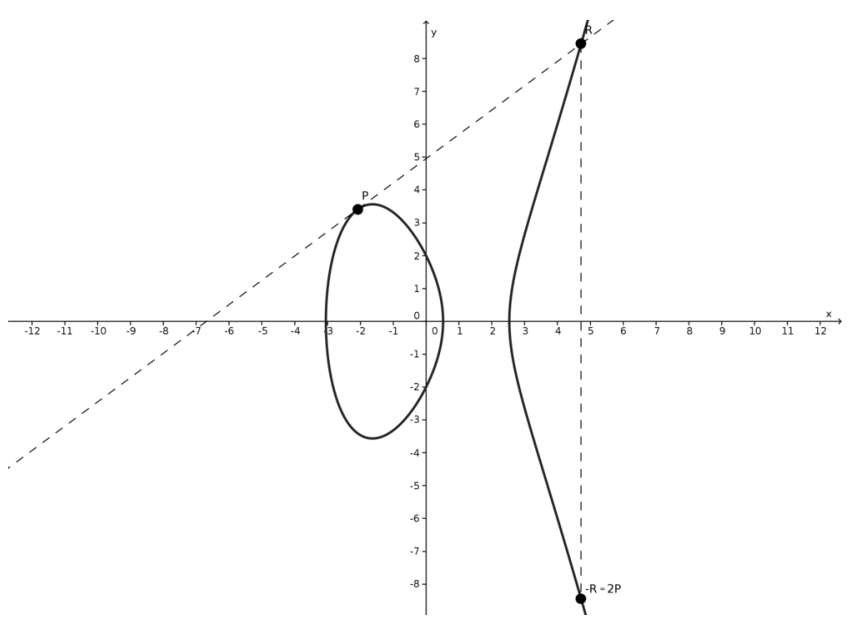

Figura 5 - Duplicação de um ponto (regra da tangente).

Fonte: Barreto (1999).

Estranha como essa construção possa parecer, ela define uma lei de grupo sobre os pontos da curva, desde que seja possível traçar a reta tangente a um ponto qualquer da curva. Esta condição é satisfeita se, e somente se, o polinômio $f(x):=x^{3}+a x+b$ não tiver raízes múltiplas, isto é, se o discriminante da curva, $\Delta:=-16\left(4 a^{3}+27 b^{2}\right)$, for diferente de zero.

A forma do discriminante para cada tipo de curva é dada na Tabela 2 abaixo.

\begin{tabular}{|c|c|}
\hline tipo da curva & discriminante \\
\hline equação 3.2 & $b$ \\
equação 3.3 & $c^{4}$ \\
equação 3.4 & $a^{2}\left(b^{2}-a c\right)-b^{3}$ \\
equação 3.5 & $a^{2}\left(b^{2}-a c\right)-b^{3}$ \\
equação 3.6 & $-16\left(4 a^{3}+27 b^{2}\right)$ \\
\hline
\end{tabular}

Tabela 2 - Discriminante de uma curva elíptica

Essa observação fornece a equação geral das curvas elípticas (no modelo de Weierstrass) sobre $\mathbb{R}$ para as quais não se pode definir uma lei de grupo, chamadas curvas singulares ou degeneradas: $y^{2}=x^{3}-3 t^{2} x+2 t^{3}$, cujas raízes são $t$ e $-2 t$ onde $t$ é raiz dupla se diferente de zero (Figuras 6 e 7) e raiz tripla caso contrário (Figura 8). 

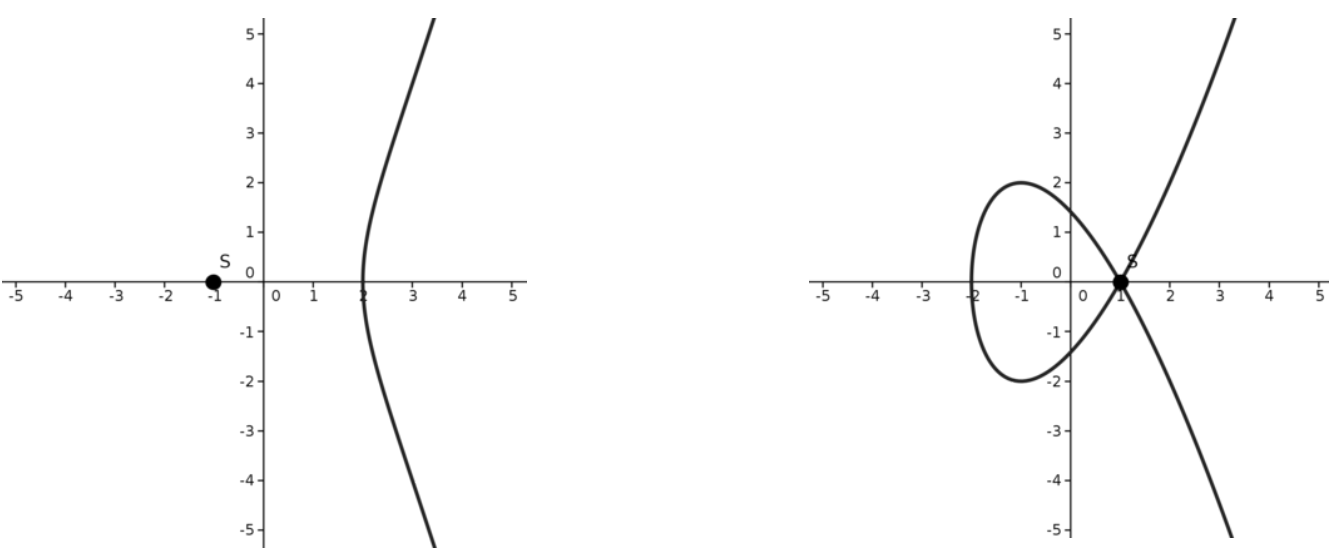

Figura 6 - Curva degenerada $y^{2}=x^{3}-3 x-2$, Figura 7 - Curva degenerada $y^{2}=x^{3}-3 x+2$, singular em $S=(-1,0)$. singular em $S=(1,0)$.

Fonte: Barreto (1999).

Fonte: Barreto (1999).

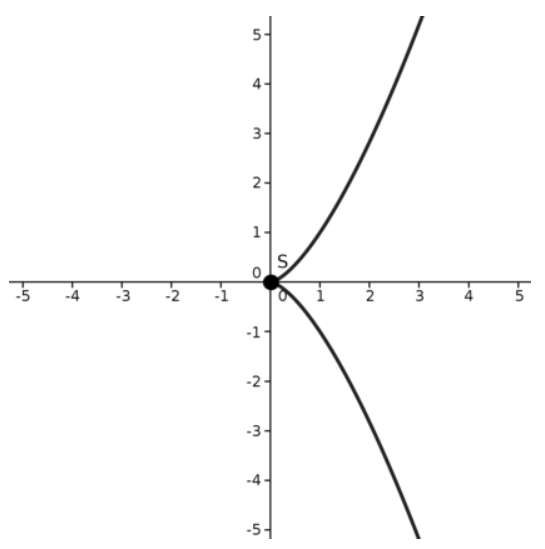

Figura 8 - Curva degenerada $y^{2}=x^{3}$, singular em $S=(0,0)$.

Fonte: Barreto (1999).

A partir da construção geométrica, deduzem-se as expressões algébricas para o cálculo das coordenadas do ponto $S=\left(x_{S}, y_{S}\right)=P+Q$ a partir das coordenadas de $P=\left(x_{P}, y_{P}\right)$ e $Q=\left(x_{Q}, y_{Q}\right)$.

$$
\begin{aligned}
x_{S} & =\lambda^{2}-x_{P}-x_{Q}, \\
y_{S} & =\lambda\left(x_{P}-x_{S}\right)-y_{P}, \\
\lambda & = \begin{cases}\frac{y_{Q}-y_{P}}{x_{Q}-x_{P}}, & \text { se } P \neq Q, \\
\frac{3 x_{P}^{2}+a}{2 y_{P}}, & \text { se } P=Q .\end{cases}
\end{aligned}
$$

Estas expressões induzem uma lei de grupo em curvas sobre qualquer corpo com característica diferente de 2 e de 3 com discriminante não nulo, mesmo não se podendo falar de construções geométricas por secantes e tangentes para esses corpos. 
Expressões análogas existem para definir a lei de grupo em curvas sobre corpos de característica 2 ou 3. Por exemplo, para curvas satisfazendo a equação 2 a lei de grupo é definida pelas seguintes regras:

$$
\begin{aligned}
x_{S} & =\lambda^{2}+\lambda+x_{P}+x_{Q}+a, \\
y_{S} & =\lambda\left(x_{P}+x_{S}\right)+x_{S}+y_{P}, \\
\lambda & = \begin{cases}\frac{y_{Q}-y_{P}}{x_{Q}-x_{P}}, & \text { se } P \neq Q, \\
x_{P}+\frac{y_{P}}{x_{P}}, & \text { se } P=Q .\end{cases}
\end{aligned}
$$

Os grupos elípticos, como quaisquer outros grupos, podem ser cíclicos. Neste caso, existe um ponto cujos múltiplos varrem toda a curva (Figura 9, onde o ponto no infinito $O$ é mostrado por clareza).

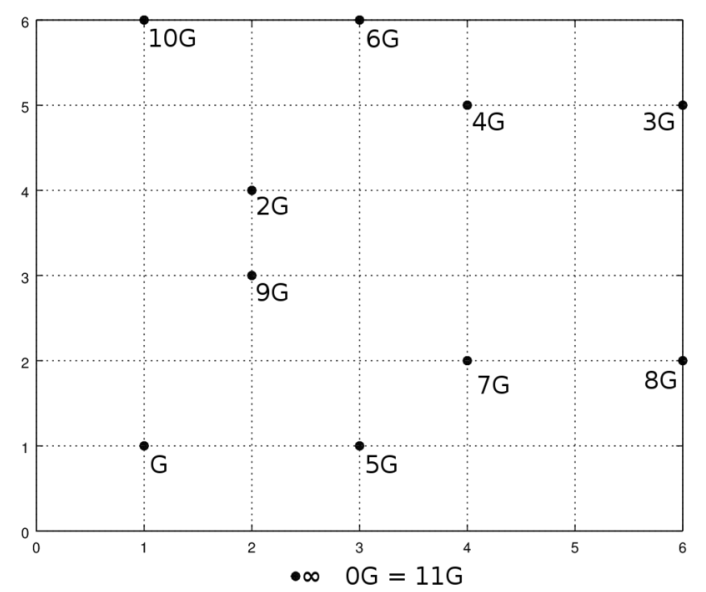

Figura 9 - Curva $y^{2}=x^{3}+x-1$ sobre $\mathbb{F}_{7}$ gerada por $G=(1,1)$.

Fonte: Barreto (1999).

Uma das vantagens de trabalhar com um gerador é a facilidade de calcular a soma de pontos. Com efeito, dado um gerador $G$ de uma curva de ordem $n$, a soma dos pontos $P=\left[k_{P}\right] G$ e $Q=\left[k_{Q}\right] G$ é $P+Q=\left[k_{S}\right] G$ onde $k_{S}=\left(k_{P}+k_{Q}\right) \bmod n$.

Para aplicações criptográficas, consideram-se curvas que contenham subgrupos cíclicos de ordem prima (o motivo desta restrição é evitar certos tipos de ataque, por exemplo o método Pohlig-Hellman. O melhor caso ocorre quando a própria ordem da curva é prima, mas nem sempre é possível obter essa condição.

Há outros aspectos de segurança de uma curva relacionados com a ordem da curva. Em particular, define-se:

Definição 20 (Curva elíptica supersingular). Uma curva elíptica E sobre um corpo finito $\mathbb{F}_{q}$ de característica pé chamada supersingular se, e somente se, $p \mid t$ onde \#E $=q+1-t$. 
É fácil observar que o número de curvas supersingulares é menor em relação ao número total de curvas possíveis sobre o mesmo corpo finito. Isto decorre do Teorema de Hasse 18, e é concisamente expresso no seguinte corolário (MENEZES, 1993, Corolário 2.11):

Corolário 21. Seja E uma curva definida sobre $F_{q}$. Então E é supersingular se, e somente se, $t^{2} \in\{0, p, 2 p, 3 p, 4 p\}$, onde $t=p+1-|E|$.

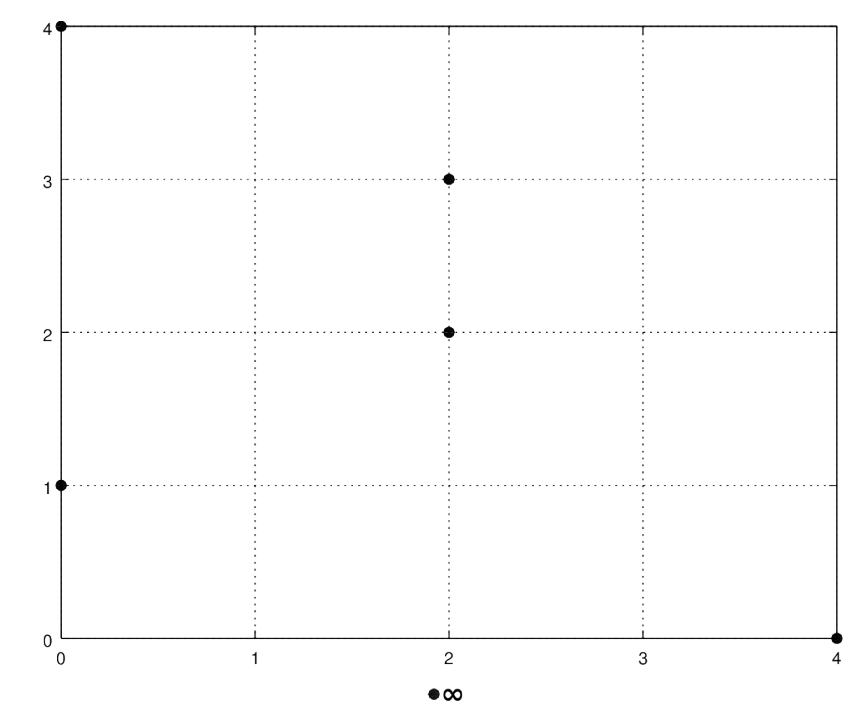

Figura 10 - Curva supersingular $y^{2}=x^{3}+1$ sobre $\mathbb{F}_{5}$.

Fonte: Barreto (1999).

Apesar do uso de curvas supersingulares apresentar um brecha de segurança no contexto de curvas elípticas, o mesmo não ocorre quando o mesmo tipo de curva é empregado no contexto de isogenias, conforme será visto mais adiante.

\subsubsection{Modelo de Montgomery}

Curvas de Montgomery (MONTGOMERY, 1987) foram introduzidas originalmente para acelerar as tarefas de demonstração de primalidade ou de fatoração inteira por meio de curvas elípticas. Como benefício adicional, as mesmas propriedades que tornam as curvas de Montgomery particularmente adequadas àquelas tarefas também constituem vantagens para a implementação de certos tipos de criptossistemas.

Definição 22 (Curva de Montgomery). Seja $\mathbb{F}$ um corpo e $a, b \in \mathbb{F}$. Uma curva elíptica no modelo de Montgomery sobre $\mathbb{F}$, denotada $E_{M}(\mathbb{F})$, é o conjunto de pares $(x, y) \in \mathbb{F} \times \mathbb{F}$ que satisfazem uma equação da forma

$$
B y^{2}=x^{3}+A x^{2}+x,
$$

a que se acrescenta por conveniência um ponto extra, $O$, chamado ponto no infinito. 
Equivalentemente, uma curva de Montgomery admite uma equação da forma $y^{2}=$ $x\left(x^{2}+A x+B\right)$, como se pode concluir por uma mudança simples de coordenadas.

O número de pontos de uma curva de Montgomery é sempre um múltiplo de 4. Para fins criptográficos, escolhe-se sempre uma curva de ordem $4 n$ onde $n$ é um número primo.

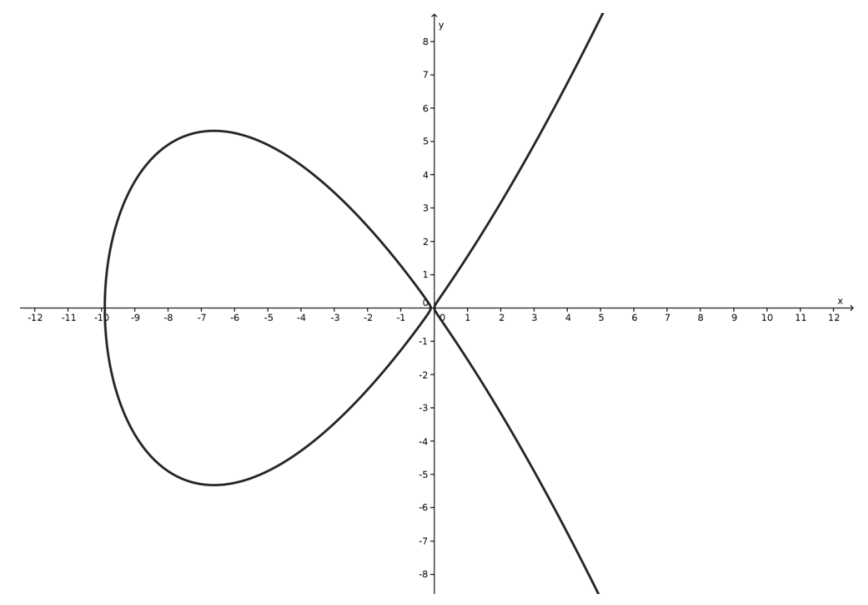

Figura 11 - Curva de Montgomery $5 y^{2}=x^{3}+10 x^{2}+x$.

Fonte: Barreto (1999).

Curvas de Montgomery prestam-se excepcionalmente bem à implementação de protocolos onde apenas multiplicações simples por escalar são necessárias, uma vez que a abscissa do ponto resultante depende apenas da abscissa do ponto original e do fator escalar. Além disso, esse cálculo pode ser feito de maneira essencialmente independente do valor binário detalhado do fator escalar, protegendo essa informação de vários tipos de ataque por canal colateral.

Para facilidade de referência, o Apêndice A traz fórmulas detalhadas para a aritmética em curvas de Montgomery com coordenadas projetivas (que mantêm apenas as coordenas $X$ e $Z)$.

\subsubsection{O $j$-invariante}

A noção de $j$-invariante visa a caracterizar curvas elípticas de maneira independente de meras mudanças de variáveis que conservam a forma da equação da curva.

Especificamente, dada uma equação de curva no modelo de Weierstrass $y^{3}=x^{3}+a x+b$ e um elemento não nulo $\mu$ do corpo finito, a equação $y^{\prime 3}=x^{\prime 3}+a^{\prime} x^{\prime}+b^{\prime} \operatorname{com} x^{\prime}:=\mu^{2} x$, $y^{\prime}:=\mu^{3} y, a^{\prime}:=\mu^{4} a, b^{\prime}:=\mu^{6} b$, define essencialmente a mesma curva em outras coordenadas (mais precisamente, uma curva birracionalmente equivalente). $\mathrm{O} j$-invariante é então definido como uma função dos coeficientes da equação da curva que se mantém inalterado com essa mudança de coordenadas. 
Em forma explícita, o $j$-invariante é definido (WASHINGTON, 2008, Seção 2.7) em função dos coeficientes $a$ e $b$ do modelo de Weierstrass como:

$$
j:=1728 \cdot \frac{4 a^{3}}{4 a^{3}+27 b^{2}} .
$$

No modelo de Montgomery $y^{2}=x^{3}+A x^{2}+x$, o $j$-invariante é dado por:

$$
j:=256 \cdot \frac{\left(A^{2}-3\right)^{3}}{A^{2}-4}
$$

\subsubsection{Emparelhamentos bilineares}

Sistemas criptográficos designados genericamente como "baseados no logaritmo discreto" fundamentam-se em diversos problemas matemáticos distintos, mas estreitamente relacionados, definidos num grupo $\mathbb{G}$. São eles:

- Problema Diffie-Hellman Computacional (CDHP) Dados $P,[a] P,[b] P \in \mathbb{G}$, calcular $[a b] P$.

- Problema Diffie-Hellman Decisional (DDHP) Dados $P,[a] P,[b] P,[c] P \in \mathbb{G}$ onde a ordem de $P$ é $r$, decidir se $c \equiv a b(\bmod r)$.

Obviamente, se o DLP é tratável, então o CDHP também é tratável, e se o CDHP é tratável, então o DDHP também é tratável.

Existem grupos onde o DDHP pode ser eficientemente resolvido. Este é o caso de grupos onde se pode definir a noção de emparelhamento:

Definição 23. Sejam $\mathbb{G}_{1} e \mathbb{G}_{2}$ grupos escritos aditivamente, $\mathbb{G}_{3}$ um grupo escrito multiplicativamente. Um emparelhamento é uma função bilinear, não degenerada, efetivamente computável $e: \mathbb{G}_{1} \times \mathbb{G}_{2} \rightarrow \mathbb{G}_{3}$. Em outras palavras, as seguintes propriedades são válidas para a função e:

- (Bilinearidade) $e\left(P_{1}+P_{2}, Q\right)=e\left(P_{1}, Q\right) \cdot e\left(P_{2}, Q\right) e e\left(P, Q_{1}+Q_{2}\right)=e\left(P, Q_{1}\right) \cdot e\left(P, Q_{2}\right)$ para todo $P, P_{1}, P_{2} \in \mathbb{G}_{1}$ e todo $Q, Q_{1}, Q_{2} \in \mathbb{G}_{2}$. Segue daí que $e([a] P,[b] Q)=e(P, Q)^{a b}$ para todo $a, b \in \mathbb{Z}$.

- (Não-degeneração) Se $e(P, Q)=1$ para todo $Q \in \mathbb{G}_{2}$, então $P=O$. Alternativamente, para todo $P \neq O$ existe $Q \in \mathbb{G}_{2}$ tal que e $(P, Q) \neq 1$.

- (Computabilidade) $O$ cálculo de e $(P, Q)$ é computacionalmente tratável (tempo polinomial).

Suponha que exista um emparelhamento $e: \mathbb{G} \times \mathbb{G} \rightarrow \mathbb{G}^{\prime}$. Nesse caso, dados $P,[a] P$, $[b] P,[c] P \in \mathbb{G}$ onde a ordem de $P$ é $r$, claramente $c \equiv a b(\bmod r) \Leftrightarrow e([a] P,[b] P)=e(P,[c] P)$. Para alguns desses grupos, conjectura-se que o CDHP permaneça intratável. 
Seja $\mathbb{G}$ um grupo onde o problema Diffie-Hellman decisional é tratável. As observações acima levam às definições dos seguintes problemas:

- Problema Diffie-Hellman lacunar $(G D H P)$ - Resolver o CDHP em $\mathbb{G}$, possivelmente com o auxílio do oráculo que resolve o DDHP em $\mathbb{G}$.

- Problema Diffie-Hellman bilinear (BDHP) - Suponha que exista um emparelhamento $e: \mathbb{G} \times \mathbb{G} \rightarrow \mathbb{G}^{\prime}$. Dados $P,[a] P,[b] P,[c] P \in \mathbb{G}$, calcular $e(P, P)^{a b c} \in \mathbb{G}^{\prime}$.

Para cada problema acima existe um co-problema envolvendo dois grupos $\mathbb{G}_{1}$ e $\mathbb{G}_{2}$ em vez de um único grupo:

- Problema co-Diffie-Hellman decisional (co-DDHP) - Dados $P,[a] P \in \mathbb{G}_{1}$ e $Q,[b] Q \in \mathbb{G}_{2}$ onde a ordem de $P$ e $Q$ é $r$, decidir se $a \equiv b(\bmod r)$.

- Problema co-Diffie-Hellman computacional (co-CDHP) - Dados $P,[a] P \in \mathbb{G}_{1}$ e $Q \in \mathbb{G}_{2}$, calcular $[a] Q$.

- Problema co-Diffie-Hellman lacunar (co-GDHP) - Resolver o co-CDHP nos grupos $\mathbb{G}_{1}$, $\mathbb{G}_{2}$, possivelmente com o auxílio do oráculo que resolve o co-DDHP nesses grupos.

- Problema co-Diffie-Hellman bilinear (co-BDHP) - Suponha que exista um emparelhamento $e: \mathbb{G}_{1} \times \mathbb{G}_{2} \rightarrow \mathbb{G}_{3}$. Dados $P,[a] P,[b] P \in \mathbb{G}_{1}$ e $Q \in \mathbb{G}_{2}$, calcular $e(P, Q)^{a b}$.

Nota-se que o co-DDHP pode ser resolvido em grupos onde existe um emparelhamento $e: \mathbb{G}_{1} \times \mathbb{G}_{2} \rightarrow \mathbb{G}_{3}$, uma vez que, dados $P,[a] P \in \mathbb{G}_{1}$ e $Q,[b] Q \in \mathbb{G}_{2}$ onde a ordem de $P$ e $Q$ é $r$, $a \equiv b(\bmod r) \Leftrightarrow e([a] P, Q)=e(P,[b] Q)$.

A importância dos co-problemas está na possibilidade de resolver o co-GDHP num par de grupos quando não for possível resolver o GDHP num único grupo, ou definir esquemas baseados na dificuldade do co-BDHP num par de grupos quando não for possível encontrar um grupo adequado onde o BDHP seja intratável.

Dois emparelhamentos são especialmente adequados para abordar a resolução do DDHP: o emparelhamento de Weil (JOUX; NGUYEN, 2001; MENEZES; OKAMOTO; VANSTONE, 1993; MILLER, 1998; SILVERMAN, 1986) e o emparelhamento de Tate (FREY; MüLLER; RüCK, 1999; GALBRAITH; HARRISON; SOLDERA, 2002). Destes, o mais simples e flexível é o emparelhamento de Tate ${ }^{1}$.

Definição 24. Seja $E\left(\mathbb{F}_{q}\right)$ uma curva elíptica contendo um subgrupo de ordem prima r e grau de imersão $k$, e seja $\ell$ um múltiplo de $r$ que divide $q^{k}-1$. Sejam $P \in E\left(\mathbb{F}_{q}\right)[\ell], Q \in E\left(\mathbb{F}_{q^{k}}\right), f_{\ell}$

1 A definição do emparelhamento de Tate utilizada é um pouco diferente daquelas dadas em (FREY; MüLLER; RüCK, 1999), mas captura as propriedades essenciais necessárias em sistemas criptográficos baseados no problema $G D H$. 
uma função racional cujo divisor satisfaça $\left(f_{\ell}\right)=\ell(P)-\ell(O)$ e D $\sim(Q)-(O)$ um divisor com suporte disjunto do suporte de $f_{\ell}$. $O$ emparelhamento de Tate de ordem $\ell$ é a função racional $e_{\ell}: E\left(\mathbb{F}_{q}\right)[\ell] \times E\left(\mathbb{F}_{q^{k}}\right) \rightarrow \mathbb{F}_{q^{*}}^{*}$ definida por $e_{\ell}(P, Q) \equiv f_{\ell}(\mathcal{D})^{\left(q^{k}-1\right) / \ell}$.

Note-se que, como $P \in E\left(\mathbb{F}_{q}\right), f_{\ell}$ é uma função racional com coeficientes em $\mathbb{F}_{q}$. O emparelhamento de Tate satisfaz as seguintes propriedades (FREY; RüCK, 1994):

- $($ Bilinearidade $) e_{\ell}\left(P_{1}+P_{2}, Q\right)=e_{\ell}\left(P_{1}, Q\right) \cdot e_{\ell}\left(P_{2}, Q\right)$ e $e_{\ell}\left(P, Q_{1}+Q_{2}\right)=e_{\ell}\left(P, Q_{1}\right) \cdot e_{\ell}\left(P, Q_{2}\right)$ para todo $P, P_{1}, P_{2} \in E\left(\mathbb{F}_{q}\right)[\ell]$ e todo $Q, Q_{1}, Q_{2} \in E\left(\mathbb{F}_{q^{k}}\right)$. Segue daí que $e_{\ell}([a] P, Q)=$ $e_{\ell}(P,[a] Q)=e_{\ell}(P, Q)^{a}$ para todo $a \in \mathbb{Z}$.

- (Não-degeneração) $\operatorname{Se} e_{\ell}(P, Q)=1$ para todo $Q \in E\left(\mathbb{F}_{q^{k}}\right)$, então $P=O$. Alternativamente, para todo $P \neq O$ existe $Q \in E\left(\mathbb{F}_{q^{k}}\right)$ tal que $e_{\ell}(P, Q) \neq 1$.

- $\left(\right.$ Compatibilidade) $\operatorname{Se} \ell=h \ell^{\prime}, P \in E\left(\mathbb{F}_{q}\right)[\ell]$, e $Q \in E\left(\mathbb{F}_{q^{k}}\right)$, então $e_{\ell}(P, Q)=e_{\ell^{\prime}}([h] P, Q)=$ $e_{\ell^{\prime}}(P, Q)^{h}$, isto é, $f_{\ell}(\mathcal{D})^{\left(q^{k}-1\right) / \ell}=f_{\ell^{\prime}}(\mathcal{D})^{\left(q^{k}-1\right) / \ell^{\prime}}$.

A propriedade de compatibilidade permite escrever simplesmente $e(P, Q)$, subentendendose $f_{\ell}(\mathcal{D})^{\left(q^{k}-1\right) / \ell}$ para qualquer múltiplo $\ell$ de $r$ que divida $q^{k}-1$.

De modo geral, é inviável representar $e(P, Q)$ diretamente como a razão de dois polinômios. Contudo, calculando o valor do emparelhamento sob demanda mantém a complexidade computacional do emparelhamento de Tate (ou de Weil) igual à de uma multiplicação por escalar sobre a extensão da curva $E$ para o corpo finito $\mathbb{F}_{q^{k}}$. Esta é a idéia básica do algoritmo de Miller.

Sejam $P \in E\left(\mathbb{F}_{q}\right)[r]$ e $Q \in E\left(\mathbb{F}_{q^{k}}\right)$ pontos linearmente independentes, de modo que o emparelhamento de Tate satisfaz $e(P, Q) \neq 1$. O emparelhamento de Tate resolve o coDDHP definido por $P,[a] P, Q,[b] Q$. Suponha que exista um mapa de distorção eficientemente calculável $\phi:\langle P\rangle \rightarrow\langle Q\rangle$. Pode-se definir o emparelhamento derivado $\hat{e}:\langle P\rangle \times\langle P\rangle \rightarrow \mathbb{F}_{q^{k}}^{*}$ tal que $\hat{e}(P, P)=e(P, \phi(P))$. Esse emparelhamento resolve o DDHP definido por $P,[a] P,[b] P,[c] P$.

Apesar dos mapas de distorção só existem para curvas supersingulares, pode-se definir outro emparelhamento derivado $\bar{e}:\langle Q\rangle \times\langle Q\rangle \rightarrow \mathbb{F}_{q^{k}}^{*}$ tal que $\hat{e}(Q, Q)=e(\operatorname{tr}(Q),[k] Q-\operatorname{tr}(Q))$. Este outro emparelhamento derivado resolve o DDHP definido por $Q,[a] Q,[b] Q,[c] Q$.

\subsubsection{A técnica Elligator 2}

Seja $\mathbb{F}_{q}$ um corpo finito. Suponha que seja necessário mapear um valor $r \in \mathbb{F}_{q} \backslash\{0\}$, reversivel e deterministicamente, para um ponto numa curva de Montgomery $E_{M}: y^{2}=x^{3}+$ $A x^{2}+x$ sobre $\mathbb{F}_{q}, A \notin\{ \pm 2,0\}$.

A técnica Elligator (BERNSTEIN et al., 2013), ou mais precisamente Elligator 2, provê um método de obter exatamente um par de abscissas candidatas, $x_{0}$ e $x_{1}$, de tal maneira que exatamente uma delas define um ponto na curva. Especificamente, o valor $w_{b}:=x_{b}^{3}+A x_{b}^{2}+x_{b}$ 
do lado direito da equação da curva será um resíduo quadrático para uma, e apenas uma, escolha de $b \in\{0,1\}$.

A partir da observação de que $x_{b}^{3}+A x_{b}^{2}+x_{b}=x_{b}\left(x_{b}^{2}+A x_{b}+1\right)$, escolher abscissas candidatas (não nulas e distintas) $x_{0}$ e $x_{1}$ com residuosidades quadráticas opostas mas que levem ao mesmo valor (não nulo) $z:=x_{b}^{2}+A x_{b}+1$ é suficiente para garantir que $w_{0}$ and $w_{1}$ também tenham residuosidades quadráticas opostas, conforme desejado. De fato, nesse caso ocorrem $w_{0}=x_{0} z w_{1}=x_{1} z$ : as residuosidades quadráticas dos valores $w_{b}$ são as mesmas dos valores $x_{b}$ correspondentes (que são complementares entre si) se $z$ for um resíduo quadrático, e opostas (mas ainda complementares) se $z$ for um não-resíduo quadrático.

Para forçar $x_{0}$ e $x_{1}$ a terem residuosidades quadráticas opostas, define-se simplesmente $x_{1}:=u r^{2} x_{0}$ onde $u \in \mathbb{F}_{q}$ é um não-resíduo quadrático fixo, por exemplo, $u=-1$ quando $q \equiv 3(\bmod 4)$, que é o caso com os parâmetros costumeiros do SIDH. Além disso, ao impor $x_{0}^{2}+A x_{0}+1=x_{1}^{2}+A x_{1}+1$ obtém-se $\left(x_{0}+x_{1}\right)\left(x_{0}-x_{1}\right)=-A\left(x_{0}-x_{1}\right)$, e uma vez que $x_{0} \neq x_{1}$ isso se reduz a $x_{0}+x_{1}=-A$.

Combinando as duas condições $x_{0}+x_{1}=-A$ e $x_{1}=u r^{2} x_{0}$, obtêm-se $x_{0}=-A /\left(1+u r^{2}\right)$ e $x_{1}=-A-x_{0}$. Determina-se a opção correta testando o caráter quadrático de $w_{0}$ (se for um quadrado, a abscissa do ponto desejado é $x_{0}$, caso contrário a abscissa é $x_{1}$ ).

Em coordenadas projetivas de Montgomery (coordenadas $X Z$ ), isso se expressa como $t \leftarrow u \cdot r^{2}, X_{0}=-A, X_{1}=-A \cdot t, Z=1+t$ (o mesmo $Z$ para $X_{0}$ ou $X_{1}$ ). Nesse caso, a equação da curva projetiva, $Y^{2} Z=X^{3}+A X^{2} Z+X Z^{2}$, requer que $T:=4 Y^{2} Z^{2}=4\left(X^{3} Z+A X^{2} Z^{2}+X Z^{3}\right)=$ $2 X Z\left(2 X^{2}+A(2 X Z)+2 Z^{2}\right)$ seja um resíduo quadrático, o que pode ser calculado como $X_{2} \leftarrow X^{2}$, $Z_{2} \leftarrow Z^{2}, W \leftarrow(X+Z)^{2}-X_{2}-Z_{2}=2 X Z, T \leftarrow W \cdot\left(2\left(X_{2}+Z_{2}\right)+A \cdot W\right)$.

\subsubsection{ECDH - Elliptic Curve Diffie-Hellman}

O protocolo Diffie-Hellman de negociação de chaves é o mais tradicional em toda a criptografia de chaves públicas (DIFFIE; HELLMAN, 1976). A versão do protocolo usando curvas elípticas é descrito a seguir.

A troca de chaves será realizada por duas entidades: Alice e Bob. Segue-se a convenção de usar os subscritos $A$ e $B$ para Alice e Bob, respectivamente.

Ambos escolhem valores privados $s_{A} \stackrel{\$}{\leftarrow}(\mathbb{Z} / r \mathbb{Z})^{*}$ e $s_{B} \stackrel{\$}{\leftarrow}(\mathbb{Z} / r \mathbb{Z})^{*}$ respectivamente, e calculam os valores públicos correspondentes $Y_{A}=\left[s_{A}\right] G$ e $Y_{B}=\left[s_{B}\right] G$. Em seguida transmitem um ao outro seus valores públicos, que são pontos da curva. $\mathrm{O}$ valor comum (também um ponto da curva) é calculado por $A$ como $K=\left[s_{A}\right] Y_{B}$, e por $B$ como $K=\left[s_{B}\right] Y_{A}$, obtendo nos dois casos $K=\left[s_{A} s_{B}\right] G$. Esse valor comum pode ser então usado como chave simétrica num esquema de cifração convencional. 


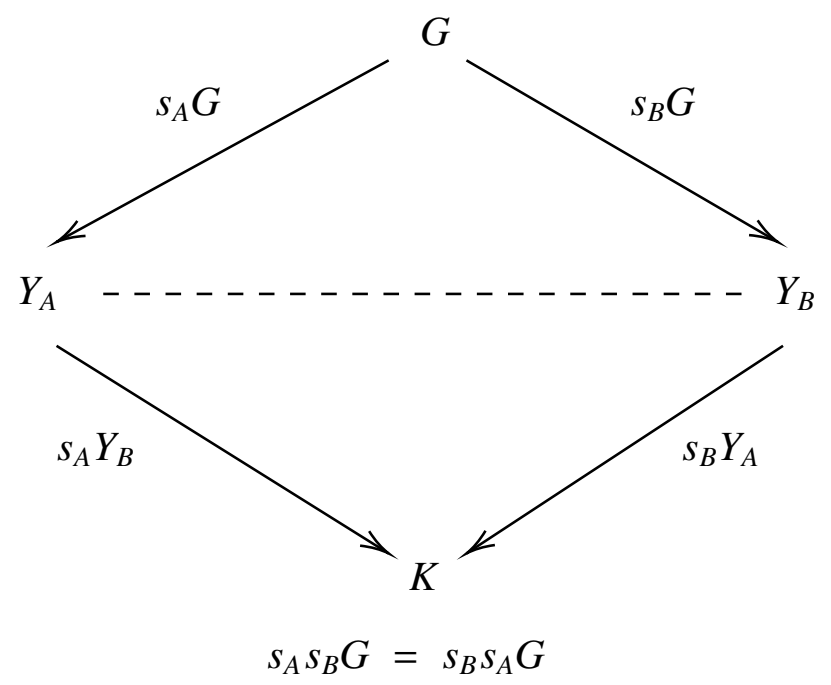

Figura 12 - Esquematização do protocolo de troca de chaves $E C D H$.

Considere a curva da Figura 11 com o gerador $G=(1,1)$. A ordem da curva é $r=11$. Suponha que Alice escolha o valor privado $s_{A}=4$, obtendo o ponto público $Y_{A}=[4] G=(4,5)$; da mesma forma, suponha que Bob escolha $s_{B}=9$, obtendo $Y_{B}=[9] G=(2,3)$. Após a troca de pontos públicos, Alice calcula o ponto comum $K=\left[s_{A}\right] Y_{B}=[4](2,3)=(6,5)$, enquanto Bob calcula o mesmo ponto $K=\left[s_{B}\right] Y_{A}=[9](4,5)=(6,5)$. É fácil ver que o valor de $K$ está correto: $K=[4]([9] G)=[9]([4] G)=[36 \bmod 11] G=[3] G=(6,5)$.

\subsection{Isogenias de curvas elípticas}

A noção de isogenia, bem estabelecida em geometria algébrica, dá origem a certos problemas computacionais que se mostraram úteis para aplicações criptográficas. Expõem-se a seguir os conceitos fundamentais envolvidos e os algoritmos essenciais para a criação e cálculo de isogenias entre curvas elípticas.

Definição 25 (Isogenia de curvas elípticas). Seja $\mathbb{F}$ um corpo com característica char $(\mathbb{F})>3$ e sejam E e $E^{\prime}$ curvas elípticas sobre $\mathbb{F}$. Uma isogenia entre E e E' é um homomorfismo $\phi: E(\overline{\mathbb{F}}) \rightarrow E^{\prime}(\overline{\mathbb{F}})$ definido pelas funções racionais $r, s \in \mathbb{F}(x)$ como $\phi(x, y)=(r(x), y \cdot s(x))$ satisfazendo $\phi\left(O_{E}\right)=\phi\left(O_{E^{\prime}}\right)$, onde $O_{E}$ e $O_{E^{\prime}}$ são os elementos neutros ("pontos no infinito") das curvas $E_{A}$ e $E_{B}$, respectivamente. Se tal mapa existe, então as curvas são ditas isógenas.

Proposição 1. Duas curvas elípticas são isomórfas sobre $\overline{\mathbb{F}}$ se e somente se possuírem o mesmo j-invariante. (SILVERMAN, 1986, Capítulo III, Seção 1, Proposição 1.4(b))

Definição 26 (Grau de uma isogenia). Considere a função racional $r(x)$ que define uma isogenia entre curvas escrita na forma $r(x)=n(x) / d(x)$ para $n, d \in \mathbb{F}[x] \operatorname{com} \operatorname{gcd}(n, d)=1$. O grau de uma isogenia $\phi$ é dado por max $\{\operatorname{deg}(n), \operatorname{deg}(d)\}$. Uma isogenia $\phi$ de grau deg $(\phi)=g$ é chamada de g-isogenia. 
Definição 27 (Isogenia separável). Considere a função racional $r(x)$ que define uma isogenia entre curvas. Se a derivada $r^{\prime}(x) \not \equiv 0$ (i.e. $r(x)$ é não-constante), $\phi$ é chamada de isogenia separável. Nesse caso o grau da isogenia equivale o número de elementos no núcleo: $\operatorname{deg}(\phi)=$ $|\operatorname{ker}(\phi)|=\left|\left\{P \in E \mid \phi(P)=O_{E}\right\}\right|$.

Mesmo quando se sabe que duas curvas são isógenas, construir uma isogenia entre elas é, via de regra, um problema NP-difícil. Se as curvas forem ordinárias, esse problema pode ser resolvido por um computador quântico em tempo subexponencial (CHILDS; JAO; SOUKHAREV, 2014), em virtude de o grupo de classe do anel de endomorfismos dessas curvas ser abeliano. Para curvas supersingulares, o grupo de classe (Definição 13) não é comutativo (mais precisamente, é uma ordem numa álgebra de quatérnions (Definição. 14), e não se conhece nenhum algoritmo quântico que tenha complexidade subexponencial para esse problema (JAO; DeFEO, 2011; DeFEO; JAO; PLÛT, 2014). Existe um algoritmo exponencial denominado claw-finding que possui complexidade $O\left(p^{\frac{1}{4}}\right)$ no cenário clássico e $O\left(p^{\frac{1}{6}}\right)$ no quântico (JAQUES; SCHANCK, 2019).

O Teorema 28 (SILVERMAN, 1986, Capítulo V, Seção 4, Teorema 4.1 (c)) mostra que o número de elementos do um subconjunto de curvas supersingulares em um corpo $F_{q}$ é linear.

Teorema 28. Seja $\mathbb{F}_{q}$ um corpo finito com característica $p \geq 3$. Então o polinômio $H_{p}(t)$ possui raízes distintas em $\overline{\mathbb{F}}_{q}$. Existe uma curva supersingular de característica 3 , e para $p \geq 5$, o número de curvas elípticas supersingulares (equivalentes por isomorfismo em $\overline{\mathbb{F}}_{q}$ ) é:

$$
\left[\frac{p}{12}\right]+\left\{\begin{array}{llll}
0 & \text { se } & p \equiv 1 & (\bmod 12) \\
1 & \text { se } & p \equiv 5 & (\bmod 12) \\
1 & \text { se } & p \equiv 7 & (\bmod 12) \\
2 & \text { se } & p \equiv 11 & (\bmod 12)
\end{array}\right.
$$

Assim, se $p$ cresce exponencialmente, o crescimento do subgrupo em questão também será exponencial.

\subsubsection{Cálculo de isogenias de grau suave}

Conforme indicado acima, calcular isogenias entre curvas elípticas supersingulares sobre corpos finitos é um problema computacional conjecturado como difícil, com custo exponencial no grau de isogenia.

No entanto, em 1971 Vélu descobriu fórmulas de tempo polinomial para calcular isogenias de baixo grau a partir de um ponto no núcleo desejado da isogenia utilizando-se curvas no modelo de Weierstrass. Para $K:=\mathbb{F}_{q}, E / K: y^{2}=x^{3}+a x+b$ e núcleo $\phi=\left\{\infty,\left(x_{2}, 0\right)\right\}$, por exemplo, as fórmulas de Vélu para 2-isogenias produzem a curva $E^{\prime} / K: y^{2}=x^{3}+a^{\prime} x+b^{\prime}$ com 
$a^{\prime}:=a-5\left(3 x_{2}^{2}+a\right), b^{\prime}:=b-7 x_{2}^{2}\left(3 x_{2}^{2}+a\right)$, e a 2-isogenia

$$
\phi_{2}(x, y):=\left(x+\frac{3 x_{2}^{2}+a}{x-x_{2}}, y\left(1-\frac{3 x_{2}^{2}+a}{\left(x-x_{2}\right)^{2}}\right)\right)=\left(f(x), y \cdot f^{\prime}(x)\right)
$$

onde $f(x):=x+\frac{3 x_{2}^{2}+a}{x-x_{2}}$. Conforme o grau da isogenia aumenta, essas fórmulas tornam-se cada vez mais complexas, com o custo é exponencial em virtude de a fórmula envolver um termo adicional para cada par de pontos $(P,-P)$ no núcleo da isogenia. Como o núcleo é um subgrupo, ou seja, cada elemento do núcleo tem a forma $[\alpha] G$ onde $G$ é um gerador e $\alpha$ é um inteiro de $k$ bits, sua cardinalidade é da ordem de $2^{k}$. Para a mesma curva do exemplo anterior e núcleo $\phi=\left\{\infty,\left(x_{3}, \pm y_{3}\right)\right\}$, as fórmulas para 3-isogenias produzem a curva $E^{\prime} / K: y^{2}=x^{3}+a^{\prime} x+b^{\prime}$ com $a^{\prime}:=a-10\left(3 x_{3}^{2}+a\right), b^{\prime}:=b-14\left(2 y_{3}^{2}+x_{3}\left(3 x_{3}^{2}+a\right)\right)$, e a isogenia

$$
\phi_{3}(x, y):=\left(x+\frac{6 x_{3}^{2}+2 a}{x-x_{3}}+\frac{4 y_{3}^{2}}{\left(x-x_{3}\right)^{2}}, y\left(1-\frac{6 x_{3}^{2}+2 a}{\left(x-x_{3}\right)^{2}}-\frac{8 y_{3}^{2}}{\left(x-x_{3}\right)^{3}}\right)\right)=\left(f(x), y \cdot f^{\prime}(x)\right)
$$

, onde $f(x):=x+\frac{6 x_{3}^{2}+2 a}{x-x_{3}}+\frac{4 y_{3}^{2}}{\left(x-x_{3}\right)^{2}}$.

Em 2017, Costello e Hisil (COSTELLO; HISIL, 2017) encontraram fórmulas mais simples, eficientes e que só consideram a coordenada $x$ para o cálculo de isogenias em curvas no modelo de Montgomery.

Teorema 29 (Costello-Hisil, 2017). Seja $E / K: B y^{2}=x^{3}+A x^{2}+x$ uma curva no modelo de Montgomery e seja $P \in E(\bar{K})$ uma curva de ordem $\ell=2 t+1$ para algum $t$. Sejam também $\sigma:=\sum_{i=1}^{t} x_{[i] P}, \tilde{\sigma}:=\sum_{i=1}^{t} 1 / x_{[i] P}, \pi:=\prod_{i=1}^{t} x_{[i] P}$.

Então a curva $E^{\prime} / K: B^{\prime} y^{2}=x^{3}+A^{\prime} x^{2}+x \operatorname{com} A^{\prime}:=(A-6 \sigma+6 \tilde{\sigma}) \cdot \pi^{2} e B^{\prime}:=B \cdot \pi^{2}$ éo codomínio da $\ell$-isogenia $\phi: E \rightarrow E^{\prime}$ definida por $\phi(x, y)=\left(f(x), y \cdot f^{\prime}(x)\right)$ com núcleo $\phi=\langle P\rangle$ e $f(x):=x \cdot \prod_{i=1}^{t}\left(\frac{x \cdot x_{[i] p^{-1}}}{x-x_{[i] P}}\right)^{2}$

Para $E / K: y^{2}=x^{3}+A x^{2}=x$ e núcleo $\phi_{2}=\left\{\infty,\left(x_{2}, 0\right)\right\}$, por exemplo, as fórmulas de Costello-Hisil para 2-isogenias produzem a curva $E^{\prime} / K: y^{2}=x^{3}+A^{\prime} x+x \operatorname{com} A^{\prime}:=4 x_{2}-2$, e a 2-isogenia

$$
\phi_{2}(x, y)=\left(-x \cdot \frac{x_{2} x-1}{x-x_{2}}, y\left(-x_{2} \frac{\left(x^{2}-2 x_{2} x+1\right)}{\left(x-x_{2}\right)^{2}}\right)\right)=\left(f(x), y \cdot f^{\prime}(x)\right)
$$

onde $f(x):=-x \cdot \frac{x_{2} x-1}{x-x_{2}}$. No caso em que $\ell=3$, essas formulas produzem para o núcleo $\phi_{3}=\left\{\infty,\left(x_{3}, \pm y_{3}\right)\right\}$ a curva $E^{\prime} / K: y^{2}=x^{3}+A^{\prime} x+x \operatorname{com} A^{\prime}:=A x_{3}-6 x_{3}^{2}+2$, e a 3-isogenia

$$
\phi_{3}(x, y)=\left(x \cdot\left(\frac{x_{3} x-1}{x-x_{3}}\right)^{2}, y\left(\frac{3 x^{2} x_{3}^{3}-x\left(x^{2}+4\right) x_{3}^{2}+x_{3}+x}{\left(x-x_{3}\right)^{3}}\right)\right)=\left(f(x), y \cdot f^{\prime}(x)\right)
$$

onde $f(x)=x \cdot\left(\frac{x_{3} x-1}{x-x_{3}}\right)^{2}$. Note que tanto para 2 quanto para 3-isogenias, a função $f(x)$ independe de $y$. 
Essas fórmulas podem ser compostas/iteradas polinomialmente para produzir isogenias de grau suave muito maior, especificamente de grau $\ell^{m}$, onde $\ell$ é um primo pequeno.

Naturalmente, isso pressupõe que o núcleo contém um ponto de ordem $\ell^{m}$, mas é possível escolher parâmetros (especificamente, tomando um corpo finito subjacente $\mathbb{F}_{q}$ com $\ell^{m} \mid q+1$ ) para garantir que este seja realmente o caso.

$\mathrm{O}$ método de construção funciona da seguinte maneira para $0 \leqslant j<m$ :

- Dado um ponto $P_{j} \in E_{j}$ de ordem $\ell^{m-j}$ no núcleo desejado da isogenia, obtenha o ponto $P_{j}^{\prime}:=\left[\ell^{m-j-1}\right] P_{j}$ de ordem $\ell$.

- Aplique a fórmula de Vélu ao ponto $P_{j}^{\prime}$ para calcular uma isogenia $\phi_{j+1}: E_{j} \rightarrow E_{j+1}$ e (os coeficientes d)a curva isógena $E_{j+1}$ tal que $\phi_{j+1}\left(P_{j}^{\prime}\right)=O_{E_{j+1}}$.

- Calcule a imagem $P_{j+1}:=\phi_{j+1}\left(P_{j}\right) \in E_{j+1}$ de $P_{j}$ pela isogenia $\phi_{j+1}$. Note que $P_{j+1}$ é um ponto de ordem $\ell^{m-j-1}$ :

○ $P_{j+1}$ é um ponto de $\ell^{m-j-1}$-torção, uma vez que $\left[\ell^{m-j-1}\right] P_{j+1}=\left[\ell^{m-j-1}\right] \phi_{j+1}\left(P_{j}\right)=$ $\phi_{j+1}\left(\left[\ell^{m-j-1}\right] P_{j}\right)=\phi_{j+1}\left(P_{j}^{\prime}\right)=O_{E_{j+1}}$, onde a última igualdade vale porque $P_{j}^{\prime}$ é, por definição, um ponto do núcleo.

- Além disso, em virtude de estar na $\ell^{m-j-1}$-torção, a ordem de $P_{j+1}$ só pode ser uma potência de $\ell$, mas se fosse uma potência menor $k<m-j-1$, ou seja, $\left[\ell^{k}\right] P_{j+1}=O_{E_{j+1}}$, então $\left[\ell^{k}\right] P_{j+1}=\left[\ell^{k}\right] \phi_{j+1}\left(P_{j}\right)=\phi_{j+1}\left(\left[\ell^{k}\right] P_{j}\right)=O_{E_{j+1}}$, significando que $\left[\ell^{k}\right] P_{j} \neq O_{E_{j}}$ está no núcleo de $\phi_{j+1}$. Mas isso é impossível sob a hipótese indutiva de que $P_{j}$ é um ponto de ordem $\ell^{m-j}>\ell$.

- Repita o processo a partir da nova curva $E_{j+1}$ e do ponto $P_{j+1}$ de ordem $\ell^{m-1}$.

O método acima tem complexidade quadrática no grau $m$ da isogenia, ou seja, $O\left(\mathrm{~m}^{2}\right)$ operações aritméticas no corpo finito, uma vez que precisa ser repetido para $P_{1}, \ldots, P_{m}$, e cada passo incorre uma multiplicação de ponto por um escalar de tamanho $O(m)$ bits. Jao e De Feo projetaram um algoritmo eficiente para o processo acima, de complexidade $O(m \log m)$ baseado em programação dinâmica.

\subsubsection{Fórmulas de Vélu}

Conforme indicado acima, as fórmulas gerais de Vélu são extremamente complexas (WASHINGTON, 2008, seção 12.3, teorema 12.16) e não têm utilidade criptográfica imediata nesse forma.

As fórmulas Costello-Hisil são muito mais efetivas, e admitem uma implementação particularmente eficiente nas coordenadas projetivas típicas do modelo de Montgomery para $\ell=$ $2, \ell=3$, e também para $\ell=4$ (mais eficiente que duas aplicações sucessivas das fórmulas para 
$\ell=2$ ), quando a curva elíptica admite um modelo de Montgomery $E_{a}: Y^{2} Z=X^{3}+a X^{2} Z+X Z^{2}$. A adoção de uma curva desse tipo é especialmente útil para aplicações criptográficas, devido às fórmulas de Montgomery em coordenadas $[X: Z]$ para a lei de grupo, mais eficientes que as fórmulas de qualquer outro modelo conhecido.

Curiosamente, a fórmula "oficial" da especificação do criptossistema SIKE não é a mais simples possível para $\ell=4$. Apresenta-se abaixo uma fórmula nova e ligeiramente mais simples, que economiza uma adição no corpo finito. Obviamente, essa economia não vai melhorar substancialmente o cálculo de isogenias de grau suave maior, mas parece ser a mais simples conhecida.

Por conveniência, seja $a_{24}:=(a+2) / 4$ (esta forma do coeficiente da curva ocorre diretamente na lei de grupo). As fórmulas de Vélu produzem diretamente o coeficiente $a_{24}^{\prime}:=$ $\left(a^{\prime}+2\right) / 4$ da curva isógena $E_{a^{\prime}}$ em forma projetiva $\left[A_{24}^{\prime}: C_{24}^{\prime}\right] \sim\left[a_{24}^{\prime}: 1\right]$, com o numerador e o denominador separados para evitar o elevado custo computacional de uma inversão no corpo finito.

Essas fórmulas são as seguintes:

- Para $\ell=2$, seja $\left[X_{2}: Z_{2}\right] \in E_{a}$ um ponto de ordem 2 no núcleo desejado. Então

$$
\begin{gathered}
{\left[A_{24}^{\prime}: C_{24}^{\prime}\right]=\left[X_{2}^{2}-Z_{2}^{2}: Z_{2}^{2}\right],} \\
\phi_{2}([X: Z])=\begin{array}{r}
{\left[X\left(\left(X_{2}+Z_{2}\right)(X-Z)+\left(X_{2}-Z_{2}\right)(X+Z)\right):\right.} \\
\left.Z\left(\left(X_{2}+Z_{2}\right)(X-Z)-\left(X_{2}-Z_{2}\right)(X+Z)\right)\right] .
\end{array}
\end{gathered}
$$

- Para $\ell=3$, seja $\left[X_{3}: Z_{3}\right] \in E_{a}$ um ponto de ordem 3 no núcleo desejado. Então

$$
\begin{gathered}
\left(\left[A_{24}^{\prime}: C_{24}^{\prime}\right]=\left[\left(X_{3}+Z_{3}\right)\left(Z_{3}-3 X_{3}\right)^{3}: 16 X_{3} Z_{3}^{3}\right],\right. \\
\phi_{3}([X: Z])=\left[X\left(X_{3} X-Z_{3} Z\right)^{2}: Z\left(Z_{3} X-X_{3} Z\right)^{2}\right] .
\end{gathered}
$$

- Para $\ell=4$, seja $\left[X_{4}: Z_{4}\right] \in E_{a}$ um ponto de ordem 4 no núcleo desejado. Então

$$
\begin{gathered}
{\left[\mathbf{A}_{\mathbf{2 4}}^{\prime}: \mathbf{C}_{\mathbf{2 4}}^{\prime}\right]=\left[\mathbf{X}_{\mathbf{4}}^{\mathbf{4}}: \mathbf{Z}_{\mathbf{4}}^{4}\right],} \\
\phi_{4}([X: Z])=\left[\begin{array}{l}
X\left(2 X_{4} Z_{4} Z-X\left(X_{4}^{2}+Z_{4}^{2}\right)\right)\left(X_{4} X-Z_{4} Z\right)^{2}: \\
\left.Z\left(2 X_{4} Z_{4} X-Z\left(X_{4}^{2}+Z_{4}^{2}\right)\right)\left(Z_{4} X-X_{4} Z\right)^{2}\right] .
\end{array}\right.
\end{gathered}
$$

Esta é a fórmula ligeiramente melhorada prometida acima. A fórmula "oficial" na especificação do sistema SIKE calcula $\left[A_{24}^{\prime}: C_{24}^{\prime}\right]=\left[4 X_{4}^{4}: 4 Z_{4}^{4}\right]$.

\subsubsection{SIDH - Supersingular Isogeny Diffie-Helman}

A troca de chaves será realizada por duas entidades: Alice e Bob. Segue-se a convenção de usar os subscritos $A$ e $B$ para Alice e Bob, respectivamente. Por exemplo, a isogenia secreta 
$\phi_{A}$ é computada por Alice e seus parâmetros públicos são denotados pelos pontos $P_{A}, Q_{A}$ e a curva $E_{A}$. Da mesma forma, a isogenia de Bob é denotada por $\phi_{B}$ e seus parâmetros públicos são $P_{B}, Q_{B}, E_{B}$.

O protocolo de acordo de chaves tem como parâmetros inicias um primo $p=\ell_{A}^{e_{A}} \ell_{B}^{e_{B}} \cdot f-1$, uma curva inicial $E_{0}$ e bases fixas $\left\{P_{A}, Q_{A}\right\},\left\{P_{B}, Q_{B}\right\}$ para Alice e Bob.

Alice inicialmente:

- Gera escalares $m_{A}, n_{A} \in \mathbb{Z} / \ell_{A}^{e_{A}} \mathbb{Z}$ e calcula o ponto $R_{A}:=m_{A} P_{A}+n_{A} Q_{A}$;

- Calcula o mapa de isogenia $\phi_{A}: E_{0} \rightarrow E_{A}:=E_{0} /\left\langle R_{A}\right\rangle$ através das fórmulas de Vélu;

- Aplica o mapa obtido nos pontos públicos de Bob, obtendo $\phi_{A}\left(P_{B}\right)$ e $\phi_{A}\left(Q_{B}\right)$;

- Envia a curva isógena $E_{A}$ e os pontos $\phi_{A}\left(P_{B}\right)$ e $\phi_{A}\left(Q_{B}\right)$ para Bob;

Bob realiza processo análogo à Alice, porém em sua torção.

Após receber a curva $E_{B}$ e os pontos $\phi_{B}\left(P_{A}\right)$ e $\phi_{B}\left(Q_{A}\right)$, Alice:

- Calcula o ponto $S_{A}:=m_{A}\left(\phi_{B}\left(P_{A}\right)\right)+n_{A}\left(\phi_{B}\left(P_{A}\right)\right)$;

- Calcula o mapa de isogenia $\phi_{A}^{\prime}: E_{B} \rightarrow E_{A B}:=E_{B} /\left\langle S_{A}\right\rangle$ através das fórmulas de Vélu;

- Calcula o $j$-invariante $K:=j\left(E_{A B}\right)$

Novamente, Bob realiza processo análogo à Alice e obtém como valor final seu $j$ invariante $K:=j\left(E_{B A}\right)$.

Por construção, $E_{A B}=\phi_{B}^{\prime}\left(\phi_{A}\left(E_{0}\right)\right)=\phi_{A}^{\prime}\left(\phi_{B}\left(E_{0}\right)\right)=E_{0} /\left\langle m_{A} P_{A}+n_{A} Q_{A}, m_{B} P_{B}+n_{B} Q_{B}\right\rangle$, e $K=j\left(E_{A B}\right)=j\left(E_{B A}\right)$ forma o segredo compartilhado.

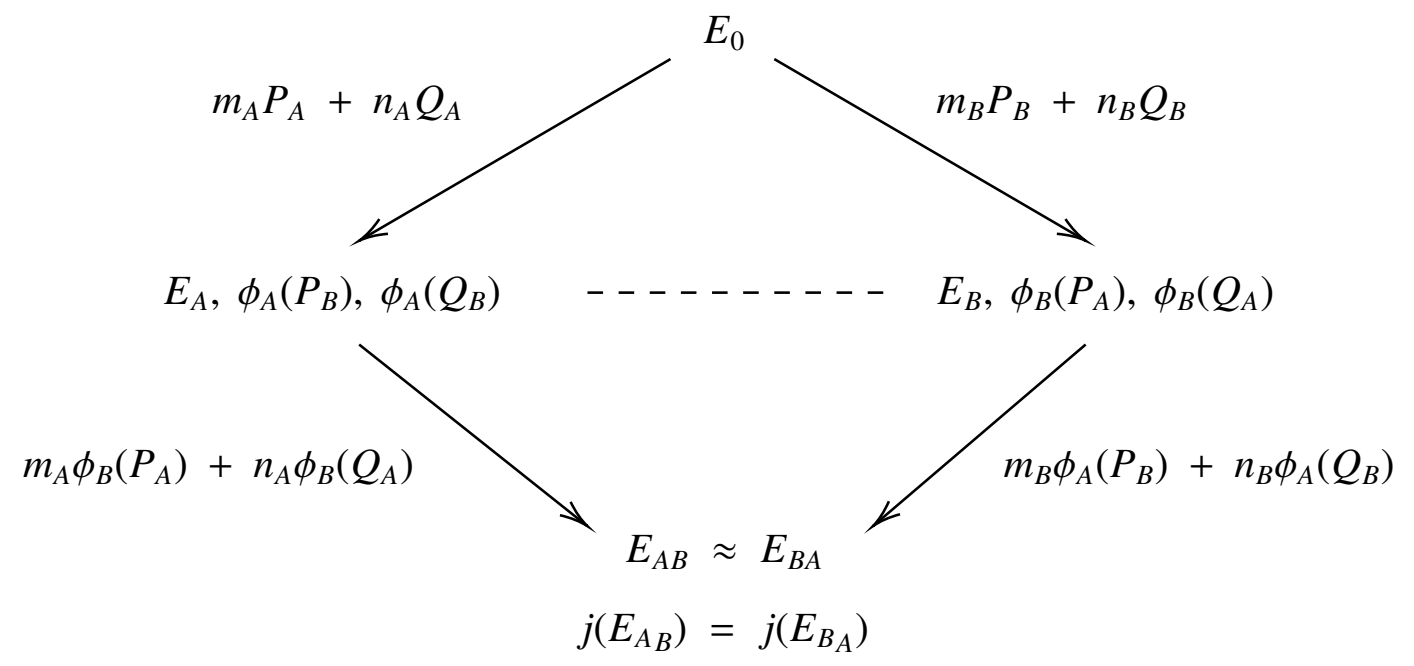

Figura 13 - Esquematização do protocolo de troca de chaves SIDH. 
O SIKE, por sua vez, é apenas uma instanciação eficiente de SIDH, onde a curva inicial é $E_{0} / \mathbb{F}_{p^{2}}: y^{2}=x^{3}+6 x^{2}+x$ e todas as curvas envolvidas estão no modelo de Montgomery com coeficiente $B=1$.

\subsubsection{Exemplo numérico}

Considere o exemplo simplificado onde $p=2^{4} \cdot 3^{3}-1=431$, uma a curva inicial $E_{0} / \mathbb{F}_{p^{2}}: y^{2}=x^{3}+6 x+x$ e as bases fixas $\left\{P_{A}, Q_{A}\right\}=\{(176+190 i, *),(107+306 i, *)\} \mathrm{e}$ $\left\{P_{B}, Q_{B}\right\}=\{(81, *),(286, *)\}$ de Alice e Bob, respectivamente.

Pelo Teorema 28, sabe-se que o número de elementos do subconjunto de $F_{p}^{2}$ é dado por $\lfloor 431 / 12\rfloor+2=37$. Cada elemento corresponde uma classe de equivalência com um ou mais cuvas isógenas e portanto com o mesmo $j$-invariante. $\mathrm{O}$ subconjunto com seu $j$-invariante correspondente é mostrado na figura:

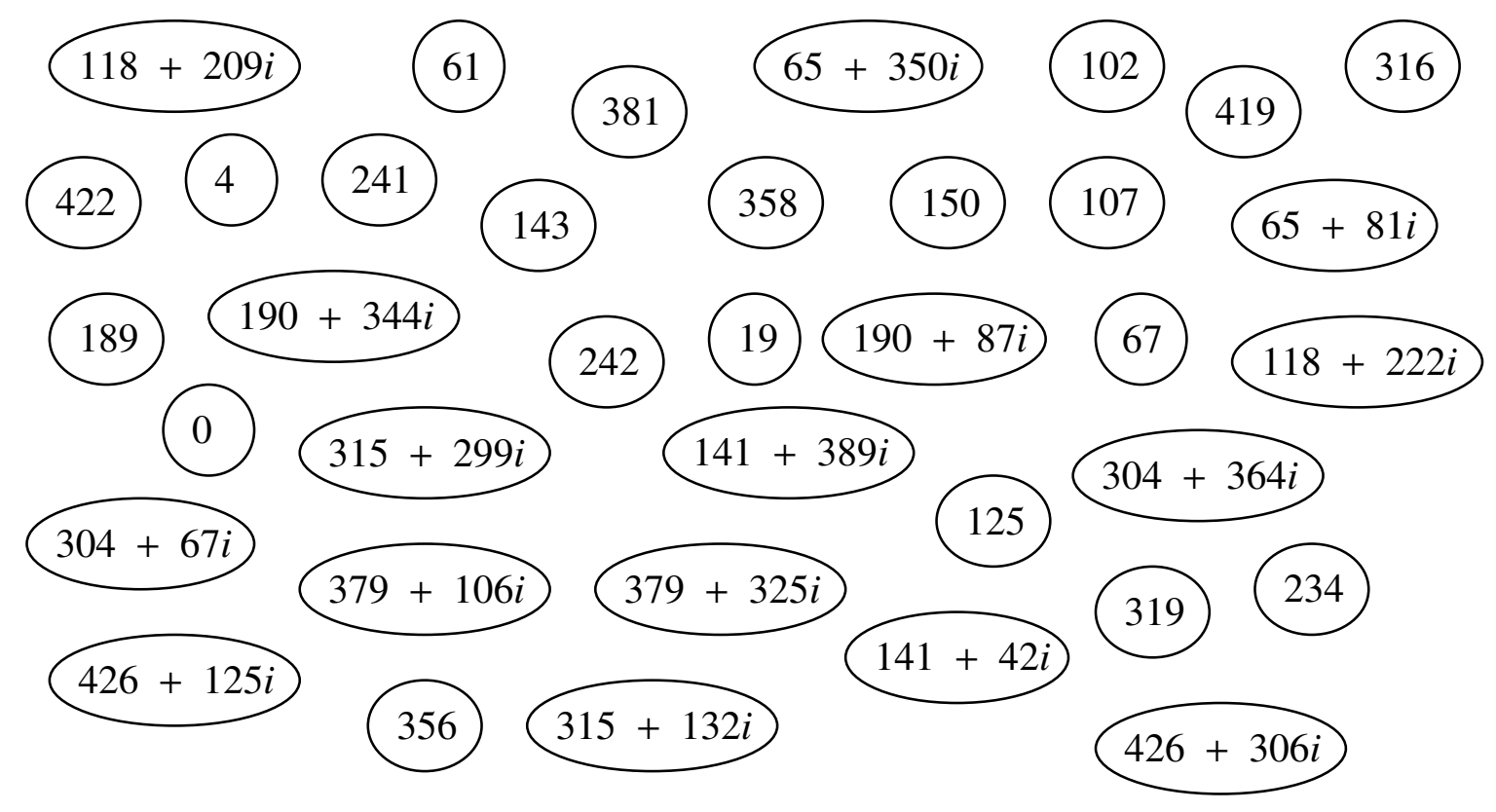

Figura 14 - $\mathrm{O}$ conjunto dos $37 \mathrm{j}$-invariantes de curvas supersingulares em $\mathbb{F}_{431^{2}}$.

Fonte: Costello (2019)

O valor do $j$-invariante da curva inicial $E_{0} / \mathbb{F}_{p^{2}}$ é dado por:

$$
j\left(E_{0}\right)=256 \frac{\left(A^{2}-3\right)^{3}}{\left(A^{2}-4\right)}=256 \frac{\left(6^{2}-3\right)^{3}}{\left(6^{2}-4\right)} \quad(\bmod 431)=19
$$

Alice escolhe aleatóriamente um escalar $s_{A}=1 \in \mathbb{F}_{p}^{2}$ e obtém o ponto $P_{A}+\left[s_{A}\right] Q_{A}=$ $(176+190 i, *)+[1](107+306 i, *)=(243+251 i, *)$. Utilizando-se das fórmulas para cálculo de isogenias, ela obtém o mapa $\phi_{A}$ e a curva $E_{A}: y^{2}=x^{3}+(10+183 i) x^{2}+x \operatorname{com} j\left(E_{A}\right)=190+87 i$ a partir do núcleo $\operatorname{ker} \phi_{A}=\langle(243+251 i, *)\rangle$ e da curva inicial. Por fim, aplica sua transformação 
nos pontos $\left\{P_{B}, Q_{B}\right\}$, obtém $\left\{\phi_{A}\left(P_{B}\right), \phi_{B}\left(Q_{B}\right)\right\}=\{(339+276 i, *),(297-171 * i, *)\} \in E_{A}$ e envia para Bob.

Analogamente, Bob escolhe aleatóriamente um escalar $s_{B}=23 \in \mathbb{F}_{p}^{2}$ e obtém o ponto $P_{B}+\left[s_{B}\right] Q_{B}=(81, *)+[23](286, *)=(398+279 i, *)$, o mapa $\phi_{B}$ e a curva $E_{B}: y^{2}=$ $x^{3}+(226+412 i) x^{2}+x \operatorname{com} j\left(E_{B}\right)=379+106 i$. Por fim, aplica sua transformação nos pontos $\left\{P_{A}, Q_{A}\right\}$, obtém $\left\{\phi_{B}\left(P_{A}\right), \phi_{B}\left(Q_{A}\right)\right\}=\{(339+234 i, *),(297-21 i, *)\} \in E_{B}$ e envia para Alice.

$\mathrm{Na}$ etapa final, Alice e Bob aplicam as fórmulas de cálculo de isogenia nos núcleos $\operatorname{ker} \phi_{A B}=\left\langle\phi_{B}\left(P_{A}\right)+\left[s_{A}\right] \phi_{B}\left(Q_{A}\right)\right\rangle$ e $\operatorname{ker} \phi_{B A}=\left\langle\phi_{A}\left(P_{B}\right)+\left[s_{B}\right] \phi_{A}\left(Q_{B}\right)\right\rangle$ e suas respectivas curvas $E_{A}$ e $E_{B}$. Como resultado, obtém-se $E_{A B}: y^{2}=x^{3}+A^{\prime} x^{2}+x, E_{B A}: y^{2}=x^{3}+B^{\prime} x^{2}+x$ com $A^{\prime}=B^{\prime}=2+251 i$ e mesmo j-invariante $j\left(E_{A B}\right)=j\left(E_{B A}\right)=426+306 i$.

O processo descrito pode ser visualizado como um trajeto na Figura 14 relacionando os j-invariantes obtidos em cada curva.

\subsubsection{O problema da compressão de chaves}

Apesar de apresentarem o menor tamanho de chaves dentre as alternativas pós-quânticas, esquemas baseados em isogenias ainda possuem chaves expressivamente maiores em relação ao que é adotado atualmente por criptossistemas clássicos. Uma chave pública do protocolo ECDH, por exemplo, é dado por um ponto $P \in E\left[\mathbb{F}_{p}\right]$ que pode ser representado por sua coordenada $x_{P} \in \mathbb{F}_{p}$, e portanto seu tamanho ocupa $\approx \lg p$ bits.

Os artigos originais do SIDH (JAO; DeFEO, 2011; DeFEO; JAO; PLÛT, 2014) representam as chaves públicas pela tripla:

$$
\left(E^{\prime}, \phi(P), \phi(Q)\right)
$$

onde $E^{\prime} / \mathbb{F}_{p^{2}}: y^{2}=x^{3}+a x+b$ é uma curva supersingular no modelo reduzido de Weierstrass e $\phi(P), \phi(Q) \in E^{\prime}$ correspondem às imagens das bases públicas fixas sob a isogenia secreta $\phi$.

A curva $E^{\prime}$ pode ser representada por seus coeficientes $a, b \in \mathbb{F}_{p^{2}}$, necessitando de $2 \lg p^{2}=4 \lg p$ bits. Os pontos $\phi(P), \phi(Q) \in E^{\prime}$ podem ser representados somente por sua coordenada $x$ (mais um bit adicional para indicar o sinal da coordenada $y$ ). Como $x_{\phi(P)}, x_{\phi(Q)} \in \mathbb{F}_{p^{2}}$, sua representação também ocupa $2 \lg p^{2}=4 \lg p$ bits, totalizando $8 \lg \mathbf{p}$ bits por chave pública.

Entretanto, pode-se reduzir o tamanho da chave pública utilizando-se uma curva inicial no modelo de Montgomery $E^{\prime} / \mathbb{F}_{p^{2}}: y^{2}=x^{3}+a^{\prime} x^{2}+x$, conforme utilizado pelo SIKE. Com apenas um escalar $a^{\prime} \in \mathbb{F}_{p^{2}}$ necessário para representar a curva inicial, a chave pública passa a ocupar $\mathbf{6} \lg \mathbf{p}$ bits.

Azarderakhsh et al. (AZARDERAKHSH et al., 2016) observaram que, como $\phi(P) \mathrm{e}$ $\phi(Q)$ são pontos de $2^{m}$ ou $3^{n}$-torção com $2^{m} \approx 3^{m}$ e $p=2^{m} 3^{n}-1$, então $m \approx \frac{1}{2} \lg p$. Além disso, qualquer ponto de uma $\ell^{e}$-torção pode ser expresso em relação a uma base pública $\left(R_{1}, R_{2}\right)$ na forma $\phi(P)=\left[a_{0}\right] R_{1}+\left[b_{0}\right] R_{2}$, onde $a_{0}$ e $b_{0}$ são inteiros de $m$ bits. O mesmo pode ser 
feito com $\phi(Q)=\left[a_{1}\right] R_{1}+\left[b_{1}\right] R_{2}$. A chave pública passa a ser representada pela quíntupla $\left(a^{\prime}, a_{0}, b_{0}, a_{1}, b_{1}\right) \in \mathbb{F}_{p^{2}} \times\left(\mathbb{Z} / 2^{m} \mathbb{Z}\right)^{4}$, que ocupa $2 \lg p+4 \cdot \frac{1}{2} \lg p=\mathbf{4} \lg \mathbf{p}$ bits.

Os pontos $\phi(P)$ e $\phi(Q)$ são somente necessários para calcular o núcleo

$$
\langle\phi(P)+[s] \phi(Q)\rangle=\left\langle\left[a_{0}\right] R_{1}+\left[b_{0}\right] R_{2}+[s]\left(\left[a_{1}\right] R_{1}+\left[b_{1}\right] R_{2}\right)\right\rangle=\left\langle\left[a_{0}+s a_{1}\right] R_{1}+\left[b_{0}+s b_{1}\right] R_{2}\right\rangle
$$

Assumindo sem perda de generalidade que o núcleo acima pode ser calculado como

$$
\left\langle\left[a_{0}\right]\left[1+s \frac{a_{1}}{a_{0}}\right] R_{1}+\left[\frac{b_{0}}{a_{0}}+s \frac{b_{1}}{a_{0}}\right] R_{2}\right\rangle=\left\langle\left[1+s \frac{a_{1}}{a_{0}}\right] R_{1}+\left[\frac{b_{0}}{a_{0}}+s \frac{b_{1}}{a_{0}}\right] R_{2}\right\rangle
$$

Então somente os valores $a_{1} a_{0}{ }^{-1}, b_{0} a_{0}{ }^{-1}$ e $b_{1} a_{0}{ }^{-1}$ precisam ser transmitidos, juntamente com um bit $w$ indicando se $a_{0}$ ou $b_{0}$ é inversível. Por fim, a chave pública passa a ser representada pela quíntupla $\left(a^{\prime}, w, a_{1} a_{0}{ }^{-1}, b_{0} a_{0}{ }^{-1}, b_{1} a_{0}{ }^{-1}\right) \in \mathbb{F}_{p^{2}} \times(\mathbb{Z} / 2 \mathbb{Z}) \times\left(\mathbb{Z} / 2^{m} \mathbb{Z}\right)^{3}$, que ocupa $2 \lg p+1+3 \cdot \frac{1}{2} \lg p \approx$ $3.5 \lg \mathbf{p}$ bits.

Contudo, as técnicas de compressão de chaves geram gargalos que impactam o desempenho do protocolo, conforme será abordado no próximo capítulo juntamente com propostas de melhorias. 


\section{Compressão eficiente de chaves isó- genas}

Descrevem-se nesse capítulo todas as técnicas propostas que, aplicadas em conjunto, melhoram o desempenho do processo de compressão de chaves isógenas. Vale ressaltar que as contribuições desta seção são individualmente pequenas, porém são todas complementares: o efeito de cada uma é cumulativo, levando a um resultado final substancial. Assim, este capítulo constitui, como um todo, o cerne de contribuições deste trabalho de pesquisa.

\subsection{Notação e convenções}

Todas as curvas são representadas usando o modelo Montgomery, a menos que especificado de outra forma.

A aritmética utilizada nos protocolos SIDH e SIKE se dá na extensão quadrática $\mathbb{F}_{p}^{2}$ do corpo base $\mathbb{F}_{p}$. O primo $p$ possui a forma $p:=2^{m} \cdot 3^{n}-1$, onde $m>2$ e $n>1$, de modo que $p \equiv 3(\bmod 4)$, o que garante que a aritmética ocorra de forma similar à dos números complexos. Sendo assim, a extensão quadrática $\mathbb{F}_{p^{2}} / \mathbb{F}_{p}$ é representada como $\mathbb{F}_{p^{2}}=\mathbb{F}_{p}(i)$, com $i^{2}+1=0$, e os elementos são expressos na forma $u+i v$, onde $u, v \in \mathbb{F}_{p}$.

Denota-se por i, c, m, s, e a os custos de inverter, elevar ao cubo, multiplicar, elevar ao quadrado e somar/subtrair/deslocar em $\mathbb{F}_{p}$, respectivamente, e por $\mathbf{I}, \mathbf{C}, \mathbf{M}, \mathbf{S}$, e $\mathbf{A}$ os custos das operações correspondentes em $\mathbb{F}_{p^{2}}$. O custo de mudar um sinal (por exemplo, ao lidar com o conjugado de um elemento do corpo) é desconsiderado. Os custos das operações em $\mathbb{F}_{p^{2}}$ em relação aos custos das operações em $\mathbb{F}_{p}$ podem ser aproximadas por: $1 \mathbf{I}=1 \mathbf{i}+2 \mathbf{m}+2 \mathbf{s}+1 \mathbf{a}$, $1 \mathbf{C}=2 \mathbf{m}+2 \mathbf{s}+5 \mathbf{a}, 1 \mathbf{M}=3 \mathbf{m}+5 \mathbf{a}, 1 \mathbf{S}=2 \mathbf{m}+3 \mathbf{a}$, e $1 \mathbf{A}=2 \mathbf{a}$, usando os análogos de corpos finitos das identidades trigonométricas de múltiplos ângulos de Viète (SPIEGEL; LIU, 1999, fórmulas 5.68 e 5.69).

\subsection{Decomposição reversa de bases}

Nesta seção, introduz-se a noção de decomposição reversa de bases para acelerar a compressão de chaves, tanto as de Alice quanto as de Bob, economizando o cálculo de um emparelhamento.

Posteriormente, na seção 4.3.1, mostra-se que, quando combinada com a geração de uma base emaranhada, esta técnica permite evitar duas multiplicações pelo cofator $3^{n}$ na compressão das chaves de Bob e uma multiplicação pelo mesmo cofator na descompressão de chave de Alice. 
Os resultados demonstram-se aqui do ponto de vista de Alice. As provas do ponto de vista de Bob são semelhantes.

A ideia principal adotada na literatura para obter compressão de chaves (AZARDERAKHSH et al., 2016; COSTELLO et al., 2017) é a seguinte: em vez de transmitir pontos $\phi_{\mathrm{A}}\left(P_{\mathrm{B}}\right), \phi_{\mathrm{A}}\left(Q_{\mathrm{B}}\right) \in E_{\mathrm{A}}\left[3^{n}\right]$, que são representados por duas abscissas em $\mathbb{F}_{p^{2}}$ e consomem $4 \log p$ bits, Alice calcula uma base "canônica" $R_{1}, R_{2} \in E_{\mathrm{A}}\left[3^{n}\right]$ e exprime a chave pública expandida nessa base como $\phi_{\mathrm{A}}\left(P_{\mathrm{B}}\right)=a_{0} R_{1}+b_{0} R_{2}$ e $\phi_{\mathrm{A}}\left(Q_{\mathrm{B}}\right)=a_{1} R_{1}+b_{1} R_{2}$. Em notação matricial,

$$
\left[\begin{array}{l}
\phi_{\mathrm{A}}\left(P_{\mathrm{B}}\right) \\
\phi_{\mathrm{A}}\left(Q_{\mathrm{B}}\right)
\end{array}\right]=\left[\begin{array}{ll}
a_{0} & b_{0} \\
a_{1} & b_{1}
\end{array}\right]\left[\begin{array}{l}
R_{1} \\
R_{2}
\end{array}\right] .
$$

Esta representação consiste em quatro inteiros muito menores $\left(a_{0}, b_{0}, a_{1}, b_{1}\right) \in\left(\mathbb{Z} / 3^{n} \mathbb{Z}\right)^{4}$ de tamanho total $2 \log p$ bits como sugerido em (AZARDERAKHSH et al., 2016). Isto foi refinado em (COSTELLO et al., 2017) pela transmissão apenas da tripla $\left(a_{0}^{-1} b_{0}, a_{0}^{-1} a_{1}, a_{0}^{-1} b_{1}\right) \in$ $\left(\mathbb{Z} / 3^{n} \mathbb{Z}\right)^{3}$ ou $\left(b_{0}^{-1} a_{0}, b_{0}^{-1} a_{1}, b_{0}^{-1} b_{1}\right) \in\left(\mathbb{Z} / 3^{n} \mathbb{Z}\right)^{3}$ dependendo de se $a_{0}$ ou $b_{0}$ é inversível. Logo, apenas (3/2) $\log p$ mais um bit indicando a inversibilidade de $a_{0}$ ou $b_{0}$ módulo $3^{n}$ são necessários. Nas técnicas acima, os coeficientes $a_{0}, b_{0}, a_{1}, b_{1}$ podem ser calculados com cinco emparelhamentos de Tate dados por

$$
\begin{aligned}
& g_{0}=\mathrm{e}_{3^{n}}\left(R_{1}, R_{2}\right) \\
& g_{1}=\mathrm{e}_{3^{n}}\left(R_{1}, \phi_{\mathrm{A}}\left(P_{\mathrm{B}}\right)\right)=\mathrm{e}_{3^{n}}\left(R_{1}, a_{0} R_{1}+b_{0} R_{2}\right)=g_{0}^{b_{0}} \\
& g_{2}=\mathrm{e}_{3^{n}}\left(R_{1}, \phi_{\mathrm{A}}\left(Q_{\mathrm{B}}\right)\right)=\mathrm{e}_{3^{n}}\left(R_{1}, a_{1} R_{1}+b_{1} R_{2}\right)=g_{0}^{b_{1}} \\
& g_{3}=\mathrm{e}_{3^{n}}\left(R_{2}, \phi_{\mathrm{A}}\left(P_{\mathrm{B}}\right)\right)=\mathrm{e}_{3^{n}}\left(R_{2}, a_{0} R_{1}+b_{0} R_{2}\right)=g_{0}^{-a_{0}} \\
& g_{4}=\mathrm{e}_{3^{n}}\left(R_{2}, \phi_{\mathrm{A}}\left(Q_{\mathrm{B}}\right)\right)=\mathrm{e}_{3^{n}}\left(R_{2}, a_{1} R_{1}+b_{1} R_{2}\right)=g_{0}^{-a_{1}} .
\end{aligned}
$$

A partir disso, Alice pode recuperar $a_{0}, b_{0}, a_{1}$ e $b_{1}$ resolvendo logaritmos discretos num subgrupo multiplicativo de ordem suave $3^{n}$ usando o algoritmo Pohlig-Hellman (POHLIG; HELLMAN, 1978).

Uma vez que $\phi_{\mathrm{A}}\left(P_{\mathrm{B}}\right)$ e $\phi_{\mathrm{A}}\left(Q_{\mathrm{B}}\right)$ também formam uma base de $E_{\mathrm{A}}\left[3^{n}\right]$, a matriz dos coeficientes de (4.1) é inversível módulo $3^{n}$. Portanto pode-se escrever

$$
\left[\begin{array}{l}
R_{1} \\
R_{2}
\end{array}\right]=\left[\begin{array}{ll}
c_{0} & d_{0} \\
c_{1} & d_{1}
\end{array}\right]\left[\begin{array}{l}
\phi_{\mathrm{A}}\left(P_{\mathrm{B}}\right) \\
\phi_{\mathrm{A}}\left(Q_{\mathrm{B}}\right)
\end{array}\right]
$$

invertendo a matriz em (4.1). Mudando os papéis das bases $\left\{R_{1}, R_{2}\right\}$ e $\left\{\phi_{\mathrm{A}}\left(P_{\mathrm{B}}\right), \phi_{\mathrm{A}}\left(Q_{\mathrm{B}}\right)\right\}$ 
em (4.2) obtém-se

$$
\begin{aligned}
h_{0} & =\mathrm{e}_{3^{n}}\left(\phi_{\mathrm{A}}\left(P_{\mathrm{B}}\right), \phi_{\mathrm{A}}\left(Q_{\mathrm{B}}\right)\right) \\
h_{1} & =\mathrm{e}_{3^{n}}\left(\phi_{\mathrm{A}}\left(P_{\mathrm{B}}\right), R_{1}\right) \\
& =\mathrm{e}_{3^{n}}\left(\phi_{\mathrm{A}}\left(P_{\mathrm{B}}\right), c_{0} \phi_{\mathrm{A}}\left(P_{\mathrm{B}}\right)+d_{0} \phi_{\mathrm{A}}\left(Q_{\mathrm{B}}\right)\right)=h_{0}^{d_{0}} \\
h_{2} & =\mathrm{e}_{3^{n}}\left(\phi_{\mathrm{A}}\left(P_{\mathrm{B}}\right), R_{2}\right) \\
& =\mathrm{e}_{3^{n}}\left(\phi_{\mathrm{A}}\left(P_{\mathrm{B}}\right), c_{1} \phi_{\mathrm{A}}\left(P_{\mathrm{B}}\right)+d_{1} \phi_{\mathrm{A}}\left(Q_{\mathrm{B}}\right)\right)=h_{0}^{d_{1}} \\
h_{3} & =\mathrm{e}_{3^{n}}\left(\phi_{\mathrm{A}}\left(Q_{\mathrm{B}}\right), R_{1}\right) \\
& =\mathrm{e}_{3^{n}}\left(\phi_{\mathrm{A}}\left(Q_{\mathrm{B}}\right), c_{0} \phi_{\mathrm{A}}\left(P_{\mathrm{B}}\right)+d_{0} \phi_{\mathrm{A}}\left(Q_{\mathrm{B}}\right)\right)=h_{0}^{-c_{0}} \\
h_{4} & =\mathrm{e}_{3^{n}}\left(\phi_{\mathrm{A}}\left(Q_{\mathrm{B}}\right), R_{2}\right) \\
& =\mathrm{e}_{3^{n}}\left(\phi_{\mathrm{A}}\left(Q_{\mathrm{B}}\right), c_{1} \phi_{\mathrm{A}}\left(P_{\mathrm{B}}\right)+d_{1} \phi_{\mathrm{A}}\left(Q_{\mathrm{B}}\right)\right)=h_{0}^{-c_{1}} .
\end{aligned}
$$

O primeiro emparelhamento em (4.4) calcula-se como

$$
h_{0}=\mathrm{e}_{3^{n}}\left(P_{\mathrm{B}}, \hat{\phi}_{\mathrm{A}} \circ \phi_{\mathrm{A}}\left(Q_{\mathrm{B}}\right)\right)=\mathrm{e}_{3^{n}}\left(P_{\mathrm{B}},\left[\operatorname{deg} \phi_{\mathrm{A}}\right] Q_{\mathrm{B}}\right)=\mathrm{e}_{3^{n}}\left(P_{\mathrm{B}}, Q_{\mathrm{B}}\right)^{2^{m}}
$$

que depende apenas dos parâmetros públicos $P_{\mathrm{B}}, Q_{\mathrm{B}}$ e $m$. Logo, ele pode ser calculado de uma vez por todas e incluído nesses parâmetros públicos. Em particular, somente os emparelhamentos $h_{1}, h_{2}, h_{3}$ e $h_{4}$ precisam ser calculados em tempo de execução. Os logaritmos discretos são computados como antes via Pohlig-Hellman, resultando em $c_{0}=-\log _{h_{0}} h_{3}, d_{0}=\log _{h_{0}} h_{1}, c_{1}=$ $-\log _{h_{0}} h_{4}$ e $d_{1}=\log _{h_{0}} h_{2}$. A seguir, Alice inverte a matriz de coeficientes de (4.3) para obter a matriz de coeficientes (4.1). Explicitamente,

$$
\left[\begin{array}{ll}
a_{0} & b_{0} \\
a_{1} & b_{1}
\end{array}\right]=\frac{1}{D}\left[\begin{array}{cc}
d_{1} & -d_{0} \\
-c_{1} & c_{0}
\end{array}\right]
$$

onde $D=c_{0} d_{1}-c_{1} d_{0}$. Note que a inversão adicional de $D^{-1}$ não precisa ser efetuada quando se adota a técnica em (COSTELLO et al., 2017). Mais precisamente, uma vez que pelo menos um dentre $d_{0}$ ou $d_{1}$, por exemplo $d_{1}$, é inversível módulo $3^{n}$, Alice transmite a tupla

$$
\begin{aligned}
& \left(a_{0}^{-1} b_{0}, a_{0}^{-1} a_{1}, a_{0}^{-1} b_{1}\right) \\
& =\left(-d_{1}^{-1} D D^{-1} d_{0},-d_{1}^{-1} D D^{-1} c_{1}, d_{1}^{-1} D D^{-1} c_{0}\right) \\
& =\left(-d_{1}^{-1} d_{0},-d_{1}^{-1} c_{1}, d_{1}^{-1} c_{0}\right)
\end{aligned}
$$

que é independente de $D$.

\subsection{Geração de bases emaranhadas}

Introduz-se agora uma técnica para criar uma base completa do grupo de $2^{m}$-torção a partir de um único ponto específico de ordem $2^{m}$. Em outras palavras, o custo envolvido é essencialmente o de calcular um gerador para um único subgrupo de ordem $2^{m}$ em $E\left[2^{m}\right]$ : um gerador para o subgrupo linearmente independente torna-se imediatamente disponível quase 
sem custo computacional. Consequentemente, o teste de independência linear, que consiste de duas multiplicações escalares por $2^{m-1}$, pode ser completamente evitado. Isto é semelhante aos mapas de distorção, embora nenhum esteja normalmente disponível para as curvas envolvidas no SIDH. As bases resultantes são aqui denominadas "emaranhadas" por analogia com o fenômeno quântico onde as propriedades de uma entidade são inteiramente determinadas pelas propriedades de outra entidade, a despeito da separação entre elas. ${ }^{1}$

Para construir uma base emaranhada $\langle P, Q\rangle=E\left[2^{m}\right]$ para $E: y^{2}=x^{3}+A x^{2}+x$, de certa forma "subvertem-se" as fórmulas originais Elligator 2 (BERNSTEIN et al., 2013) por um motivo diferente de codificar pontos na forma de cadeias aleatórias de bits, a saber, obter dois pontos linearmente independente em $E$ de uma só vez. No que se segue, o valor $t:=u_{0} r$, com $u_{0} \in \mathbb{F}_{p^{2}} \backslash \mathbb{F}_{p}$ e $r \in \mathbb{F}_{p}^{*}$ tais que $u:=u_{0}^{2} \in \mathbb{F}_{p^{2}} \backslash \mathbb{F}_{p}$, denota um resíduo quadrático em vez de um não-resíduo como no Elligator 2 original. O teorema 30 estabelece as propriedades desta nova construção.

Observação. Da mesma maneira que em (BERNSTEIN et al., 2013), assume-se aqui que $A \neq 0$ no modelo de Montgomery. $\mathrm{O}$ caso $A=0$ corresponde à curva $E_{0}: y^{2}=x^{3}+x$ usada como curva inicial na maioria das implementações. Isto não representa problema no contexto presente porque, em primeiro lugar, não há geração de base em tempo de execução para a curva inicial $E$, e em segundo, a probabilidade de encontrar $E_{0}$ no meio do acordo de chaves é desprezível (em geral, evitam-se no protocolo curvas $E / \mathbb{F}_{p}$ definidas sobre o corpo base: se isso acontecer, a praxe seria recomeçar o protocolo para garantir que $E$ esteja definida propriamente sobre $\mathbb{F}_{p^{2}}$, mas a chance de um recomeço desta natureza ocorrer é apenas cerca de $1 / p$ ). Alice e Bob podem evitar esta circunstância verificando o j-invariante de suas chaves públicas.

Teorema 30. Dada uma curva elíptica supersingular de Montgomery $E_{\mathrm{A}} / \mathbb{F}_{p^{2}}: y^{2}=x\left(x^{2}+A x+1\right)$ onde $p=2^{m} \cdot 3^{n}-1, \# E_{\mathrm{A}}\left(\mathbb{F}_{p^{2}}\right)=(p+1)^{2}$, e $A \neq 0$, seja $t \in \mathbb{F}_{p^{2}}$ um elemento tal que $t^{2} \in \mathbb{F}_{p^{2}} \backslash \mathbb{F}_{p}$, e seja $x_{1}:=-A /\left(1+t^{2}\right)$ um não-resíduo quadrático que define a abscissa de um ponto $P_{1} \in E_{\mathrm{A}}\left(\mathbb{F}_{p^{2}}\right)$. Então $x_{2}:=-x_{1}-A$ define a abscissa de outro ponto $P_{2} \in E_{\mathrm{A}}\left(\mathbb{F}_{p^{2}}\right)$ tal que $\left\langle[h] P_{1},[h] P_{2}\right\rangle=E_{\mathrm{A}}\left[2^{m}\right]$, onde $h:=3^{n}$ é o cofator do grupo de $2^{m}$-torção.

Demonstração. Uma vez que $x_{2}=t^{2} x_{1}$, ambas as abscissas são não-resíduos quadráticos, e devido a (HUSEMÖLLER, 2004, Capítulo 1 (§4), Teorema 4.1) os dois pontos $P_{1}=\left(x_{1}, y_{1}\right)$, $P_{2}=\left(t^{2} x_{1}, t y_{1}\right), \operatorname{com} x_{1}+t^{2} x_{1}+A=0$, não estão em [2] $E_{\mathrm{A}}$. Logo, os pontos $[h] P_{1}$ e $[h] P_{2}$ são pontos da $2^{m}$-torção completa. Para provar que $h \cdot P_{1}, h \cdot P_{2}$ geram $E_{\mathrm{A}}\left[2^{m}\right]$, é necessário provar que $\left[h \cdot 2^{m-1}\right]\left(P_{1}-P_{2}\right) \neq 0$, ou equivalentemente que $(u, v)=P_{1}+\left(-P_{2}\right) \notin[2] E_{\mathrm{A}}$. Pela lei de

1 Ressalta-se, no entanto, que aqui a nomenclatura é meramente análoga: não existe processo quântico envolvido na construção. 
adição (SILVERMAN, 1986, Algoritmo 2.3) em $E_{\mathrm{A}}$ obtém-se:

$$
\begin{aligned}
& \lambda=\frac{y_{2}-y_{1}}{x_{2}-x_{1}}=\frac{-t y_{1}-y_{1}}{t^{2} x_{1}-x_{1}}=\frac{-(t+1) y_{1}}{\left(t^{2}-1\right) x_{1}}=\frac{-y_{1}}{(t-1) x_{1}}, \\
& \mu=\frac{y_{1} x_{2}-y_{2} x_{1}}{x_{2}-x_{1}}=\frac{t(t+1) y_{1} x_{1}}{(t+1)(t-1) x_{1}}=-\lambda t x_{1}, \\
& u=\lambda^{2}-A-x_{1}-x_{2}=\lambda^{2}, \\
& v=-\lambda u-\mu=-\lambda u-\left(-\lambda t x_{1}\right)=-\lambda\left(u-t x_{1}\right) .
\end{aligned}
$$

Das identidades acima, vê-se que $v^{2}=\lambda^{2}\left(u-t x_{1}\right)^{2}=u\left(u^{2}+A u+1\right)$ e portanto $u^{2}+A u+1=\left(u-t x_{1}\right)^{2}$. Seja $w:=u-t x_{1}=\sqrt{u^{2}+A u+1}$. Então $1-(u-w)^{2}=1-t^{2} x_{1}^{2}=x_{1}^{2}+A x_{1}+1$, que é um não-resíduo quadrático porque $x_{1}$ é ele mesmo um não-resíduo quadrático, enquanto que o seu produto é obviamente um quadrado, $x_{1}\left(x_{1}^{2}+A x_{1}+1\right)=y_{1}^{2}$, em virtude da equação da curva. Um cálculo direto mostra que $\left(1-(u+w)^{2}\right)\left(1-(u-w)^{2}\right)=u^{2}\left(A^{2}-4\right)$. Mas $A^{2}-4$ é um resíduo quadrático porque $E_{\mathrm{A}}$ contém todo o grupo de 2-torção sobre $\mathbb{F}_{p^{2}}$. Portanto, ambos os valores $(u \pm w)^{2}-1$ têm o mesmo caráter quadrático, isto é, ambos são não-resíduos quadráticos pelo raciocínio acima.

Agora $^{2}$ assuma por absurdo que $P_{1}-P_{2} \in[2] E_{\mathrm{A}}$, ou seja, que exista um ponto $(x, y) \in$ $E_{\mathrm{A}}\left(\mathbb{F}_{p^{2}}\right)$ tal que [2] $(x, y)=(u, v)$. A partir da fórmula de duplicação de ponto em $E_{\mathrm{A}}$ obtém-se:

$$
u=\frac{\left(x^{2}-1\right)^{2}}{4 x\left(x^{2}+A x+1\right)} .
$$

Isto leva à equação de quarto grau $\left(x^{2}-1\right)^{2}-4 u x\left(x^{2}+A x+1\right)=0$. Uma vez que $x \neq 0$, podem-se dividir ambos os lados por $x^{2}$ e rearranjar alguns termos para obter:

$$
\left(x+\frac{1}{x}\right)^{2}-4 u\left(x+\frac{1}{x}\right)-4(A u+1)=0 .
$$

Resolvendo esta equação quadrática, obtém-se:

$$
x+\frac{1}{x}=\frac{4 u \pm \sqrt{16\left(u^{2}+A u+1\right)}}{2}=\frac{4 u \pm 4 w}{2}=2(u \pm w) .
$$

Por sua vez, isto leva a uma segunda equação quadrática $x^{2}-2(u \pm w) x+1=0$. Como $x \in \mathbb{F}_{p^{2}}$ mais uma vez, o discriminante $4(u \pm w)^{2}-4$ e portanto pelo menos um dentre $(u \pm w)^{2}-1$ deve ser um resíduo quadrático. Mas isto contradiz a observação prévia de que $(u \pm w)^{2}-1$ são ambos não-resíduos quadráticos. $\operatorname{Logo}, P_{1}-P_{2} \notin[2] E$, confirmando que $\left\langle[h] P_{1},[h] P_{2}\right\rangle=E_{\mathrm{A}}\left[2^{m}\right]$.

Na prática, pode-se implementar eficientemente a geração de base emaranhada da seguinte maneira. Seja $u_{0} \in \mathbb{F}_{p^{2}} \backslash \mathbb{F}_{p}$ tal que $u:=u_{0}^{2} \in \mathbb{F}_{p^{2}} \backslash \mathbb{F}_{p}$, por exemplo, $u_{0}=1+i$ e $u=2 i$. Defina duas tabelas de pares $(r, v) \operatorname{com} v:=1 /\left(1+u r^{2}\right)$ :

- a tabela $T_{1}$ contém pares $(r, v)$ onde $v$ é um não-resíduo quadrático,

\footnotetext{
2 Esta parte segue de perto a ideia de (HUSEMÖLLER, 2004, Capítulo 1 (§4), Teorema 4.1).
} 
- a tabela $T_{2}$ contém pares $(r, v)$ onde $v$ é um resíduo quadrático.

Fazendo um teste de quadraticidade em $A$ uma única vez por curva e restringindo a busca à tabela com quadraticidade oposta garante que $x:=-A v$ é um não-resíduo quadrático. Repetindo testes de quadraticidade até garantir que uma ordenada $y$ correspondente à abscissa $x$ existe, $\mathrm{e}$ completando uma extração de raiz quadrada em $\mathbb{F}_{p^{2}}$ para obter esse $y$, produzem-se de uma vez 2 pontos cujas ordens são múltiplos de $2^{m}$. Este processo é exposto em detalhes no Algoritmo 2.

A vantagem da técnica de base emaranhada fica clara quando se compara aos métodos prévios de geração de base, conforme Costello et al. (COSTELLO et al., 2017).

Base emaranhada: determinar o caráter quadrático de $A$ custa $(m+n+1) \mathbf{s}+n \mathbf{m}$. O laço principal roda duas vezes em média, com um custo $2(m+n+1) \mathbf{s}+(2 n+22) \mathbf{m}$. O último estágio é completar a raiz quadrada e custa $(m+n-1) \mathbf{s}+(n+1) \mathbf{m}+1 \mathbf{i}$. O custo total do algoritmo é então

$$
(4 m+4 n+2) \mathbf{s}+(4 n+23) \mathbf{m}+1 \mathbf{i} .
$$

Base comum: calcular a abscissa de um ponto da curva custa $(2 n+22) \mathbf{m}+2(m+n+1) \mathbf{s}$. Multiplicar pelo cofator $3^{n}$ requer $n$ triplicações de ponto a um custo $32 n \mathbf{m}$. O teste de independência linear requer $m-1$ duplicações de ponto a um custo $16(m-1) \mathbf{m}$. Logo, obter o primeiro ponto da base custa $(34 n+16 m+6) \mathbf{m}+2(m+n+1) \mathbf{s}$. O segundo ponto obtém-se da mesma maneira, exceto que também incorre o teste de independência linear. Isto se faz num laço que roda duas vezes em média. $\mathrm{O}$ custo esperado de obter o segundo ponto é, portanto, o dobro do custo de obter o primeiro ponto incluindo o passo de $m-1$ duplicações.O último estágio do algoritmo é recuperar as ordenadas y dos pontos, o que custa $(4 m+4 n) \mathbf{s}+(4 n+36) \mathbf{m}+2 \mathbf{i}$. Somando todas essas contribuições, o custo total do algoritmo é

$$
(10 m+10 n+6) \mathbf{s}+(48 m+106 n+54) \mathbf{m}+2 \mathbf{i} .
$$

Para os valores $m=372$ e $n=239$ previamente abordados na literatura, e assumindo $\mathbf{s}=0.8 \mathbf{m} \mathrm{e}$ $\mathbf{i}=100 \mathbf{m}$, obtém-se uma razão entre o desempenho das bases emaranhadas em relação às bases comuns de 15.92.

\subsubsection{Evitando a multiplicação por cofator}

Combinando decomposição reversa de bases e geração de base emaranhada, é possível evitar mais duas multiplicações pelo grande cofator $3^{n}$ durante a compressão da chave pública de Bob e uma durante a descompressão da chave pública de Alice. Para isso, note-se primeiro que o Algoritmo 2 já incorpora essa otimização, isto é, os pontos devolvidos $S_{1}$ e $S_{2}$ satisfazem $\left(\left[3^{n}\right] S_{1},\left[3^{n}\right] S_{2}\right)=\left(R_{1}, R_{2}\right)$ tais que $\left\langle R_{1}, R_{2}\right\rangle=E\left[2^{m}\right]$. Isto só é possível porque, na decomposição reversa de base, os emparelhamentos de Tate $h_{i}$ recebem os pontos $S_{i}$ como seu segundo 


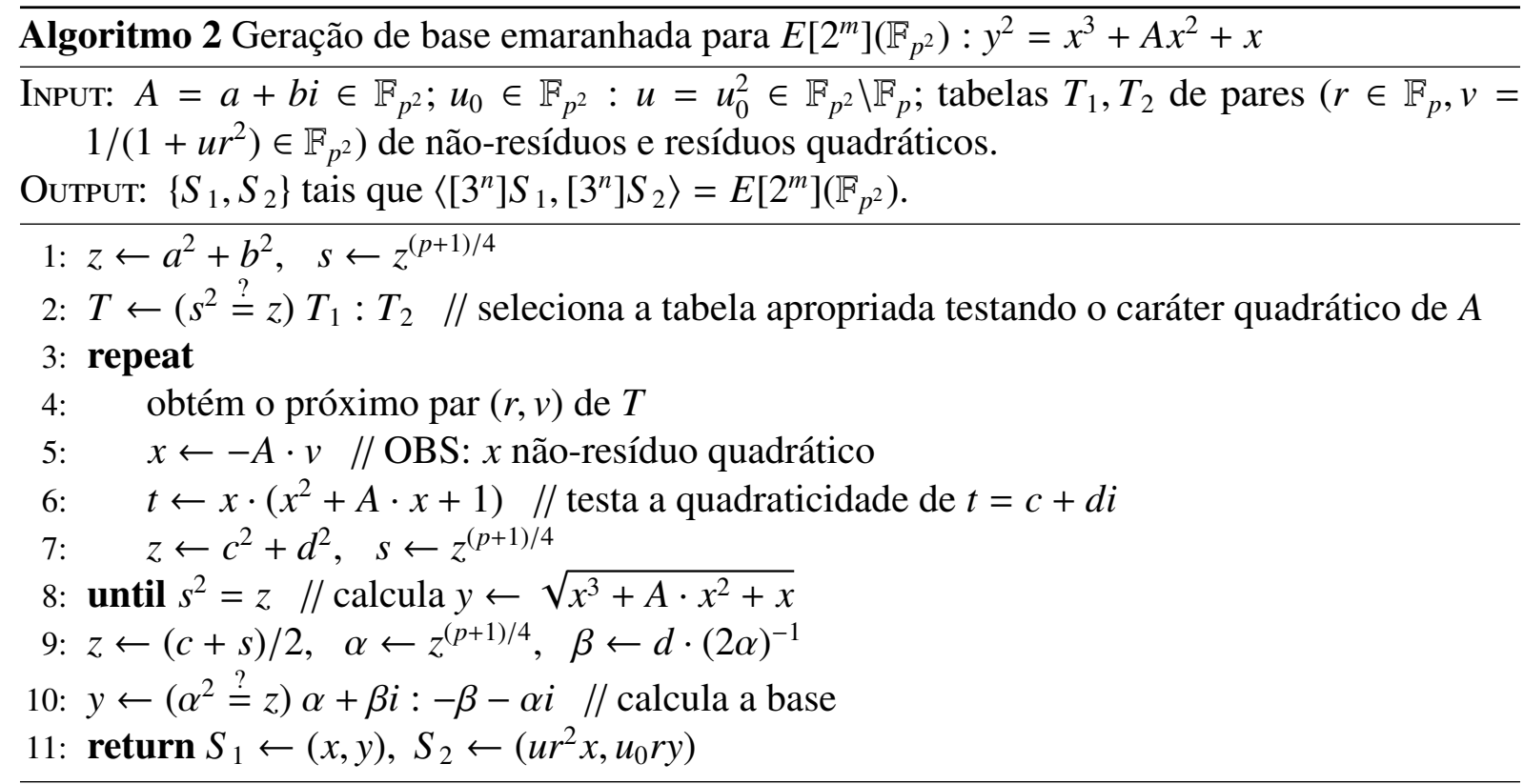

argumento, que não precisa ser necessariamente reduzido pelo cofator. Nesse caso, para $R_{1}=$ $c_{0} \phi_{\mathrm{B}}\left(P_{\mathrm{A}}\right)+d_{0} \phi_{\mathrm{B}}\left(Q_{\mathrm{A}}\right)$ e $R_{2}=c_{1} \phi_{\mathrm{B}}\left(P_{\mathrm{A}}\right)+d_{1} \phi_{\mathrm{B}}\left(Q_{\mathrm{A}}\right)$, os respectivos cálculos de emparelhamentos são:

$$
\begin{aligned}
k_{0} & =\mathrm{e}_{2^{m}}\left(\phi_{\mathrm{B}}\left(P_{\mathrm{A}}\right), \phi_{\mathrm{B}}\left(Q_{\mathrm{A}}\right)\right) \\
k_{1} & =\mathrm{e}_{2^{m}}\left(\phi_{\mathrm{B}}\left(P_{\mathrm{A}}\right), S_{1}\right) \\
& =\mathrm{e}_{2^{m}}\left(\phi_{\mathrm{B}}\left(P_{\mathrm{A}}\right),\left[3^{-n}\right] R_{1}\right)=k_{0}^{3^{-n} d_{0}} \\
k_{2} & =\mathrm{e}_{2^{m}}\left(\phi_{\mathrm{B}}\left(P_{\mathrm{A}}\right), S_{2}\right) \\
& =\mathrm{e}_{2^{m}}\left(\phi_{\mathrm{B}}\left(P_{\mathrm{A}}\right),\left[3^{-n}\right] R_{2}\right)=k_{0}^{3^{-n} d_{1}} \\
k_{3} & =\mathrm{e}_{2^{m}}\left(\phi_{\mathrm{B}}\left(Q_{\mathrm{A}}\right), S_{1}\right) \\
& =\mathrm{e}_{2^{m}}\left(\phi_{\mathrm{B}}\left(Q_{\mathrm{A}}\right),\left[3^{-n}\right] R_{1}\right)=k_{0}^{-3^{-n} c_{0}} \\
k_{4} & =\mathrm{e}_{2^{m}}\left(\phi_{\mathrm{B}}\left(Q_{\mathrm{A}}\right), S_{2}\right) \\
& =\mathrm{e}_{2^{m}}\left(\phi_{\mathrm{B}}\left(Q_{\mathrm{A}}\right),\left[3^{-n}\right] R_{2}\right)=h_{0}^{-3^{-n} c_{1}} .
\end{aligned}
$$

Assim, os logaritmos discretos são os desejados a menos de um fator $3^{-n}$, e dados por $\hat{c}_{0}=$ $-\log _{k_{0}} k_{3}=3^{-n} c_{0}, \hat{d}_{0}=\log _{k_{0}} k_{1}=3^{-n} d_{0}, \hat{c}_{1}=-\log _{k_{0}} k_{4}=3^{-n} c_{1}$, e $\hat{d}_{1}=\log _{k_{0}} k_{2}=3^{-n} d_{1}$. Note que $3^{-n} \bmod 2^{m}$ deve ser ímpar, o que implica que $\hat{c}_{0}$ ou $\hat{d}_{0}$ é inversível se, e somente se, $c_{0}$ ou $d_{0}$ é inversível. De maneira similar à situação na Seção 4.2, ao usar a compressão com apenas 3 coefficients como em (COSTELLO et al., 2017) Bob transmite exatamente os coeficientes originais. Assumindo que $\hat{c}_{0}$ seja inversível, então:

$$
\begin{aligned}
& \left(\hat{c}_{0}^{-1} \hat{d}_{0}, \hat{c}_{0}^{-1} \hat{c}_{1}, \hat{c}_{0}^{-1} \hat{d}_{1}\right) \\
& =\left(c_{0}^{-1} 3^{n} 3^{-n} d_{0}, c_{0}^{-1} 3^{n} 3^{-n} c_{1}, c_{0}^{-1} 3^{n} 3^{-n} d_{1}\right) \\
& =\left(c_{0}^{-1} d_{0}, c_{0}^{-1} c_{1}, c_{0}^{-1} d_{1}\right)
\end{aligned}
$$

A derivação quando $d_{0}$ é inversível é análoga.

Para descomprimir a chave pública de Bob, Alice precisa realizar uma única multiplicação pelo cofator $3^{n}$ como segue. Assuma que $a_{0}$ seja inversível módulo $2^{m}$ de modo que Alice recebe 
a tripla $\left(a_{0}^{-1} b_{0}, a_{0}^{-1} a_{1}, a_{0}^{-1} b_{1}\right)$. Ela precisa calcular o núcleo $\operatorname{ker}\left(\phi_{\mathrm{AB}}\right)=\left\langle\phi_{\mathrm{B}}\left(P_{\mathrm{A}}\right)+s k_{\mathrm{A}} \cdot \phi_{\mathrm{B}}\left(Q_{\mathrm{A}}\right)\right\rangle$, que pode ser escrito como $\left\langle a_{0} R_{1}+b_{0} R_{2}+s k_{\mathrm{A}} \cdot\left(a_{1} R_{1}+b_{1} R_{2}\right)\right\rangle=\left\langle\left(a_{0}+s k_{\mathrm{A}} a_{1}\right) R_{1}+\left(b_{0}+s k_{\mathrm{A}} b_{1}\right) R_{2}\right\rangle$. Conforme ressaltado em (COSTELLO et al., 2017), calcula-se $\operatorname{ker}\left(\phi_{\mathrm{AB}}\right) \operatorname{como} a_{0}^{-1} \operatorname{ker}\left(\phi_{\mathrm{AB}}\right)=$ $\left\langle\left(1+s k_{\mathrm{A}} a_{0}^{-1} a_{1}\right) R_{1}+\left(a_{0}^{-1} b_{0}+s k_{\mathrm{A}} a_{0}^{-1} b_{1}\right) R_{2}\right\rangle$, que pode ser feito com uma scalar multiplicação e uma adição de ponto escrevendo $\operatorname{ker}\left(\phi_{\mathrm{AB}}\right)=\left\langle R_{1}+\left(1+s k_{\mathrm{A}} a_{0}^{-1} a_{1}\right)^{-1}\left(a_{0}^{-1} b_{0}+s k_{\mathrm{A}} a_{0}^{-1} b_{1}\right) R_{2}\right\rangle$. Se Alice usar o Algoritmo 2, ela obterá uma base emaranhada $\left\{S_{1}, S_{2}\right\}$ tal que $\left(R_{1}, R_{2}\right)=\left(\left[3^{n}\right] S_{1},\left[3^{n}\right] S_{2}\right)$. Desta maneira, ela poderá calcular $T=\left\langle S_{1}+\left(1+s k_{\mathrm{A}} a_{0}^{-1} a_{1}\right)^{-1}\left(a_{0}^{-1} b_{0}+s k_{\mathrm{A}} a_{0}^{-1} b_{1}\right) S_{2}\right.$ primeiro e então recuperar o núcleo correto $\operatorname{ker}\left(\phi_{\mathrm{AB}}\right)=\left\langle\left[3^{n}\right] T\right\rangle$ fazendo uma multiplicação escalar pelo cofator.

\subsection{Geração de bases em $E\left[3^{n}\right]$}

A abordagem de base emaranhada introduzida na Seção 4.3 não pode ser generalizada de forma direta para o caso ternário. Não há uma maneira clara de escolher simultaneamente dois pontos linearmente independentes. Como consequência, adota-se a abordagem ingênua de escolher pontos candidatos aleatoriamente e testá-los quanto à ordem correta e independência linear para gerar bases para $E\left[3^{n}\right]$.

A técnica de $\ell$-descenso de Schaefer-Stoll (SCHAEFER; STOLL, 2004) identifica um ponto de $n$-torção no conjunto $[\ell] E$ de uma curva quando $\ell \mid n$. Em outras palavras, ela determina eficientemente se um ponto $P \in E[n]$ está propriamente na torção menor $E[n / \ell]$. Isso filtra pontos inadequados para formar uma base da $n$-torção completa. Contudo, a técnica exige o conhecimento de um ponto de $\ell$-torção, uma vez que consiste em aplicar a equação da reta tangente à curva num ponto de $\ell$-torção ao ponto $P$ e testar se o resultado é uma resíduo de $\operatorname{ordem} \ell$ (uma raiz $\ell$-ésima no corpo finito). Numa curva de Montgomery, um ponto de ordem 2 é sempre imediatamente disponível: é o ponto $(0,0)$. Por isso, aplicar a técnica de 2-descenso é simples. Já um ponto de ordem 3 ou superior tipicamente não é simples de determinar.

Costello et al. sugerem precisamente o uso do 3-descenso de Schaefer e Stoll e afirmam ganhos significativos. No entanto, não foi possível reproduzir e, portanto, verificar suas reivindicações. Pelo contrário, observou-se que a abordagem ingênua é sempre mais rápida quando comparada com o 3 -descenso, com uma relação de custo $\mathcal{C}_{\text {ingênua }} / \mathcal{C}_{3 \text {-descenso }} \approx 0.89$, contrária a afirmação anterior. A seguinte análise detalhada parece corroborar a relação de custo observada.

$\mathrm{Na}$ abordagem de 3-descenso de Costello et al., as reivindicações de ganho se aplicam somente para testar se um ponto pertence a $E \backslash[3] E$. Nota-se que o custo de gerar candidatos também deve ser levado em consideração.

Assim, por um lado, o teste da abordagem ingênua envolve:

- uma construção Elligator com custo $\mathbf{L}$ por tentativa;

- $m$ duplicações ao custo $\mathbf{D}$ cada, por tentativa; 
- $n$ - 1 triplicações ao custo $\mathbf{T}$ cada, por tentativa,

- 9/8 tentativas na média com custo $\mathbf{P}$ cada, para obter o ponto de ordem certa;

- 4/3 construções e verificações de ponto a um custo médio de $\mathbf{C}$ cada, para obter um segundo ponto de linearmente independente.

Portanto, o custo ingênuo de se obter a coordenada $x$ dos pontos da base é $(1+4 / 3)(9 / 8) \mathbf{P}+$ (4/3)C, somado ao custo individual $\mathbf{E}$ para completar a coordenada do ponto através do cálculo de duas soluções da equação, ou $(1+4 / 3)(9 / 8) \mathbf{P}+(4 / 3) \mathbf{C}+2 \mathbf{E}$ global.

Estimando $\mathbf{L}=(0.8 m+1.8 n+9.8) \mathbf{m}+20 \mathbf{a}, \mathbf{D}=13 \mathbf{m}+29 \mathbf{a}, \mathbf{T}=27 \mathbf{m}+61 \mathbf{a}$, $\mathbf{C}=2(3 \mathbf{m}+5 \mathbf{a}), \mathbf{E}=1 \mathbf{i}+(1.6 m+3.6 n+27.6) \mathbf{m}+46 \mathbf{a}$, e notando que $\mathbf{P}=\mathbf{L}+m \mathbf{D}+(n-1) \mathbf{T}$, conclui-se que o custo da abordagem ingênua é de $C_{\text {ingênua }} \approx 2 \mathbf{i}+(39.425 m+82.8 n+18.05) \mathbf{m}+$ $(76.125 m+160.125 n-3.708) \mathbf{a}$.

Por outro lado, a abordagem usando 3-descenso envolve inicialmente:

- uma construção Elligator com custo $\mathbf{L}$;

- $m$ duplicações ao custo $\mathbf{D}$;

- $n-1$ triplicações ao custo $\mathbf{T}$;

- uma solução da equação da curva a um custo de $\mathbf{E}$;

- uma construção da função de filtro a um custo de $\mathbf{F}$ para obter um ponto $P_{3}$ de ordem 3 e possivelmente ser o primeiro ponto da base.

Observe que uma fórmula de duplicação mais cara a um custo $\mathbf{D}^{\prime}=16 \mathbf{m}+34 \mathbf{a}(\mathrm{em}$ vez de $\mathbf{D}=13 \mathbf{m}+29 \mathbf{a}$ ) foi empregada por (COSTELLO et al., 2017) que usa os coeficientes da curva na forma projetiva. Esta fórmula projetiva é útil para calcular $2^{m}$-isogenias projetivas, mas nota-se que não é necessária no contexto da geração da base e pode-se simplesmente manter a duplicação de Montgomery mais eficiente usual (MONTGOMERY, 1987). Considera-se a versão mais rápida nas estimativas.

O método de 3-descenso exige (com probabilidade 1/9) uma construção de ponto filtrado a um custo $\mathbf{Z}$ para obter o primeiro ponto da base, mais $4 / 3$ construções de pontos filtrados e verificações na média (já que a probabilidade de sucesso na verificação é de 3/4) para obter o segundo ponto da base e, finalmente, duas soluções da equação da curva.

Estimando $\mathbf{F}=1 \mathbf{i}+12.6 \mathbf{m}+29 \mathbf{a}, \mathbf{Z}=3 \mathbf{i}+(17.8 m+37.8 n+39.3) \mathbf{m}+(29 m+61 n+57.5) \mathbf{a}$, e mantendo as mesmas estimativas restantes como antes, conclui-se que o custo do 3-descenso é $C_{3 \text {-descenso }}:=(3(4 / 3)+3(1 / 9)+4) \mathbf{i}+((17.8 m+37.8 n+45.3)(4 / 3)+(17.8 m+37.8 n+39.3)(1 / 9)+$ $31.2 m+39.6 n+78.2) \mathbf{m}+((29 m+61 n+67.5)(4 / 3)+(29 m+61 n+57.5)(1 / 9)+29 m+61 n+126) \mathbf{a}$. 
As estimativas produzem uma relação de custo $C_{\text {ingênua }} / C_{3 \text {-descenso }} \approx 0.89$, que bate com o observado experimentalmente. Isso vai contra as reivindicações de (COSTELLO et al., 2017) no trecho "o ganho significativo que é obtido pelo uso do resultado de Schaefer and Stoll [i.e. o método de 3-descenso]:" observa-se que a abordagem ingênua é sempre mais rápida que o 3-descenso.

\subsubsection{Elligator compartilhado e descompressão mais rápida}

Embora tenha sido mostrado que a abordagem ingênua é mais rápida que o 3-descenso na seção anterior, seu uso para geração de base de $E\left[3^{n}\right]$ incorre em um custo substancial que parece inevitável na compressão de chave. Curiosamente, o conhecimento obtido no processo (na forma dos contadores $r$ que especificam os pontos na construção do Elligator 2) poderia ser compartilhado entre Alice e Bob, acelerando o trabalho deste último na descompressão de chaves. Para um aumento muito modesto no tamanho da chave pública de Alice (por exemplo, um único byte extra para cada um dos dois pontos da base forneceria um espaço que só é excedido com probabilidade bem abaixo de $2^{-400}$ ), a geração da base $E\left[3^{n}\right]$ de Bob ficaria cerca de $32 \%$ mais rápida, e sua descompressão completa da chave de Alice se tornaria cerca de $24 \%$ mais rápida.

Conforme discutido ba Seção 4.4, a geração de base ingênua requere em média 9/8 tentativas de contrução para obter cada ponto na ordem certa individualmente, e o processo de construção do segundo ponto e verificação tem que ser repetido 4/3 vezes na média para garantir que o par de pontos constituam uma base. Isso significa que, se o custo de gerar cada candidato é $\mathbf{P}$, o custo esperado para se obter uma base é de aproximadamente $(21 / 8) \mathbf{P}+(4 / 3) \mathbf{C}+2 \mathbf{E}$.

No caso de Alice compartilhar os contadores do Elligator 2 que levam a uma base, o custo para Bob se tornaria $2 \mathbf{P}+\mathbf{C}+2 \mathbf{E}$ uma vez que ambos os cálculos do Elligator e do teste de independência linear se tornaria determinístico.

Isso representa um ganho de $1.47 \times$ vezes (ou $32 \%$ mais rápido) somente na geração de base e um ganho global de $1.4 \times$ vezes para a descompressão quando comparado com o método de 3-descenso sugerido em (COSTELLO et al., 2017).

Note que se Bob confia em Alice, i.e., a chave pública de Alice é autenticada, então $n-1$ triplicações podem ser evitadas uma vez que o teste de independência linear não é necessário (os contadores do Elligator devem formar uma base genuína). In this case the improvement is more drastic, the cost to generate a basis for $E\left[3^{n}\right]$ becomes $2 \mathbf{P}^{\prime}+2 \mathbf{E}$ where $\mathbf{P}^{\prime}=\mathbf{L}+m \mathbf{D}$ which represents a speedup of $2.86 \times$ over the previous 3 -descent approach. In this case, the task of decompression would get about $2 \times$ faster. Nesse caso o ganho é mais drástico: o custo para gerar uma base para $E\left[3^{n}\right]$ se torna $2 \mathbf{P}^{\prime}+2 \mathbf{E}$ onde $\mathbf{P}^{\prime}=\mathbf{L}+m \mathbf{D}$ o que representa em uma melhoria de $2.86 \times$ vezes sobre o método anterior de 3 -descenso e a tarefa de descompressão se torna aproximadamente $2 \times$ vezes mais rápida.

Observação. O mecanismo de encapsulamento de chave SIKE usa configurações ligeiramente 
diferentes em relação ao processamento da chave pública descompactada. No entanto, isso não afeta o processo de compressão e descompressão. Mais precisamente, a chave pública é sempre compactada na função de geração de chave e descompactada antes da função de encapsulamento de chave. Além disso, como o contador no Elligator compartilhado é calculado a partir de informações públicas, ele não afeta a segurança dos dois protocolos acima.

Além disso, os contadores Elligator 2 tendem a ser muito pequenos. Especificamente, se a probabilidade de que um candidato a ponto seja rejeitado em uma determinada tentativa for $1 / t$, então o número esperado de tentativas é $t /(t-1)$ e a probabilidade de que o número de tentativas exceda $N$ é a probabilidade de falhar $N$ vezes, ou seja, $1-(t-1) \sum k=1^{N} 1 / t^{k}=t^{-N}$. Para o primeiro ponto, $t=9$ (somente 1 dos 9 pontos de 3-torção causa rejeição ao calcular $\left[3^{n-1}\right] R$ ), enquanto para o segundo ponto, $t=3$ (pois a probabilidade de um candidato ser aceito em uma certa tentativa é $(8 / 9)(3 / 4)=2 / 3$, e portanto a probabilidade de rejeição é de $1 / 3)$. Portanto, para o primeiro ponto, o número esperado de tentativas é $9 / 8$ e a probabilidade de que o número de tentativas exceda $N$ é $9^{-N}$, enquanto para o segundo ponto o número esperado de tentativas é $3 / 2$ e a probabilidade de que o número de tentativas exceda $N$ é $3^{-N}$. Se for usado um byte para cada contador (interpretado como um índice para uma tabela de quadrados ou não-quadrados), as probabilidades de que o espaço disponível no contador $(N=255)$ seja excedido são $2^{-811.5} \mathrm{e}$

$2^{-404.2}$ respectivamente. Isso significa que Alice precisa transmitir apenas mais dois bytes com sua chave.

Esse aumento modesto no tamanho da chave de Alice é compensado por acelerações valiosas para Bob. Para $m=372$ e $n=239$, o tamanho da chave estendida de Alice torna-se 330 bytes em vez de 328 (COSTELLO et al., 2017, Tabela 3), correspondente ao tamanho original da chave de Bob. Enquanto o tempo para geração de uma bse de $3^{n}$-torção para Alice é o indicado na Tabela 6, o tempo de Bob é reduzido em cerca de 13.63 Mega ciclos resultando em uma razão de 1.47 em vez de 1.15. Além disso, a taxa de compressão de Alice em relação ao estado da arte anterior também permanece a mesma, mas a taxa de Bob melhora de 1.09 para 1.30.

\subsubsection{O Elligator compartilhado em bases emaranhadas}

Curiosamente, a técnica de geração de base binária emaranhada não só possibilita a descompressão com o Elligator compartilhado, mas também requer menos largura de banda extra em comparação com o caso ternário, ou seja, 1 em vez de 2 bytes extras. Além disso, a descompressão $50 \%$ mais rápida resultante é consideravelmente mais perceptível em comparação ao caso ternário. O motivo da melhoria é devido à possibilidade de se evitar um teste caro de quadraticidade no Etapa 1 do Algoritmo 2 de geração de base emaranhada. Nesse caso, Bob, que é responsável por compactar sua chave na torção $2^{m}$, pode transmitir a quadraticidade de $A$ por meio de um único bit $b$. Uma vez que o tamanho da chave pública de Bob não é um múltiplo exato de 32 bytes para um valor primo de 751-bit, alguns bits não utilizados na representação orientada a bytes da chave estão disponíveis e um deles pode ser usado para transmitir esta 
informação sobre $A$.

A outra informação que Bob pode compartilhar com Alice é o contador $r$ calculado na Etapa 4 que leva a um ponto na curva para o primeiro candidato. Isso pode ser feito com um único byte extra. A probabilidade de exceder um byte é a probabilidade da primeira abscissa falhar em estar na curva $N=255$ vezes, ou seja, $2^{-N}=2^{-255}$. Além disso, como o segundo ponto em uma base emaranhada é determinado pelo primeiro ponto, nenhum segundo byte extra é necessário neste caso. O Algoritmo 3 descreve a geração de base emaranhada com o Elligator compartilhado para descompressão.

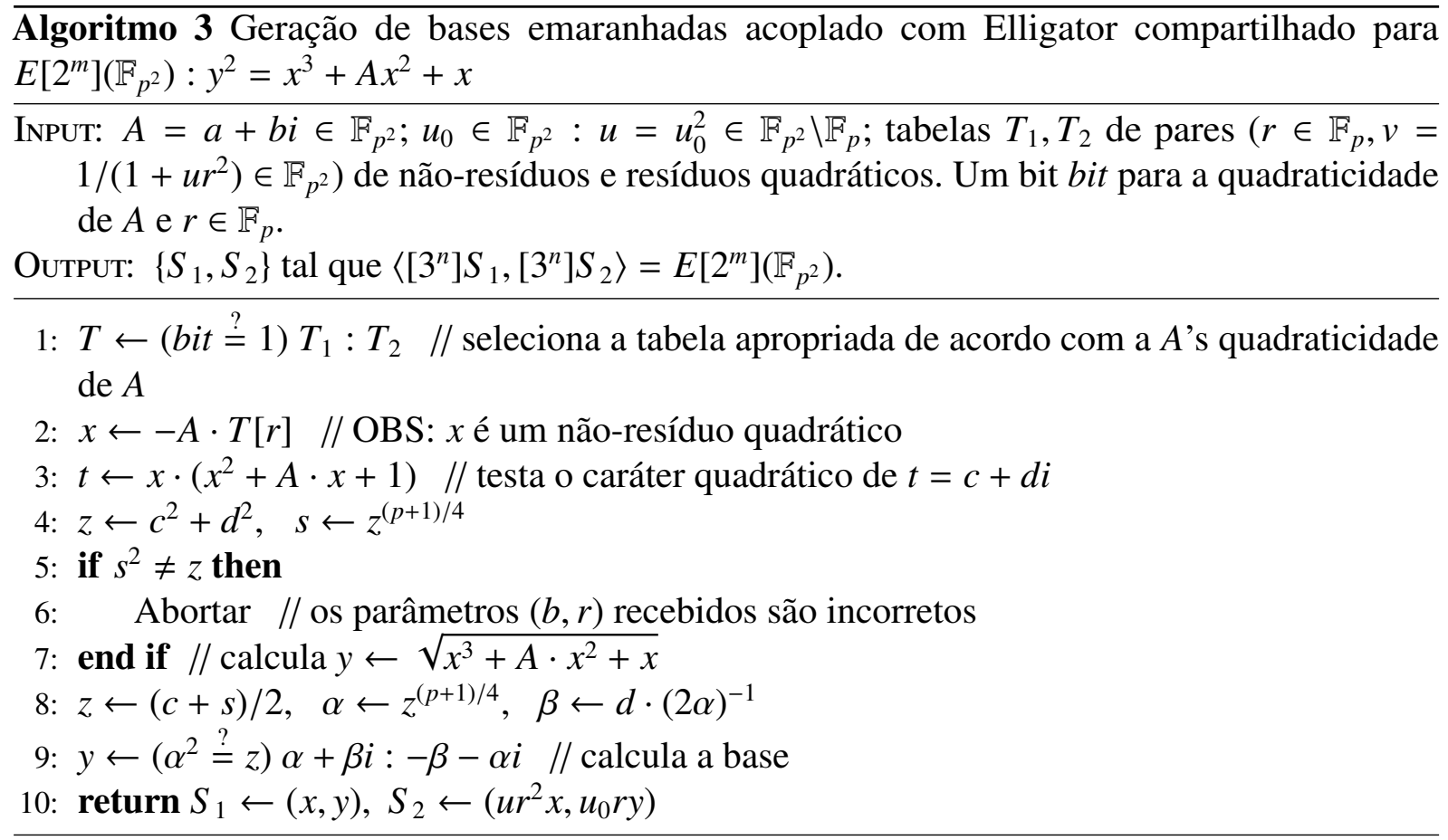

Base emaranhada + Elligator compartilhado: A contagem resultante de operações para a geração de base emaranhada acoplada com o Elligator compartilhado envolve uma única iteração do laço na Etapa 3 do Algoritmo 2 totalizando $(m+n+1) \mathbf{s}+(n+9) \mathbf{m}$ e a parte final (Etapas 8 a 10) totalizando $(m+n-1) \mathbf{s}+(n+6) \mathbf{m}+\mathbf{i}$. Somando os custos acima, a nova geração de bases tem custo

$$
2(m+n) \mathbf{s}+(2 n+15) \mathbf{m}+\mathbf{i} .
$$

Assumindo $\mathbf{i}=100 \mathbf{m}$ e $\mathbf{s}=0.8 \mathbf{m}$, isso representa um ganho de desempenho de $29.9 \times$ na geração de base quando comparado à geração de bases simples, descrita na Seção 2.

\subsection{Cálculo de emparelhamentos}

Calcular emparelhamentos é essencial para compressão de chaves. Emparelhamentos funcionam como um produto escalar, e projetam o logaritmo discreto elíptico num subgrupo 
multiplicativo de um corpo finito suave, com ordem da forma $p^{m}$ para um primo pequeno $p$ (no caso presente, $p \in\{2,3\}$ ). Isso possibilita o uso do algoritmo Pohlig-Hellman para calcular eficientemente esse logaritmo discreto, que possibilitará expressar as chaves de maneira compacta. Contudo, os emparelhamentos em questão estão entre as primitivas criptográficas computacionalmente mais pesadas, uma vez que as otimizações elaboradas nas últimas décadas para criptossistemas baseados em emparelhamentos foram projetadas para grupos elípticos onde o problema do logaritmo discreto é classicamente intratável, o que claramente não é (nem pode ser) a situação aqui. É fundamental, portanto, que o cálculo dos emparelhamentos seja o mais eficiente possível.

Apresentam-se agora novas melhorias no cálculo do emparelhamento de Tate (FREY; MüLLER; RüCK, 1999), que na prática é mais eficiente que o emparelhamento de Weil. Uma observação interessante da melhoria obtida é que, contrariamente à intuição baseada em pesquisas recentes (BERNSTEIN et al., 2012; CHUENGSATIANSUP et al., 2014; COSTELLO et al., 2017), o modelo tradicional de Weierstrass (SILVERMAN, 1986) revela-se mais eficiente para a aplicação presente que o modelo de Montgomery (MONTGOMERY, 1987).

Mais precisamente, as técnicas de cálculo de emparelhamento de Costello et al. (COSTELLO et al., 2017) baseiam-se em curvas numa variante do modelo de Montgomery, com quatro coordenadas projetivas $\left(X^{2}, X Z, Z^{2}, Y Z\right)$ em vez das duas coordenadas projetivas usuais daquele modelo. Esse escolha, segundo os autores, foi a melhor configuração encontrada dentre os vários modelos que eles investigaram. Na presente proposta, argumenta-se que o modelo mais antigo, e hoje em dia menos favorecido, de Weierstrass (especificamente, o modelo curto de Weierstrass) leva a algoritmos mais eficientes para o cálculo de emparelhamentos nas curvas supersingulares relevantes à criptografia baseada em isogenias.

É interessante notar que Costello et al. descartam de antemão a técnica de eliminação de denominadores (BARRETO et al., 2002), e mantêm numeradores e denominadores separados durante o cálculo do emparelhamento. Contudo, os valores do emparelhamento estão definidos em $\mathbb{F}_{p^{2}}$ e o inverso de um elemento $a+b i$ desse corpo é $(a-b i) /\left(a^{2}+b^{2}\right)$. Logo, em vez de manter separado o denominador de $z /(a+b i)$ (para algum numerador $z$ ), pode-se simples e imediatamente multiplicar o valor do emparelhamento pelo conjugado $a-b i$; o resultado difere do original apenas por um denominador que consiste na norma $a^{2}+b^{2} \in \mathbb{F}_{p}$, e esse denominador é efetivamente eliminado pela exponenciação final do emparelhamento reduzido de Tate. Isso deixa o custo do cálculo do emparelhamento intacto, mas simplifica a implementação por remover inteiramente a necessidade de manter numeradores e denominadores separados.

Seja $r \geq 0$ a ordem do emparelhamento. Para o grau de mergulho $k=1, r \mid \Phi_{1}\left(p^{2}\right)=$ $p^{2}-1=2^{m} \cdot 3^{n} \cdot(p-1)$, e por construção $r$ é sempre $2^{m}$ ou $3^{n}$. O foco aqui será calcular o emparelhamento reduzido de Tate de ordem $r$, cujo primeiro argumento precisa ter essa mesma ordem. Para o propósito de comprimir chaves SIDH, quatro emparelhamentos das seguintes formas são calculados juntos (um quinto emparelhamento $e_{0}:=\mathrm{e}_{r}(P, Q)=\mathrm{e}_{r}\left(P_{0}, Q_{0}\right)^{\operatorname{deg} \phi}$ é feito 
disponível por cálculo prévio ao tempo de execução):

$$
\begin{aligned}
& e_{1}:=\mathrm{e}_{r}\left(P, R_{1}\right), e_{2}:=\mathrm{e}_{r}\left(P, R_{2}\right), \\
& e_{3}:=\mathrm{e}_{r}\left(Q, R_{1}\right), e_{4}:=\mathrm{e}_{r}\left(Q, R_{2}\right)
\end{aligned}
$$

onde os primeiros dois emparelhamentos compartilham o mesmo primeiro argumento $P$, e os dois emparelhamentos seguintes compartilham o mesmo primeiro argumento $Q$.

De agora em diante, a discussão será dividida em dois casos: emparelhamentos de ordem binária, $r=2^{m}$, e emparelhamentos de ordem ternária, $r=3^{n}$. A equação da curva no modelo curto de Weierstrass é $E_{W}: v^{2}=u^{3}+a u+b$. Dada uma curva no modelo de Montgomery $E_{M}: y^{2}=x^{3}+A x^{2}+x$, o modelo curto de Weierstrass correspondente é obtido via $a=1-A^{2} / 3$, $b=\left(2 A^{3}-9 A\right) / 27$, e um ponto $(x, y) \in E_{M}$ é mapeado a um ponto $(u, v) \in E_{W}$ fazendo $u=x+A / 3, v=y$. Por conveniência, estende-se aqui as coordenadas jacobianas comuns $[X: Y: Z]$ com um quarto componente, $[X: Y: Z: T]$ onde $T=Z^{2}$.

\subsubsection{Emparelhamentos de ordem binária}

O cálculo do emparelhamento reduzido de Tate $\mathrm{e}_{r}(P, Q)$ de ordem $r=2^{m}$ procede conforme descrito no Algoritmo 4, que requer dobrar um ponto $V \in E\left(\mathbb{F}_{p^{2}}\right)$. As fórmulas de duplicação em coordenadas jacobianas têm uma única exceção, que ocorre quando o ponto sendo dobrado tem ordem 2. Isto só acontece quando $y=0$, uma vez que o coeficiente angular da tangente à curva nesse ponto torna-se indefinida. Essa exceção, porém, só pode ocorrer deterministicamente no cenário aqui contemplado, mais precisamente no último passo do laço de Miller, uma vez que, por definição, o primeiro argumento do emparelhamento é sempre um ponto de ordem $2^{m}$, escolhido pela própria entidade que está calculando o emparelhamento (portanto, sem interferência externa).

Além disso, a diferença no tempo de execução desse passo e dos demais não revela nenhuma informação privada, já que os argumentos do emparelhamento já são públicos (em virtude de pertencerem a uma base convencional de torção) ou serão tornados públicos como parte da própria chave pública.

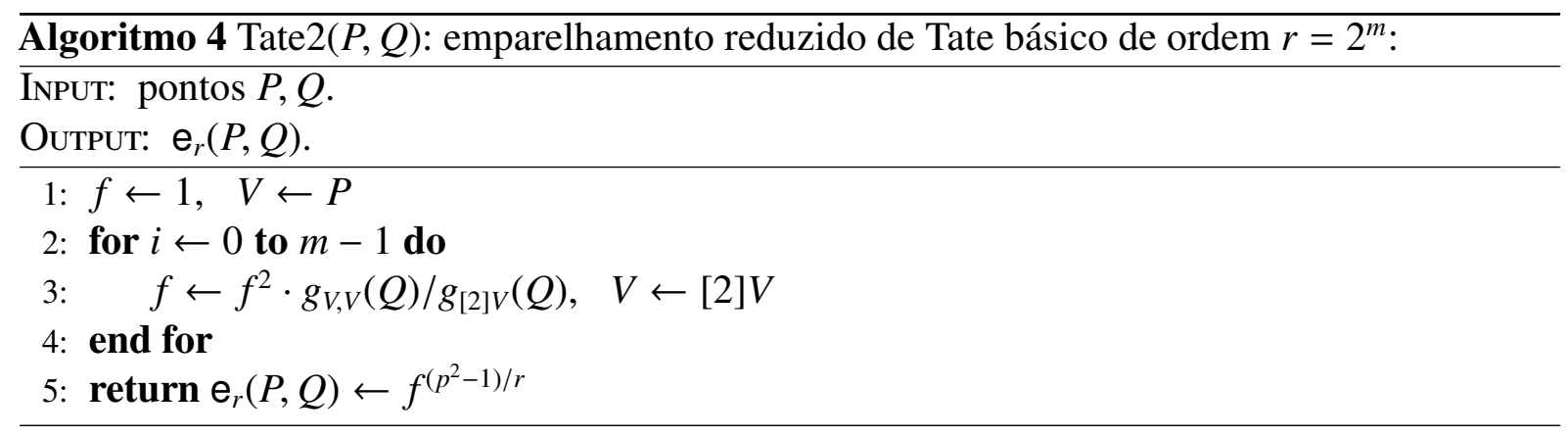

No Algoritmo 4, a função $g_{U, V}$ é definida como uma reta através de $U$ e $V$. Se $U=V$ então $g_{U, V}$ é a tangente em $U$, e se $U=\infty$ ou $V=\infty$ então $g_{U, V}$ é uma reta vertical no outro ponto. Também denota-se por $g_{U}$ o valor $g_{U,-U}$. 
O algoritmo mais eficiente de duplicação de pontos para coordenadas Jacobianas (simples ou modificadas) parece ser o inventado por Bernstein e Lange (BERNSTEIN; LANGE, 2008), que mantém uma coordenada adicional $U:=a Z^{4}$. No entanto, esse algoritmo não calcula diretamente o valor de $T=Z^{2}$ que será necessário para cálculo de emparelhamento. Assim, as fórmulas de Bernstein-Lange foram modificadas de modo que o custo de recuperar $T$ seja menor do que elevar $Z$ ao quadrado.

Seja $V=[X: Y: Z: U: T]$ e $[2] V=\left[X^{\prime}: Y^{\prime}: Z^{\prime}: U^{\prime}: T^{\prime}\right]$ no sistema de coordenadas estendidas definido acima. Então, inicializando $T \leftarrow Z^{2}$ e $U \leftarrow a T^{2}$ :

$$
\begin{aligned}
& X_{2} \leftarrow X^{2} ; Y_{2} \leftarrow Y^{2} ; W \leftarrow 2 Y_{2} ; W_{2} \leftarrow W^{2} ; \\
& M \leftarrow 3 X_{2}+U ; S \leftarrow(X+W)^{2}-X_{2}-W_{2} ; \\
& X^{\prime} \leftarrow M^{2}-2 S ; Y^{\prime} \leftarrow M \cdot\left(S-X^{\prime}\right)-2 W_{2} ; \\
& Z^{\prime} \leftarrow(Y+Z)^{2}-Y_{2}-T ; T^{\prime} \leftarrow\left(Z^{\prime}\right)^{2} ; U^{\prime} \leftarrow 4 W_{2} \cdot U ;
\end{aligned}
$$

$O$ custo é $2 \mathbf{M}+7 \mathbf{S}+16 \mathbf{A}=20 \mathbf{m}+63 \mathbf{a}$, o que é apenas $1 \mathbf{m}+5 \mathbf{a}$ a mais do que duplicar sem ter que produzir $Z^{2}$ como subproduto: $3 \mathbf{M}+5 \mathbf{S}+14 \mathbf{A}=19 \mathbf{m}+58 \mathbf{a}$.

$\mathrm{O}$ algoritmo produz os valores intermediários $\lambda_{N}:=M, \lambda_{D}:=Z^{\prime}=2 Y Z$, $W$, e $Y_{2}$, além das coordenadas do ponto. Esse valores, juntos com $L \leftarrow Z^{\prime} \cdot T, R \leftarrow Z^{\prime} \cdot \bar{T}$, são úteis no cálculo da função equivalente a $g_{V, V}(Q) / g_{[2] V}(Q)$, a saber, $\tilde{g}_{2}(V, Q):=(M \cdot(T \cdot x-X)+W-L \cdot y) \cdot R$. $\left(T^{\prime} \cdot x-X^{\prime}\right)^{-}$quando $V \neq O$ e [2] $V \neq O$ (i.e. $Z \neq 0$ e $\left.Z^{\prime} \neq 0\right), \tilde{g}_{2}(V, Q):=(T \cdot x-X) \cdot \bar{T}$ quando $V=-V \neq O$ (i.e. $Z \neq 0$ e $Z^{\prime}=0$ ), ou simplesmente $\tilde{g}_{2}(V, Q):=1$ quando $Z=O$.

Denominadores no corpo base, dado por $\left|Z^{2} \cdot\left(T^{\prime} \cdot x-X^{\prime}\right)\right|^{2} \in \mathbb{F}_{p}$ no primeiro caso e por $\left|Z^{2}\right|^{2} \in \mathbb{F}_{p}$ no segundo caso, são eliminados.

Como o cenário onde ocorre os cálculos do emparelhamento envolve somente bases do grupo de $2^{m}$-torção, e portanto pontos de ordem completa $2^{m}$, a fórmula excepcional nunca é utilizada de fato até o fim do laço de Miller. A diferença no tempo de processamento é irrelevante à segurança aqui, já que as informações envolvidas nos cálculos são públicas.

Entretanto, é possível otimizar os cálculos de (uma função equivalente a) $\tilde{g}_{2}(V, Q)$. Primeiro, a expressão $\left(T^{\prime} \cdot x-X^{\prime}\right)$ que ocorre em um certo passo fará o papel de $(T \cdot x-X)$ no próximo passo, então pode-se simplesmente armazená-lo entre um passo e outro, e assim economizar 1M. Segundo, pode-se mostrar que todos fatores $R$ e $\bar{T}$ que aparecem na definição de $\tilde{g}_{2}(V, Q)$ são irrelevantes ao valor do emparelhamento, e podem ser omitidos.

Consequentemente, inicializando-se $h \leftarrow T \cdot x-X$ antes do laço de Miller ao custo de $1 \mathbf{M}$ por emparelhamento, a função de linha pode ser avaliada como:

$$
g \leftarrow M \cdot h+W-L \cdot y ; h \leftarrow T^{\prime} \cdot x-X^{\prime} ; g \leftarrow g \cdot \bar{h}
$$

pelo custo de $4 \mathbf{M}+3 \mathbf{A}=12 \mathbf{m}+26 \mathbf{a}$ por passo do laço de Miller. $O$ custo de calcular somente $L \leftarrow Z^{\prime} \cdot T$ é de $1 \mathbf{M}=3 \mathbf{m}+5 \mathbf{a}$. Isso conclui a construção de uma função de linha $\hat{g}_{2}(V, Q)$ equivalente a $\tilde{g}_{2}(V, Q)$. 
A atualização de $f$ a cada passo como $f \leftarrow f^{2} \cdot \hat{g}_{2}(V, Q)$ incorre em $2 \mathbf{m}+3 \mathbf{a}$ para calcular o quadrado complexo $f^{2}$ mais $3 \mathbf{m}+5 \mathbf{a}$ para calcular $f^{2} \cdot \hat{g}_{2}(V, Q)$ a partir de $f^{2}$ e $\hat{g}_{2}(V, Q)$, totalizando $5 \mathbf{m}+8 \mathbf{a}$. Portanto, a variante proposta tem o seguinte custo total por passo:

- custo (compartilhado) da duplicação de ponto e construção da função de linha: $20 \mathbf{m}+$ $63 \mathbf{a}+3 \mathbf{m}+5 \mathbf{a}=23 \mathbf{m}+68 \mathbf{a}$

- custo (individual) da avaliação e acúmulo da função de linha: $12 \mathbf{m}+26 \mathbf{a}+5 \mathbf{m}+8 \mathbf{a}=$ $17 \mathbf{m}+34 \mathbf{a}$.

Por comparação, a técnica utilizada por Costello et al. (COSTELLO et al., 2017) possui os seguintes custos:

- custo (compartilhado) da duplicação de ponto e construção da função de linha: $9 \mathbf{M}+5 \mathbf{S}+$ $1 \mathbf{s}+7 \mathbf{a}=37 \mathbf{m}+1 \mathbf{s}+67 \mathbf{a}$

- custo (individual) da avaliação e acúmulo da função de linha: $5 \mathbf{M}+2 \mathbf{S}+2 \mathbf{s}+1 \mathbf{a}=$ $19 \mathbf{m}+2 \mathbf{s}+32 \mathbf{a}$

Logo, no presente caso, onde se tem que computar pares de emparelhamentos que compartilham o mesmo primeiro argumento, a técnica proposta custa uma fração $\approx(23+2$. $17) /(37.8+2 \cdot 20.6)=57 / 79 \approx 72 \%$ do método de Costello et al., assumindo $1 \mathrm{~s} \approx 0.8 \mathbf{m} \mathrm{e}$ essencialmente ignorando a.

\subsubsection{Emparelhamentos numa base emaranhada}

Se dois emparelhamentos $e\left(P, R_{1}\right), e\left(P, R_{2}\right)$ que compartilham o mesmo primeiro argumento $P$ são calculados numa base emaranhada $R_{1}=\left(x_{1}, y_{1}\right), R_{2}=\left(x_{2}, y_{2}\right) \operatorname{com} x_{2}=t^{2} \cdot x_{1}$, $y_{2}=t \cdot y_{1}$, pode-se melhorar ligeiramente o cálculo e o acúmulo da função de linha, explorando para isso o fato que a multiplicação por valores $t$ ou $t^{2}$ escolhidos cuidadosamente a partir dos valores $T^{\prime} \cdot x_{1}$ ou $L \cdot y_{1}$ é mais barato que as multiplicações completas $T^{\prime} \cdot x_{2}$ ou $L \cdot y_{2}$ para um ponto $\left(x_{2}, y_{2}\right)$ genérico.

Especificamente, para $t=(1+i) r$ e $t^{2}=2 i r^{2}$ com algum valor $r \in \mathbb{F}_{p}$ pequeno, o custo de uma implementação dedicada de emparelhamentos simultâneos em bases emaranhadas reduz-se por $2 \mathbf{m}+10 \mathbf{a}$, tornando-se portanto apenas $55 \mathbf{m}+126 \mathbf{a}$, ou menos de $70 \%$ do custo do método de Costello et al.

A Tabela 3 traz um sumário das melhorias de desempenho proporcionadas pelas técnicas propostas acima. 


\begin{tabular}{cccc}
\hline emparelhamentos & Costello et al. & este trabalho & razão \\
\hline 1 & $56 \mathbf{m}+3 \mathbf{s}+99 \mathbf{a}$ & $40 \mathbf{m}+102 \mathbf{a}$ & $\approx 0.685$ \\
2 & $75 \mathbf{m}+5 \mathbf{s}+131 \mathbf{a}$ & $57 \mathbf{m}+136 \mathbf{a}$ & $\approx 0.722$ \\
$2^{\dagger}$ & $75 \mathbf{m}+5 \mathbf{s}+131 \mathbf{a}$ & $55 \mathbf{m}+126 \mathbf{a}$ & $\approx 0.696$ \\
\hline
\end{tabular}

† emparelhamentos simultâneos em bases emaranhadas

Tabela 3 - Custo do laço de Miller binário (a razão assume $\mathbf{s} \approx 0.8 \mathbf{m}$ e ignora $\mathbf{a}$ ).

A variante proposta para o cálculo de emparelhamentos reduzidos simultâneos de Tate é descrita em detalhes no Algoritmo 5.

\subsubsection{Emparelhamentos de ordem ternária}

O cálculo do emparelhamento reduzido de Tate $\mathrm{e}_{r}(P, Q)$ de ordem $r=3^{n}$ ocorre conforme descrito no Algoritmo 6. Novamente, as fórmulas de triplicação em coordenas Jacobianas possuem uma exceção quando $y=0$, mas pode-se lidar com isso de forma similar ao caso binário. A diferença no tempo de execução não revela nenhuma informação privada pela mesma razão: apenas dados públicos são utilizados no cálculo dos emparelhamentos.

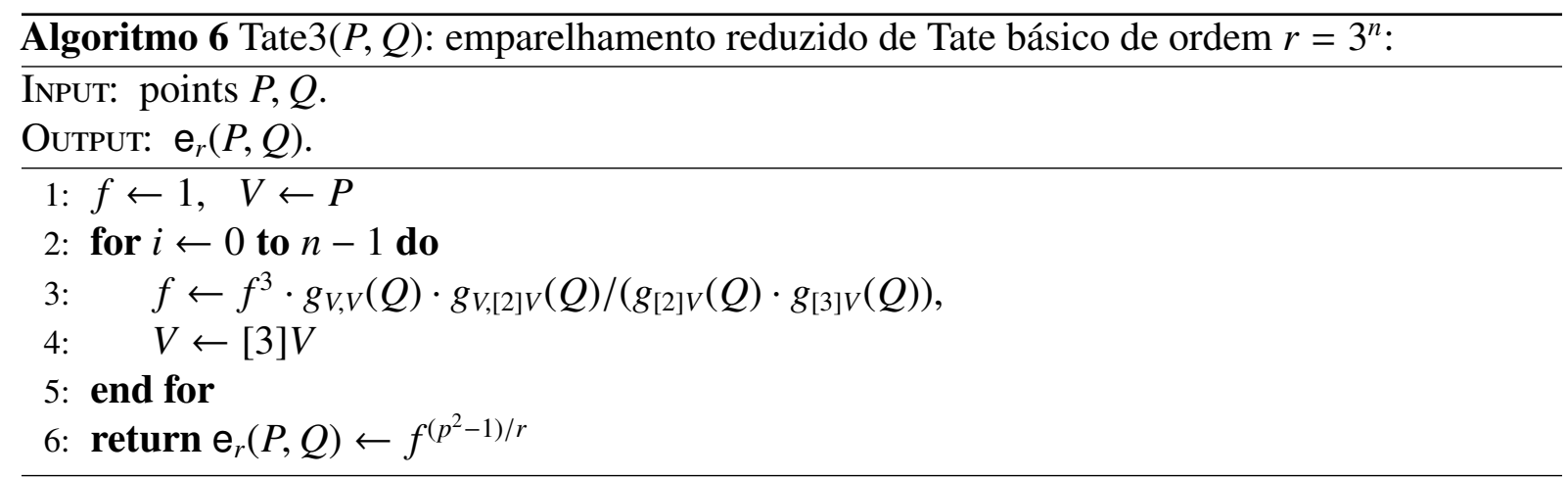

O algoritmo mais eficiente de triplicação de pontos conhecido parece ser o inventado por Bernstein e Lange (BERNSTEIN; LANGE, 2008). Seu custo é $6 \mathbf{M}+10 \mathbf{S}+25 \mathbf{A}=38 \mathbf{m}+110 \mathbf{a}$ para uma curva com coeficientes genéricos na equação. Apresenta-se uma variante para as mesmas coordenadas Jacobianas utilizadas no caso binário, com custo de $5 \mathbf{M}+11 \mathbf{S}+31 \mathbf{A}=37 \mathbf{m}+120 \mathbf{a}$.

Seja $V=[X: Y: Z: T: U]$ e $[3] V=\left[X^{\prime}: Y^{\prime}: Z^{\prime}: T^{\prime}: U^{\prime}\right]$ no mesmo sistema de 
$\overline{\text { Algoritmo } 5 \text { Tate } 2\left(P,\left[Q_{j}\right], m\right) \text { : emparelhamento reduzido de Tate de ordem } r=2^{m}}$

INPUT: Curva $E: y^{2}=x^{3}+a x+b$

- Ponto $P=\left[X_{P}: Y_{P}: Z_{P}\right]$ em $E$ de ordem $2^{m}$

- $t$ pontos $Q_{j}=\left[X_{Q_{j}}: Y_{Q_{j}}: Z_{Q_{j}}\right]$ em $E, Z_{Q_{j}} \in\{0,1\}$

OutPut: Lista de $t$ valores $e_{2^{m}}\left(P, Q_{j}\right)$

1: $X \leftarrow X_{P} ; \quad Y \leftarrow Y_{P} ; \quad Z \leftarrow Z_{P} ; \quad T \leftarrow Z^{2}$

$\triangleright$ NB: as seguintes operações ocorrem em $\mathbb{F}_{p^{2}}$

2: for $j \leftarrow 0$ to $t-1$ do

3: $\quad f_{j} \leftarrow 1 ; \quad h_{j} \leftarrow T \cdot X_{Q_{j}}-X$;

4: end for

5: for $k \leftarrow 0$ to $m-1$ do

$\triangleright$ duplicação de pontos e construção da função de linha:

6: $\quad X_{2} \leftarrow X^{2} ; \quad Y_{2} \leftarrow Y^{2} ; \quad Y_{4} \leftarrow Y_{2}^{2}$

7: $\quad M \leftarrow 3 X_{2}+a \cdot T^{2}$

8: $\quad S \leftarrow 2\left(\left(X+Y_{2}\right)^{2}-X_{2}-Y_{4}\right)$

9: $\quad X^{\prime} \leftarrow M^{2}-2 S$

10: $\quad Y^{\prime} \leftarrow M \cdot\left(S-X^{\prime}\right)-8 Y_{4}$

11: $\quad Z^{\prime} \leftarrow(Y+Z)^{2}-Y_{2}-T$;

12: $\quad T^{\prime} \leftarrow\left(Z^{\prime}\right)^{2} ; \quad L \leftarrow Z^{\prime} \cdot T ; \quad W \leftarrow 2 Y_{2}$

13: $\quad$ if $Z^{\prime}=0$ then // exceção para pontos em [2]E

14: $\quad X^{\prime} \leftarrow 0 ; \quad Y^{\prime} \leftarrow 1$

15: $\quad$ end if

$\triangleright$ avaliação e acúmulo da função de linha:

16: $\quad$ for $j \leftarrow 0$ to $t-1$ do

17: $\quad$ if $Z^{\prime} \neq 0$ then

18: $\quad g \leftarrow M \cdot h_{j}+W-L \cdot Y_{Q_{j}}$

19: $\quad h_{j} \leftarrow T^{\prime} \cdot X_{Q_{j}}-X^{\prime}$

20: $\quad g \leftarrow g \cdot h_{j}^{*}$

21: $\quad$ else

22: $\quad g \leftarrow h_{j}$;

23: $\quad$ end if

24: $\quad f_{j} \leftarrow f_{j}^{2} ; \quad f_{j} \leftarrow f_{j} \cdot g ;$

25: $\quad$ end for

26: $\quad X \leftarrow X^{\prime} ; \quad Y \leftarrow Y^{\prime}$;

27: $\quad Z \leftarrow Z^{\prime} ; \quad T \leftarrow T^{\prime}$;

28: end for

29: return $\left[\left(Z_{Q_{j}} \stackrel{?}{\neq} 0\right) f_{j}^{\left(p^{2}-1\right) / r}: 1 \mid j=0 \ldots t-1\right]$ 
coordenadas estendidas anterior. Então, inicializando $T \leftarrow Z^{2}$ e $U \leftarrow a T^{2}$ :

$$
\begin{aligned}
& X_{2} \leftarrow X^{2} ; Y_{2} \leftarrow Y^{2} ; Y_{4} \leftarrow Y_{2}^{2} ; \\
& M \leftarrow 3 X_{2}+U ; M_{2} \leftarrow M^{2} ; \\
& D \leftarrow\left(X+Y_{2}\right)^{2}-X_{2}-Y_{4} ; F \leftarrow 6 D-M_{2} ; \\
& F_{2} \leftarrow F^{2} ; W \leftarrow 2 Y_{2} ; W^{\prime} \leftarrow 2 W ; S \leftarrow 16 Y_{4} ; \\
& G \leftarrow(M+F)^{2}-M_{2}-F_{2}-S ; G^{\prime} \leftarrow S-G ; \\
& H \leftarrow 2 F_{2} ; H_{2} \leftarrow H^{2} ; H^{\prime} \leftarrow 4 G ; F^{\prime} \leftarrow 2 F ; \\
& X^{\prime} \leftarrow(X+H)^{2}-X_{2}-H_{2}-W^{\prime} \cdot H^{\prime} ; \\
& Y^{\prime} \leftarrow 2 Y \cdot\left(H^{\prime} \cdot G^{\prime}-F^{\prime} \cdot H\right) ; Z^{\prime} \leftarrow(Z+F)^{2}-T-F_{2} ; \\
& T^{\prime} \leftarrow\left(Z^{\prime}\right)^{2} ; \quad U^{\prime} \leftarrow 4 H_{2} \cdot U
\end{aligned}
$$

Esse algoritmo gera valores intermediários $F, F^{\prime}, G^{\prime}, W, W^{\prime}$, e $M$, além das coordenadas do ponto. Esses valores, juntos com $L \leftarrow\left((Y+Z)^{2}-Y_{2}-T\right) \cdot T$ e $R \leftarrow F \cdot \bar{T}$, são úteis para no cálculo da função equivalente a $g_{V, V}(Q) \cdot g_{V,[2] V}(Q) /\left(g_{[2] V}(Q) \cdot g_{[3] V}(Q)\right)$, onde $\tilde{g}_{3}(V, Q):=$ $(M \cdot h+d) \cdot\left(G^{\prime} \cdot h+F^{\prime} \cdot d\right) \cdot\left(W^{\prime} \cdot h+F\right)^{-} \cdot R \cdot \overline{h_{3}}$ quando [3]V $\neq O ; \tilde{g}_{3}(V, Q):=(M \cdot h+d) \cdot \bar{L}$ quando [3] $V=O$ mas $V \neq O$; ou simplesmente $\tilde{g}_{3}(V, Q):=1$ quando $V=O$, onde $h:=T \cdot x-X$, $d:=W-L \cdot y, h_{3}:=T^{\prime} \cdot x-X^{\prime}$.

Pode-se otimizar ainda mais o cálculo de uma função $\hat{g}_{3}(V, Q)$ equivalente a $\tilde{g}_{3}(V, Q)$ de uma forma semelhante ao que foi feito para o caso binário. Primeiro, a expressão $T^{\prime} \cdot x-X^{\prime}$ que ocorre em uma determinada etapa desempenhará o papel de $T \cdot x-X$ na próxima etapa, portanto, pode-se simplesmente armazená-la de uma etapa para a próxima e, portanto, economizar $1 \mathbf{M}$. Em segundo lugar, pode-se mostrar que todos os fatores $R$ e $\bar{L}$ que aparecem na definição de $\tilde{g}_{3}(V, Q)$ são irrelevantes para o valor do emparelhamento, e podem ser omitidos.

Consequentemente, a construção da função parábola pode ser concluída calculando apenas $L$ conforme indicado acima, a um custo de $1 \mathbf{M}+1 \mathbf{S}+3 \mathbf{A}$. Após inicializar $h \leftarrow T \cdot x-X$ antes do laço de Miller a um custo de $1 \mathbf{M}$ por emparelhamento, o valor $g$ da função parábola pode ser avaliado como

$$
\begin{aligned}
& d \leftarrow W-L \cdot y ; \\
& g \leftarrow(M \cdot h+d) \cdot\left(G^{\prime} \cdot h+F^{\prime} \cdot d\right) \cdot\left(W^{\prime} \cdot h+F\right) ; \\
& h \leftarrow T^{\prime} \cdot x-X^{\prime} ; \\
& g \leftarrow g \cdot \bar{h} ;
\end{aligned}
$$

a um custo de $9 \mathbf{M}+5 \mathbf{A}$ por etapa do laço de Miller, exceto na etapa final, quando é simplesmente $g \leftarrow M \cdot h+d$. Isso completa a construção de uma função de parábola $\hat{g}_{3}(V, Q)$ equivalente a $\tilde{g}_{3}(V, Q)$.

A atualização de $f$ em cada etapa como $f \leftarrow f^{3} \cdot \hat{g}_{3}(V, Q)$ incorre em um custo 1 C para calcular o cubo complexo $f^{3}$, mais $1 \mathbf{M}$ para calcular $f^{3} \cdot \hat{g}_{3}(V, Q)$ de $f^{3}$ e $\hat{g}_{3}(V, Q)$. Portanto, a 


\begin{tabular}{cccc}
\hline emparelhamentos & Costello et al. & este trabalho & razão \\
\hline 1 & $103 \mathbf{m}+6 \mathbf{s}+188 \mathbf{a}$ & $72 \mathbf{m}+2 \mathbf{s}+200 \mathbf{a}$ & $\approx 0.683$ \\
2 & $137 \mathbf{m}+6 \mathbf{s}+248 \mathbf{a}$ & $104 \mathbf{m}+2 \mathbf{s}+266 \mathbf{a}$ & $\approx 0.756$ \\
\hline
\end{tabular}

Tabela 4 - Custo do laço ternário de Miller (a razão assume $\mathbf{s} \approx 0.8 \mathbf{m}$ e ignora $\mathbf{a}$ ).

variante proposta tem o seguinte custo global por etapa, onde novamente a parte compartilhada é amortizada entre emparelhamentos simultâneos que compartilham o mesmo primeiro argumento:

- custo (compartilhado) da triplicação de ponto e construção da função parábola: $5 \mathbf{M}+11 \mathbf{S}+$ $31 \mathbf{A}+1 \mathbf{M}+1 \mathbf{S}+3 \mathbf{A}=40 \mathbf{m}+134 \mathbf{a}$

- custo (individual) custo de avaliação e acúmulo da função parábola: $9 \mathbf{M}+5 \mathbf{A}+1 \mathbf{C}=$ $32 \mathbf{m}+2 \mathbf{s}+66 \mathbf{a}$.

Por comparação, a técnica (COSTELLO et al., 2017) possui os seguintes custos:

- custo (compartilhado) da triplicação de ponto e construção da função parábola: $19 \mathbf{M}+$ $6 \mathbf{S}+6 \mathbf{s}+15 \mathbf{a}=69 \mathbf{m}+6 \mathbf{s}+128 \mathbf{a}$

- custo (individual) custo de avaliação e acúmulo da função parábola: $10 \mathbf{M}+2 \mathbf{S}+4 \mathbf{a}=$ $34 \mathbf{m}+60 \mathbf{a}$.

Portanto, no presente caso, onde se deve calcular pares de emparelhamentos que compartilham o mesmo primeiro argumento, a técnica obtida custa uma fração $\approx(40+2 \cdot 33.6) /(73.8+2$. $34)=107.2 / 141.8 \approx 76 \%$ do método de Costello et al., assumindo $1 \mathbf{s} \approx 0.8 \mathbf{m}$ e essencialmente ignorando a.

As melhorias de desempenho trazidas pelas técnicas propostas estão resumidas na Tabela 4.

A variante proposta dos emparelhamento reduzido de Tate simultâneos é mostrado mais detalhadamente no Algoritmo 7.

\subsection{Cálculo de logaritmos discretos}

Seja $L:=\ell^{w}$ para algum inteiro $w>0$, e seja $\mu_{L^{e}} \subset \mathbb{F}_{p^{2}}$ o conjunto de $L^{e}$-ésimas raízes da unidade em $\mathbb{F}_{p^{2}}$, i.e. $\mu_{L^{e}}:=\left\{v \in \mathbb{F}_{p^{2}} \mid v^{L^{e}}=1\right\}$. Uma inversão em $\mu_{L^{e}}$ é simplesmente obter o conjugado, $(a+b i)^{-1}=a-b i$ uma vez que a norma é 1 . O método Pohlig-Hellman (Algoritmo 8) (POHLIG; HELLMAN, 1978), que calcula o logaritmo discreto de $c \in \mu_{L^{e}}$, requer a resolução de uma equação da forma

$$
r_{k}^{L^{e-1-k}}=s^{d_{k}}
$$


Algoritmo 7 Tate $3\left(P,\left[Q_{j}\right], n\right)$ : emparelhamento reduzido de Tate de ordem $r=3^{n}$

InPUT: Curva $E: y^{2}=x^{3}+a x+b$

- Ponto $P=\left[X_{P}: Y_{P}: Z_{P}\right]$ em $E$ de ordem $3^{n}$

- $t$ pontos $Q_{j}=\left[X_{Q_{j}}: Y_{Q_{j}}: Z_{Q_{j}}\right]$ em $E, Z_{Q_{j}} \in\{0,1\}$

Output: Lista de $t$ valores $e_{3^{n}}\left(P, Q_{j}\right)$

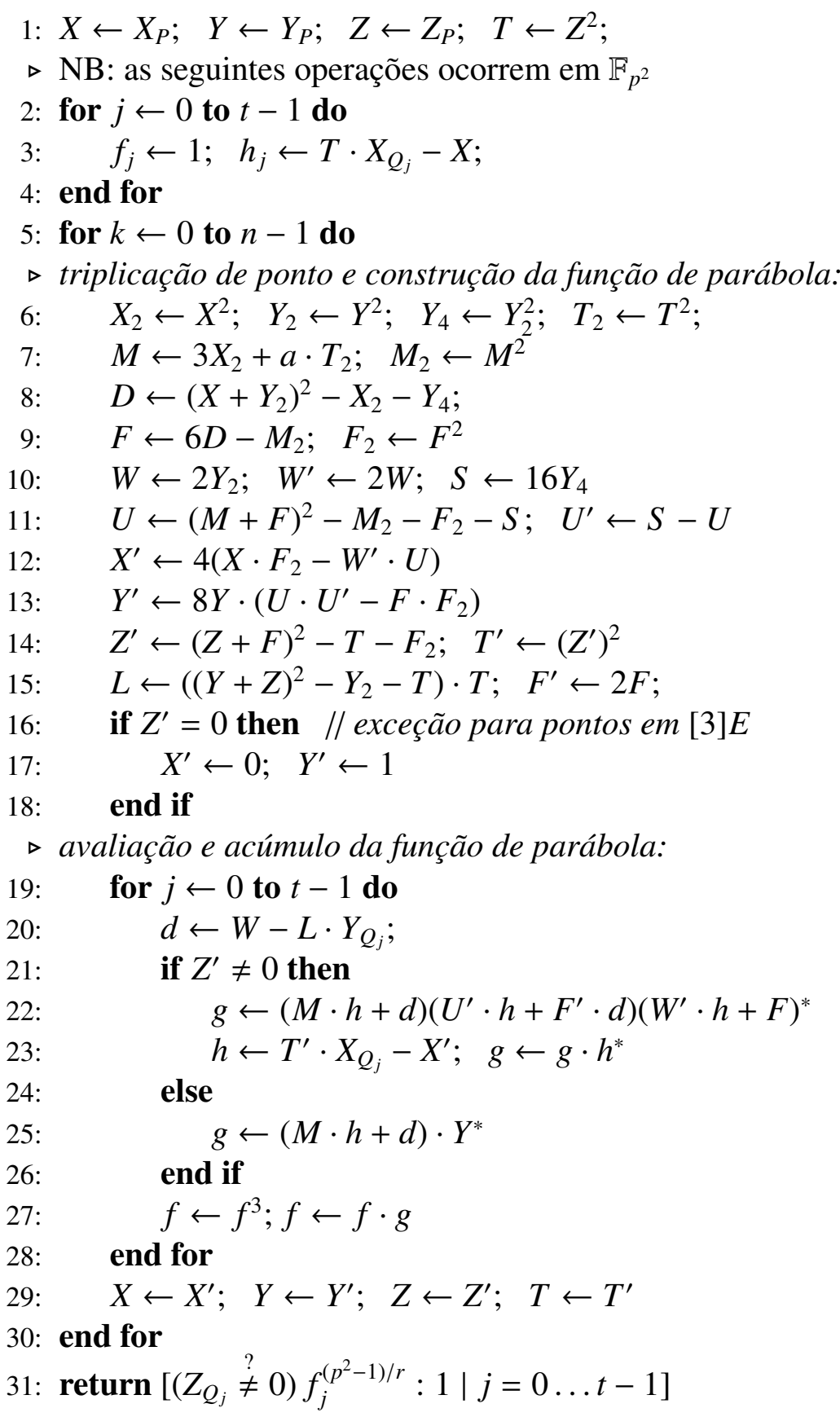


onde $g$ é um gerador fixo do grupo, $s=g^{L^{e-1}}$ possui ordem $L$ e, para $k=0, \ldots, e-1, d_{k} \in$ $\{0, \ldots, L-1\}$ é um dígito $L$-ário, $r_{0}=c$, e $r_{k+1}$ depende de $r_{k}$ e $d_{k}$.

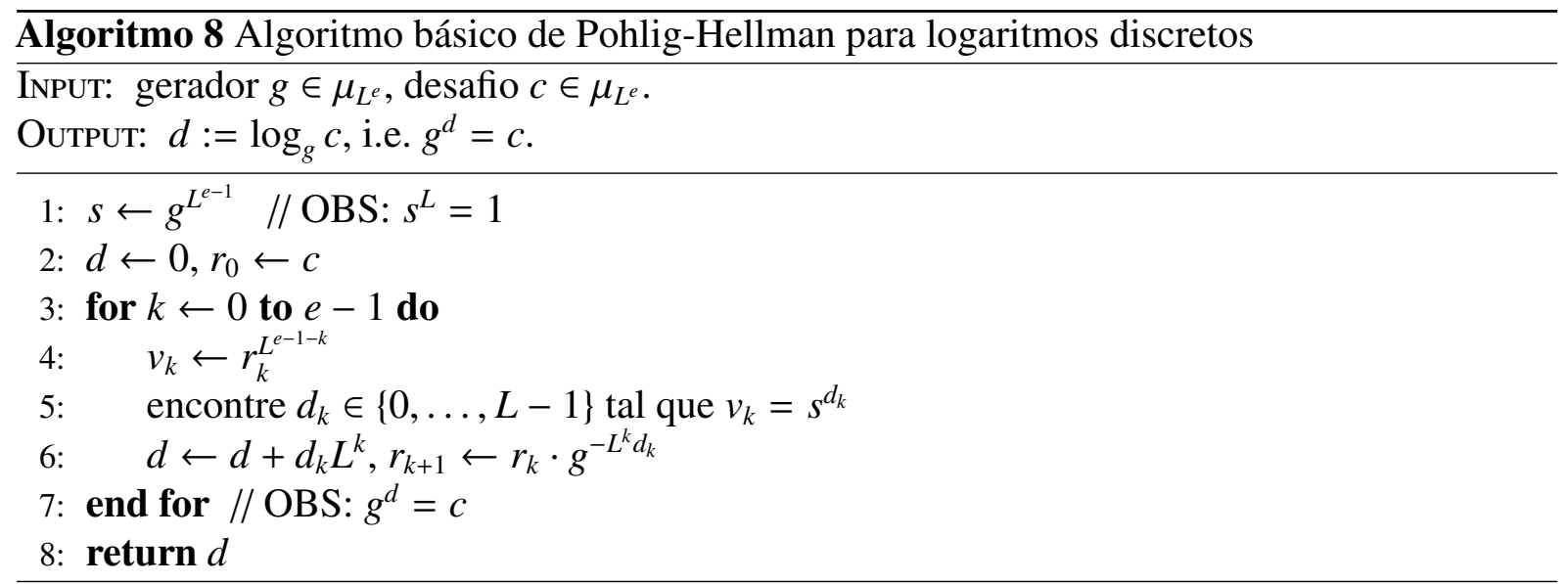

Assumindo que $g^{-L^{k}}$ é pré-computado e armazenado para todo $k$ como um subproduto do cálculo de $s$, a estratégia ingênua para obter o logaritmo discreto requer computar repetidamente o exponencial $r_{k}^{L^{e-1-k}}$ ao custo de elevar $e-1-k$ vezes a $L$, depois resolvendo uma pequena instância do logaritmo discreto em um subgrupo de ordem $L$ para obter o dígito $L$-ário, então limpando aquele dígito no expoente de $r_{k}$ a um custo que não exceda $L$ multiplicações para obter $r_{k+1}$. O custo total é, portanto, $O\left(e^{2}\right)$.

Acontece que essa estratégia está longe de ser ideal, como apontado por Shoup (SHOUP, 2005, Capítulo 11). A tarefa crucial é obter a sequência $r_{0}^{L^{e-1}}, r_{1}^{L^{e-2}}, r_{2}^{L^{e-3}}, \ldots, r_{e-1}^{L^{0}}$ nesta ordem, uma vez que cada $r_{k}$ depende do anterior. Pode-se visualizar essa tarefa usando um grafo acíclico direcionado $\Delta$ notavelmente semelhante ao grafo $T_{n}$ de De Feo et al., o qual eles chamam de "triângulo equilátero discreto", que modela a construção de isogenias de grau suave (DeFEO; JAO; PLÛT, 2014, Seção 4.2.2).

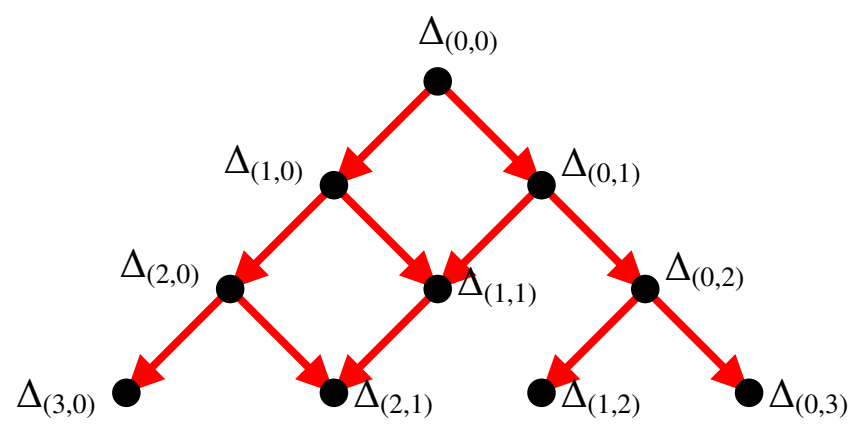

Figura 15 - Exemplo de um triângulo equilátero discreto $\Delta$.

Nesse caso, o conjunto de vértices é $\left\{\Delta_{j, k} \mid j+k \leq e-1\right\}$ onde $\Delta_{j, k}:=r_{k}^{L^{j}}$. Cada vértice possui ou duas arestas de saída para baixo ou nenhuma aresta. Vértices $\Delta_{j, k} \operatorname{com} j+k>e-1$ têm duas arestas: uma aresta esquerda $\Delta_{j, k} \rightarrow \Delta_{j+1, k}$ que modela a exponenciação do vértice 
de origem à $L$-ésima potência para produzir o vértice de destino, $r_{k}^{L^{j+1}} \leftarrow\left(r_{k}^{L^{j}}\right)^{L}$, e uma aresta à direita $\Delta_{j, k} \rightarrow \Delta_{j, k+1}$ que modela a limpeza do $(j+k)$-ésimo dígito do expoente do vértice de origem, $r_{k+1}^{L^{j}} \leftarrow r_{k}^{L^{j}} \cdot g^{-L^{(j+k)} d_{k}}$. Vértices $\Delta_{j, k} \operatorname{com} j+k=e-1$ são folhas, uma vez que não possuem arestas de saída.

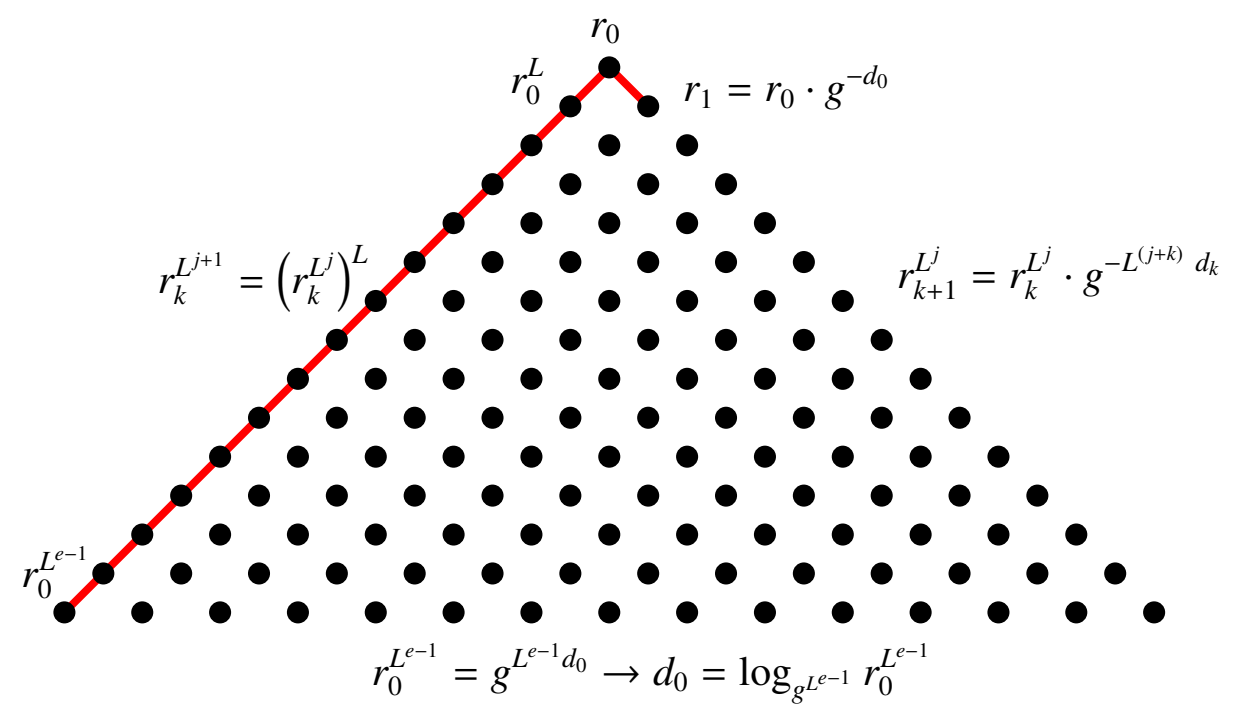

Figura 16 - Caminhar para a direita em $\Delta$ equivale a uma exponenciação e para a direita, remover $\mathrm{o}(j+k)$-ésimo dígito do expoente do vértice de origem.

De Feo et al. (DeFEO; JAO; PLÛT, 2014, Equação 5) descrevem um algoritmo de programação dinâmica com complexidade $O\left(e^{2}\right)$ que calcula o custo de uma sub-árvore de $\Delta$ ótima com raiz em $\Delta_{00}$ e cobrindo todas as folhas. Se o custo de percorrer uma aresta à direita ou à esquerda for $p$ ou $q$ respectivamente, e o custo de uma sub-árvore ótima de $k$ arestas é $C_{p, q}(k)$, o algoritmo deles é baseado nas relações $C_{p, q}(1)=0$ e $C_{p, q}(k)=\min _{1 \leq j<k}\left(C_{p, q}(j)+C_{p, q}(k-j)+(k-j) p+j q\right)$ para $k>1$.

A abordagem de programação dinâmica ingênua é armazenar os valores de $C_{p, q}(k)$ para $k=1 \ldots e$, chamando a relação acima $k-1$ vezes em cada passo para encontrar o mínimo correspondente, por um total de $e(e-1) / 2$ chamadas, portanto, o custo de $O\left(e^{2}\right)$. No entanto, como $C_{p, q}(k)$ não possui um mínimo local diferente do mínimo global único (ou duas cópias adjacentes e equivalentes ao mínimo global no pior caso), pode-se encontrar o mínimo com uma variante da busca binária que compara dois valores consecutivos próximos à metade do intervalo de busca $[1 \ldots k-1]$ e, em seguida, divide o intervalo no meio. Isso produz o Algoritmo 9, de complexidade $O(e \log e)$, que calcula $C_{p, q}(k)$ e a estrutura da estratégia de percurso ideal, armazenando os valores de $j$ acima que atingem o mínimo em cada etapa. 


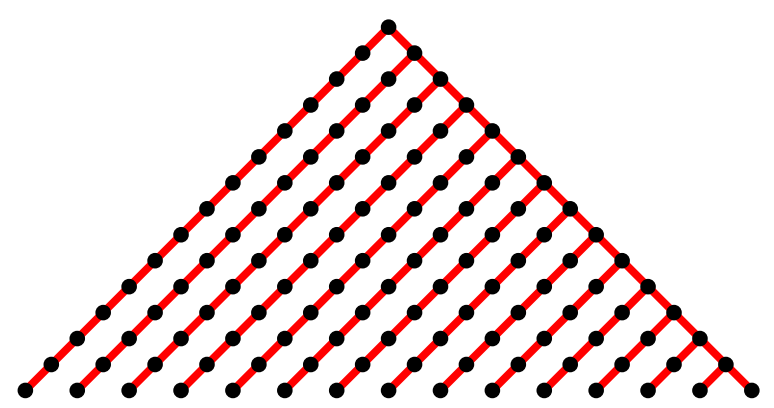

Figura 17 - Exemplo de caminho em $\Delta$ traçado pela estratégia ingênua.

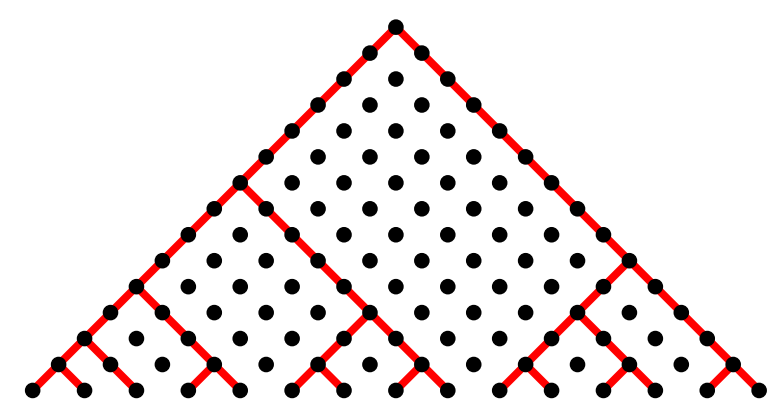

Figura 18 - Exemplo de caminho em $\Delta$ traçado pela estratégia ótima.

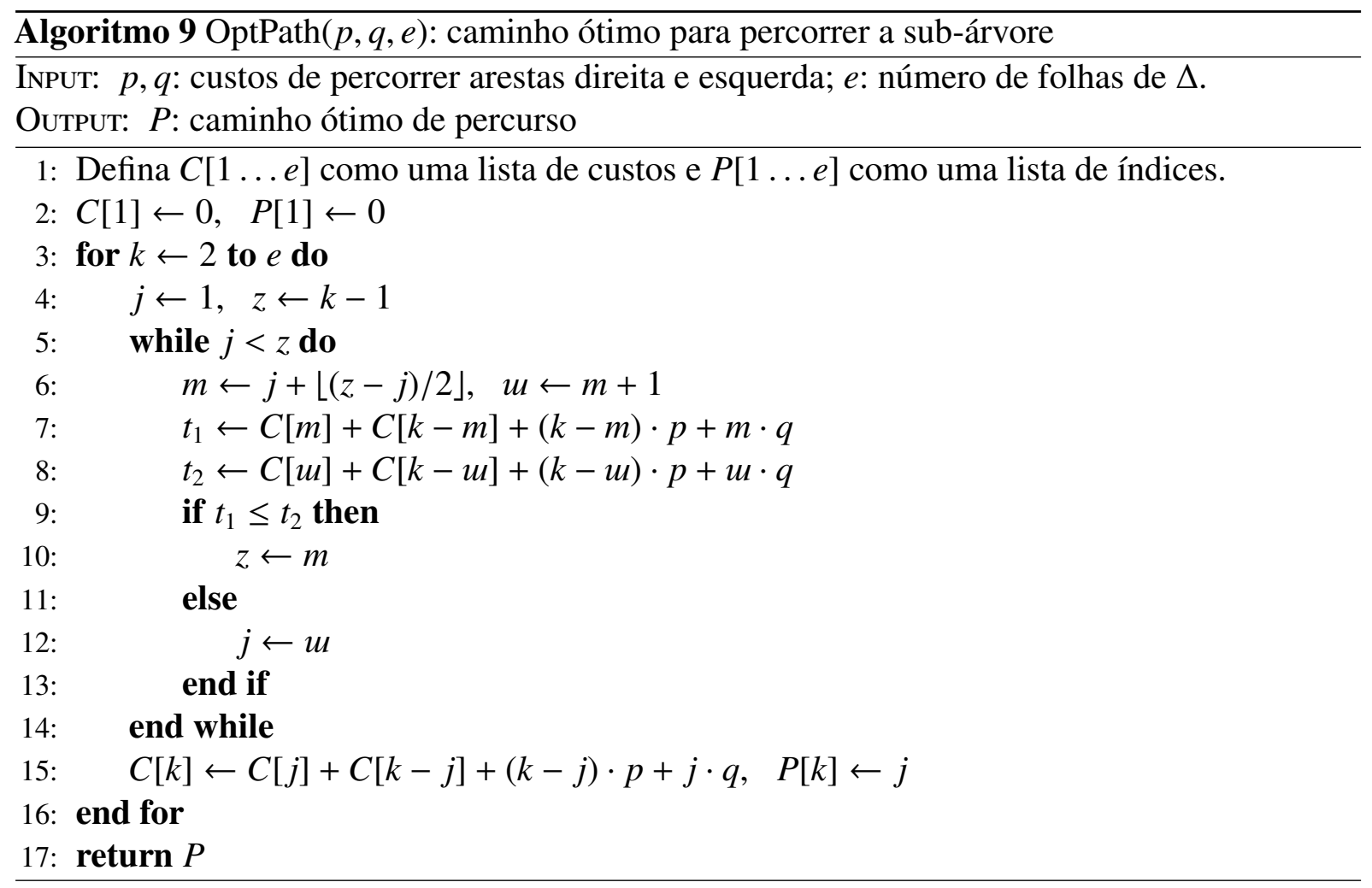

\subsubsection{O custo do cálculo do logaritmo discreto}

O custo de uma estratégia ótima depende dos custos individuais de atravessar uma aresta esquerda e uma direita. Mostra-se agora que, por causa da proposta de técnica de decomposição reversa, o custo total do cálculo do logaritmo discreto é drasticamente reduzido. Lembre-se que $L:=\ell^{w}$, onde $\ell$ é um primo pequeno e $w>0$ é um inteiro pequeno. Percorrer a aresta esquerda representa o cálculo de $r_{k}^{L^{j+1}} \leftarrow\left(r_{k}^{L^{j}}\right)^{L}$ a um custo $w \mathbf{S} \approx 1.6 w \mathbf{m}$ no caso binário e $w \mathbf{C}=w(2 \mathbf{m}+1 \mathbf{s}) \approx 2.8 w \mathbf{m}$ no caso ternário.

Percorrer a aresta direita representa o cálculo $r_{k+1}^{L^{j}} \leftarrow r_{k}^{L^{j}} \cdot g^{-L^{(j+k)} d_{k}}$, que pode ser realizado por meio de uma busca em tabela $r_{k+1}^{L^{j}} \leftarrow r_{k}^{L^{j}} \cdot T[j+k]\left[d_{k}\right]$ onde $T[u][d]:=g^{-L^{u} \cdot d}$. Como $j+k \leq e-1$, o tamanho da tabela é de $e \cdot L$ elementos do corpo. Isso permite um trade-off (relação de compromisso entre espaço e desempenho) ao calcular logaritmos discretos em $\mu_{\ell^{m}}$ 


\begin{tabular}{|c|c|c|c|c|}
\hline grupo & (COSTELLO et al., 2017) & este, $w=1$ (razão) & este, $w=3$ (razão) & este, $w=6$ (razão) \\
\hline$\mu_{2^{372}}$ & $8271.6 \mathbf{m}$ & $4958.4 \mathbf{m}(0.60)$ & $3127.9 \mathbf{m}(0.39)$ & $2103.7 \mathbf{m}(0.25)$ \\
$\mu_{3^{239}}$ & $7999.2 \mathbf{m}$ & $4507.6 \mathbf{m}(0.56)$ & $2638.1 \mathbf{m}(0.33)$ & $1739.8 \mathbf{m}(0.22)$ \\
\hline
\end{tabular}

Tabela 5 - Custos do cálculo do logaritmo discreto (assumindo $\mathbf{s} \approx 0.8 \mathbf{m}$ )

com $w \mid m$, ou seja, calculando logaritmos discretos em $\mu_{\left(\ell^{w}\right)^{m / w}}$, que coincide com $\mu_{L^{e}}$ para $e=m / w$. Nesse caso, o tamanho da tabela pode ser escrito como $(m / w) \cdot \ell^{w}$ para mostrar mais claramente a dependência do tamanho desses parâmetros.

No entanto, não mais do que uma única multiplicação é incorrida independentemente de $\ell, e$, ou $w$, ou seja, $1 \mathbf{M} \approx 3 \mathbf{m}$. Quando $w$ é muito pequeno, evitar a multiplicação por $d_{k}=1$ reduz visivelmente o tempo de execução e requer menos entradas na tabela. Além disso, a tabela é fixa com a técnica de decomposição reversa de base, porque $g=\mathrm{e}\left(P_{\mathrm{B}}, Q_{\mathrm{B}}\right)^{\operatorname{deg} \phi_{\mathrm{A}}}$, ou $g=\mathrm{e}\left(P_{\mathrm{A}}, Q_{\mathrm{A}}\right)^{\operatorname{deg} \phi_{\mathrm{B}}}$, o que não acarreta em custos adicionais durante a construção da tabela em tempo de execução para cada chave recém-gerada. Mesmo as instâncias de logaritmo discretas simples nas folhas incorrem apenas em buscas $O(L)$ na mesma tabela, uma vez que $s^{d_{k}}=\overline{T[e-1]\left[d_{k}\right]}$.

O Algoritmo 10 resume a técnica proposta, combinando o algoritmo RDL do Shoup (SHOUP, 2005, Seção 11.2.3) com a estratégia ótima de divisão e conquista de De Feo et al. e a busca em tabela eficiente possível pela técnica de decomposição reversa de base. Seguindo a análise de Shoup como indicado, e assumindo que a estratégia ótima é aproximadamente balanceada (uma suposição razoável, de acordo com De Feo et al.), obtém-se um custo assintótico de $O(e \log e)$ multiplicações em $\mathbb{F}_{p}$. Observe que, ao contrário de Shoup, o algoritmo baby-step giant-step não é necessário para calcular os logaritmos elementares, já que nesse caso eles correspondem a grupos de ordem 2 ou 3.

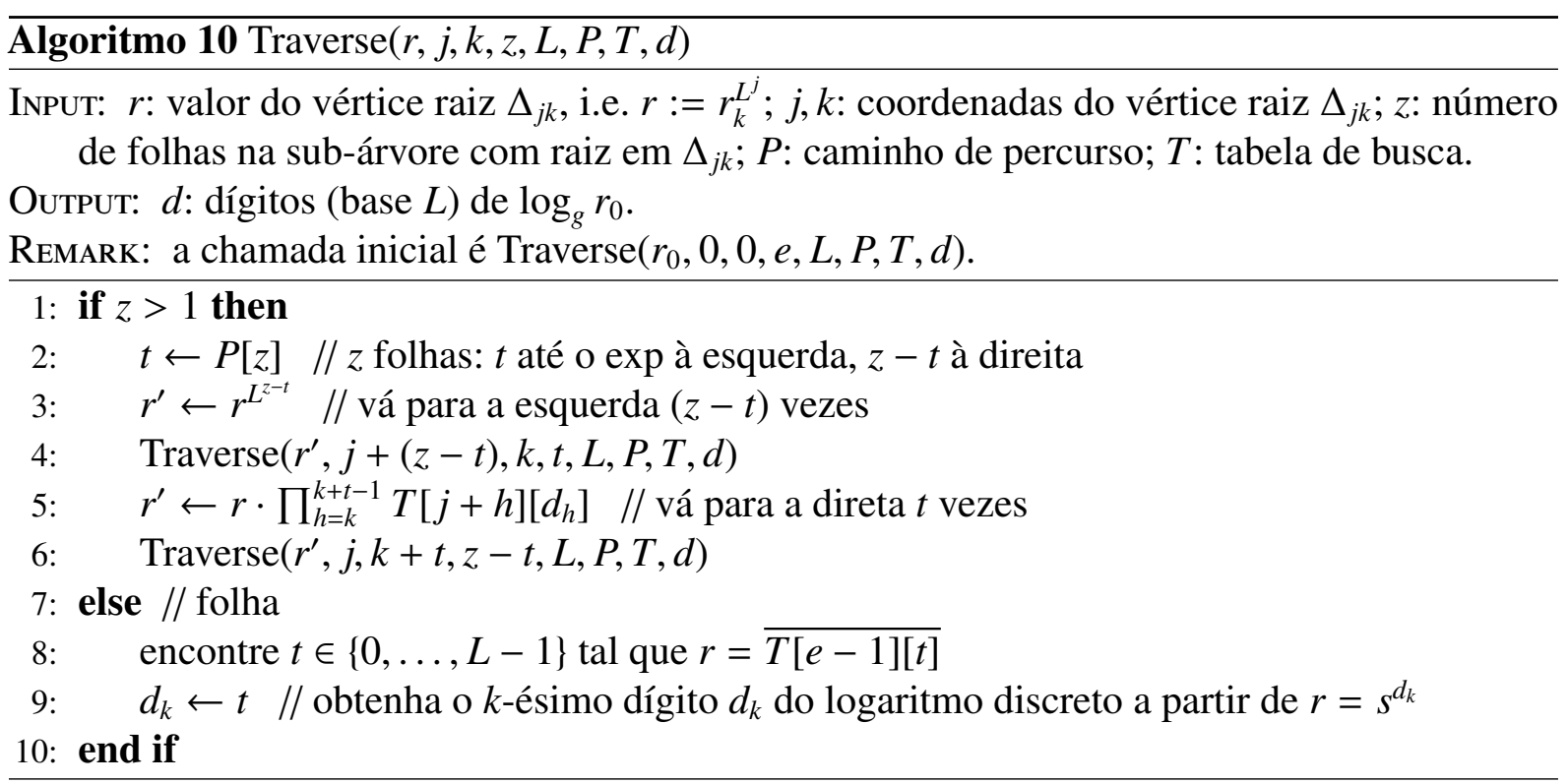


As melhorias resultantes são substanciais. Para logaritmos discretos em $\mu_{2^{372}}$, o custo ótimo é $\approx 4958.4 \mathbf{m}$ para $w=1, \approx 3127.9 \mathbf{m}$ para $w=3$, e $\approx 2103.7 \mathbf{m}$ para $w=6$. Para logaritmos discretos em $\mu_{3^{239}}$, o custo ótimo é $\approx 4507.6 \mathbf{m}$ para $w=1, \approx 2638.1 \mathbf{m}$ para $w=3$, e $\approx 1739.8 \mathbf{m}$ para $w=6$.

Trade-offs também são possíveis. Em vez de uma matriz de tamanho $(m / w) \cdot \ell^{w}$, a tabela de busca pode ser restringida a uma simples lista $T_{1}[u]:=g^{-\ell^{w u}}$ de $(m / w)$ entradas, ao calcular $T_{1}[u]^{d}=g^{-\ell^{w u} \cdot d}$ sob demanda usando uma cadeia de multiplicações ótima para exponenciações ciclotômicas. Por exemplo, logaritmos discretos em $\mu_{2^{372}}$ com $w=3$ exigiriam uma tabela de tamanho 124 com um custo médio de $\approx 4453.9 \mathrm{~m}$. Por comparação, os melhores resultados relatados em (COSTELLO et al., 2017, Seção 5) são 2150m + 7652s $\approx 8271.6 \mathbf{m}$ para logaritmos discretos $\mu_{2^{372}}$ e $5320 \mathbf{m}+3349 \mathbf{s} \approx 7999.2 \mathbf{m}$ para logaritmos discretos $\mu_{3^{239}}$, ambos com $w=3, \mathrm{o}$ que é ótimo nessa técnica; $\mathrm{O}$ aumento de $w$ é observado para causar um aumento de custo.

A Tabela 5 resume os ganhos que a técnica proposta torna possível e os compara com os resultados de (COSTELLO et al., 2017), tanto em termos do número bruto de multiplicações no corpo base quanto da relação entre os resultados obtidos e o deles. É válido lembrar que nenhuma preocupação de com relação a ataques de canal colateral surge dessa técnica, uma vez que todas as informações envolvidas no processamento são públicas.

\subsubsection{Pohlig-Hellman aprimorado para $w$ genérico}

Reescrevendo o algoritmo de Pohlig-Hellman 8 para calcular logaritmos discretos em $\mu_{\ell^{m}}$ e representando o expoente $d$ na base $\ell^{w} \operatorname{com} d=\sum_{i=0}^{[m / w\rceil} d_{i} \ell^{w i}$, nos permite recuperar mais bits do expoente por dígito de forma simples quando $w \mid m$. por outro lado, o caso onde $w \nmid m$ requer uma atenção especial. O pior caso ocorre quando $m$ é um número primo e portanto não-divisível por $1<w<m$. Esse é o caso do primo do SIDH em na implementação, onde o logarimo discreto ternário é definido sob um subgrupo de ordem $3^{239}$, onde 239 é primo.

Um algoritmo que lida com os casos em que $w \nmid m$ será deduzido a seguir. Denote o desafio por $r_{0}:=g^{d} \in \mu_{\ell^{m}}$ onde $d=\sum_{i=0}^{\lceil m / w\rceil-1} d_{i} \ell^{w i}$ e seja $r_{0}$ a raiz $\Delta_{00}:=r_{0}$ do grafo $\Delta$. Note que aqui, $\Delta$ é um triângulo discreto equilátero de lado $s=\lceil m / w\rceil$ vértices. Na versão usual com expoente divisível, percorrer uma aresta à esquerda é equivalente a elevar o elemento atual $\Delta_{j k}$ à $\ell^{w}$-ésima potência, i.e., $r_{k}^{\rho j w+w} \leftarrow r_{k}^{\ell j w}$. Quando $w \mid m$, o valor $r_{k}$ é elevado para a $\ell^{w}$-ésima potência $(s-k)-1$ vezes para que o elemento resultante da folha $\Delta_{j, k}=r_{k}^{\ell^{(s-k-1) w}}$ for $j+k=s-1$ pertença ao subgrupo de ordem $\ell^{w}$. O $k$-ésimo dígito correspondente é então recuperado simplesmente resolvendo-se o logaritmo $d_{k}:=\log _{g^{m-w}} \Delta_{j, k}$.

Quando $w \nmid m$, uma observação crucial é que, quando o elemento $r_{k}$ é elevado à $\ell^{(s-k-1) w_{-}}$ ésima potência, o elemento correspondente da folha $\Delta_{j, k}$ pertence ao subgrupo de ordem $\ell^{m \bmod w}$ 
(em vez de $\ell^{w}$ ) pois por construção $r_{k}$ possui ordem $\ell^{m-k w} \mathrm{e}$

$$
\begin{aligned}
\Delta_{j, k} & =r_{k}^{\ell^{(s-k-1) w}} \\
& =r_{k}^{\ell^{s w-k w-w}} \\
& =r_{k}^{\ell^{(m-m \bmod w+w)-k w-w}} \\
& =r_{k}^{\ell^{m-k w-m \bmod w}} .
\end{aligned}
$$

Portanto, resolver esse logaritmo discreto menor nos permitiria apenas recuperar uma informação parcial do dígito e não seria possível recuperar o a totalidade do expoente ao fim do processo. para corrigir as ordens dos elementos das folhas, algumas modificações na estratégia de percurso ótimo (Algoritmo 10) são necessárias. A ideia principal para se obter folhas com a ordem desejada $\ell^{w}$ consiste de modificar o primeiro percurso do vértice esquerdo para cada vértice mais a direita $\Delta_{0, k}$. Os primeiros percursos à esquerda serão dados por $\Delta_{1, k} \leftarrow \Delta_{0, k}^{\ell^{m} \bmod w} \mathrm{e}$ $\Delta_{j+1, k} \leftarrow \Delta_{j, k}^{\ell^{w}}$ para $j>0$, ou equivalentemente, o percurso de $\Delta_{j, k}$ para $\Delta_{j+1, k}$ é definido como $r_{k}^{\ell^{m \bmod w+j w}} \leftarrow r_{k}^{\ell^{m \bmod w+(j-1) w}}$

Isso nos leva à seguinte redefinição de $\Delta_{j, k}$. Seja o conjunto de vértices $\left\{\Delta_{j, k} \mid j+k \leq\right.$ $\lceil m / w\rceil-1\}$, então $\Delta_{j, k}:=r_{k}$ para $j=0$ e $\Delta_{j, k}:=r_{k}^{\ell^{m} \bmod w+(j-1) w}$ para $j>0$.

Ao aplicar esta modificação, todas as folhas terão ordem $\ell^{w}$ exceto a da extrema direita, que terá ordem $\ell^{m \bmod w}$.

Os percursos das arestas à direita, responsáveis por remover os dígitos do expoente, também são modificados. São dados por $\Delta_{j, k+1} \leftarrow \Delta_{j, k} \cdot g^{-d_{k} \cdot \ell^{m \bmod w+(j-1) w}}$. Como os vértices que não estão na extrema direita $\Delta_{j, k}$ são calculados como $\Delta_{0, k}^{\ell^{m} \text { mod } w+(j-1) w}$, os dígitos no expoente terão coeficientes deslocados por $\ell^{w-m \bmod w}$, e portanto uma tabela de remoção de dígitos adequada pode ser definida $T_{2}[0]\left[d_{i}\right]:=g^{-d_{i}}$ para $0 \leq d_{i}<\ell^{w}-1$ e $T_{2}[u]\left[d_{i}\right]:=g^{-d_{i} \cdot \ell^{m \bmod w+(u-1) w}}$ para $0<u<s$. Esta tabela também é usada para calcular logaritmos discretos nas folhas $0,1, \cdots, s-2$ já que sua última linha terá elementos de ordem $\ell^{w}$. Uma tabela distinta será necessária para lidar com percursos de arestas direitas que não estão na extrema direita e cálculo dos logaritmos discretos através das arestas na extrema direita $\Delta_{0, k}$. Essa tabela é definida por $T_{1}[v]\left[d_{i}\right]:=g^{-d_{i} \cdot \ell^{v w}}$ para $0 \leq v<s$.

O Algoritmo 11, detalha o procedimento completo para calcular a versão do PohligHellman para $w$ genérico combinado com a estratégia de percurso ótimo.

O caminho ótimo $P$ é obtido invocando $\operatorname{OptPath}(w \cdot p, q,\lceil m / w\rceil)$ onde $p$ é o custo de elevar a $\ell$, e $q$ é o custo de uma multiplicação na extensão quadrática do corpo. 


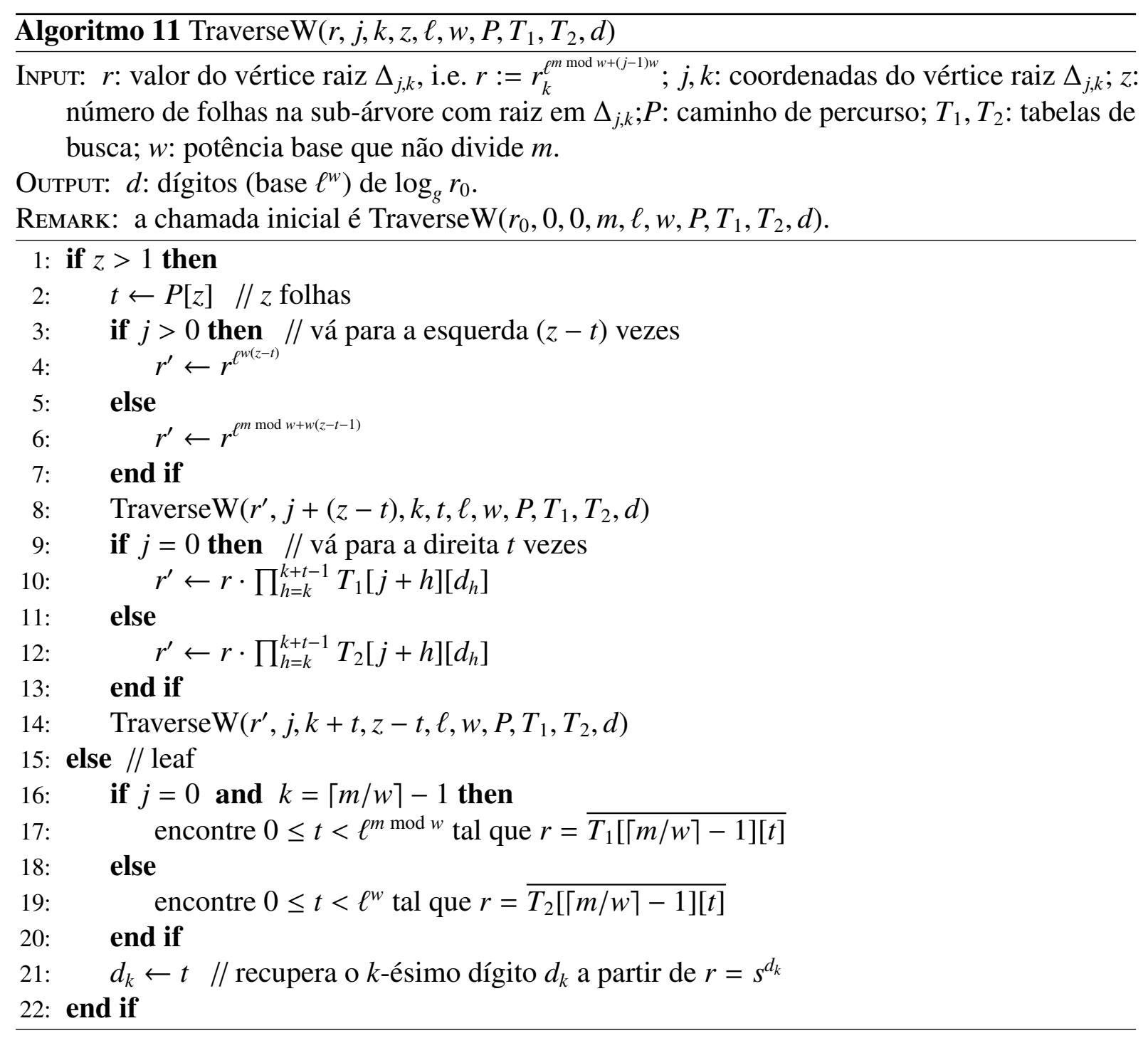




\section{Resultados práticos}

Foi feita uma implementação completa em SageMath como prova de conceito, dada a flexibilidade e facilidade para manipular as estruturas algébricas em que as propostas se apoiam mas não diretamente relacionados às contribuições desta pesquisa (por exemplo, geração de curvas elípticas isógenas). Essa implementação foi traduzida para Python puro visando torná-la independente das primitivas oferecidas pela linguagem anterior, além de auto-contida o suficiente para servir como referência ao implementar os algoritmos em linguagem mais performática.

Uma outra implementação, em linguagem C, foi realizada por outro membro da equipe de pesquisa (a saber, Geovandro C. C. F. Pereira). A implementação apoia-se na biblioteca SIDH/SIKE da Microsoft Research para os mesmos propósitos, e visa a facilitar uma comparação mais precisa com estado da arte em criptografia baseada em isogenias em plataformas realísticas.

Após a publicação das técnicas aqui relatadas, elas foram incorporadas à biblioteca SIDH/SIKE da Microsoft Research (Microsoft SIDH team, 2017). Originalmente, essa biblioteca havia sido projetada para o número primo específico $p=2^{372} 3^{239}-1$ de tamanho $\log p=751$ bits. Atualmente, outros tamanhos são igualmente suportados. Este documento, porém, apenas relata os resultados experimentais daquele valor original para facilitar a comparação com valores publicados na literatura, também anteriores à generalização de parâmetro acima.

As técnicas de compressão e descompressão de chave aprimoradas foram implementadas em cima da biblioteca SIDH/SIKE em linguagem C (Microsoft SIDH team, 2017) para disponibilizar uma troca de chaves totalmente flexível. As funções anteriores de (des)compressão foram deixadas na nova versão para que os experimentos possam ser replicados para comparações.

Como apenas informações públicas são processadas (compressão e descompressão de chaves públicas), os ataques de canal lateral não são um problema. Isso permitiu, por exemplo, a adoção de uma versão simples e rápida do algoritmo de Euclides estendido.

A metodologia de benchmark (avaliação comparativa por desempenho) consistiu em tomar a média das operações individuais de (des)compressão de chaves, como geração de base, cálculo de logaritmos discretos e emparelhamento em 20 mil execuções para entradas aleatórias e 800 execuções para operações mais caras como (des)compressão de chave. Os resultados expressam-se em Megaciclos (Mciclos) e foram realizados em uma máquina com um processador Intel Core i5-6267U de $2.9 \mathrm{GHz}$. A biblioteca foi compilada pelo compilador clang, flags -03 e $\mathbf{s}=\mathbf{m}$ nessa implementação.

A curva pública inicial é a curva supersingular usual $E_{0}: y^{2}=x^{3}+x$ definida sob $\mathbb{F}_{p^{2}}$ onde $p=2^{372} 3^{239}-1$. Vale ressaltar que, antes de aplicar as técnicas propostas de (des)compressão, a biblioteca SIDH v2.0 foi primeiramente modificada para realizar a geração de chaves da 
Alice com ambos pontos $P_{\mathrm{A}}$ e $Q_{\mathrm{A}}$ definidos na extensão $E_{0}\left(\mathbb{F}_{p^{2}}\right) \backslash E_{0}\left(\mathbb{F}_{p}\right)$ em vez de definir $P_{\mathrm{A}}$ no corpo base como sugerido em (COSTELLO et al., 2017). A abordagem em (COSTELLO et al., 2017) inicia com o ponto $P_{\mathrm{A}}=(x, y) \in E_{0}\left(\mathbb{F}_{p}\right)$ sob o corpo base e então aplica o mapa de distorção $\tau$ para obter um ponto linearmente independente $Q_{\mathrm{A}}=\tau\left(P_{\mathrm{A}}\right)=(-x, i y)$ no grupo de traço zero. Essa otimização não pode ser combinada com as técnicas propostas pois utilizar mapas de distorção em torsões binárias nos dá apenas bases $\left\langle P_{\mathrm{A}}, \tau\left(P_{\mathrm{A}}\right)\right\rangle=E_{0}\left[2^{m-1}\right]$ de um grupo menor de ordem $2^{2(m-1)}$, e nesse caso as imagens de $P_{\mathrm{A}}$ e $Q_{\mathrm{A}}=\tau\left(P_{\mathrm{A}}\right)$ sob a isogenia de Bob consequentemente geram uma torção menor, i.e. $\left\langle\phi_{\mathrm{B}}\left(P_{\mathrm{A}}\right), \phi_{\mathrm{B}}\left(Q_{\mathrm{A}}\right)\right\rangle=E_{\mathrm{B}}\left[2^{m-1}\right]$.

Em particular, a técnica de decomposição reversa de base juntamente com a base emaranhada não funcionaria uma vez que a base emaranhada gera um grupo de $2^{m}$-torção, e a base não pode ser convertida para uma base de menor torção, i.e. a matriz de mudança de base na equação (4.3) não existiria. Portanto, os pontos

$$
P_{\mathrm{A}}:=3^{239} \cdot\left(5+i, \sqrt{(5+i)^{3}+5+i}\right) \in E_{0}\left(\mathbb{F}_{p^{2}}\right) \backslash E_{0}\left(\mathbb{F}_{p}\right)
$$

e $Q_{\mathrm{A}}:=\tau\left(P_{\mathrm{A}}\right) \in E_{0}\left(\mathbb{F}_{p^{2}}\right) \backslash E_{0}\left(\mathbb{F}_{p}\right)$ são selecionados. Pontos $P_{\mathrm{B}}$ e $Q_{\mathrm{B}}$ são os dados em (COSTELLO et al., 2017) pois para torsões de $\ell^{n} \operatorname{com} \ell$ ímpar, mapas de distorção geram o grupo completo $E_{0}\left[\ell^{n}\right]$ e $P_{\mathrm{B}}$ pode ser mantido no corpo base. Para os logaritmos discretos definimos $w=2$ para o caso binário e $w=6$ para o ternário. Neste último caso, a decisão foi tomada para compensar o fato de que ainda não se tem uma base emaranhada para o caso ternário.

Os experimentos para a geração de base de $3^{n}$-torção são apresentados na Tabela 6 , onde as três melhorias obtidas em relação ao 3-descenso anterior implementado na biblioteca SIDH v2.0 são mostradas. Elas consistem do método ingênuo (contudo mais rápido do que o 3-descenso), ingênuo + Elligator compartilhado e o ingênuo + Elligator compartilhado com a técnica de geração de base sem teste de independência linear.

\begin{tabular}{lccc}
\hline técnica & referência & Mciclos & razão \\
\hline 3-descenso & SIDH v2.0 (COSTELLO et al., 2017) & 19.98 & - \\
geração de base ingênua & este trabalho & 17.33 & $\mathbf{1 . 2}$ \\
Elligator compartilhado & este trabalho & 13.63 & $\mathbf{1 . 5}$ \\
Ell. compart. + sem ver. LI & este trabalho & 7.26 & $\mathbf{2 . 8}$ \\
\hline
\end{tabular}

Tabela 6 - Benchmark da geração da base de $3^{\mathbf{n}}$-torção.

Quanto à geração de base de $2^{m}$-torção em conjunto com o Elligator compartilhado para descompressão, os resultados de benchmark mostram que a barreira dos 1 Mciclos pode ser quebrada pela primeira vez, exigindo apenas 0.83 Mciclos para gerar a base que apresenta um ganho substancial contra o 2-descenso anterior, com custo de 23.77 Mciclos na biblioteca SIDH v2.0. Observe que nesse caso a verificação de independência linear é dada de forma implícita (devido ao Teorema 30). Os resultados são mostrados na Tabela 7. 


\begin{tabular}{lccc}
\hline técnica & referência & Mciclos & razão \\
\hline 2-descenso & SIDH v2.0 (COSTELLO et al., 2017) & 23.77 & - \\
base emaranhada & este trabalho & 1.60 & $\mathbf{1 4 . 9}$ \\
base emaranhada + ell. compart. & este trabalho & 0.83 & $\mathbf{2 8 . 6}$ \\
\hline
\end{tabular}

Tabela 7 - Benchmark da geração da base de $2^{\mathbf{m}}$-torção

A Tabela 8 resume os resultados experimentais para as operações de alto nível de (des)compressão de chaves. As pequenas diferenças entre as estimativas teóricas e os resultados práticos são basicamente devido ao custo de elevar ao quadrado no corpo base $\mathbb{F}_{p}$ que é implementado na biblioteca SIDH v2.0 por reuso de multiplicação modular em vez de adotar uma técnica otimizada de Montgomery para elevar ao quadrado. Nesse caso, elevar ao quadrado no corpo finito não custa entre 0.6 e $0.8 \mathbf{m}$ apesar de ser possível de ser obtido na prática.

\begin{tabular}{lccccccc} 
& \multicolumn{3}{c}{$\mathbf{2}^{\boldsymbol{m}}$-torção $(\boldsymbol{w}=\mathbf{2})$} & & \multicolumn{3}{c}{$\mathbf{3}^{n}$-torção $(\boldsymbol{w}=\mathbf{6})$} \\
\cline { 2 - 3 } operação & SIDH v2.0 & este trabalho & razão & & SIDH v2.0* & este trabalho & razão \\
\hline um logaritmo discreto & 5.88 & 2.57 & $\mathbf{2 . 3}$ & & 4.71 & 1.17 & $\mathbf{4 . 0}$ \\
fase de emparelhamento & 33.23 & 25.37 & $\mathbf{1 . 3}$ & & 37.72 & 29.04 & $\mathbf{1 . 3}$ \\
\hline compressão & 75.49 & 37.07 & $\mathbf{2 . 0}$ & & 79.33 & 54.14 & $\mathbf{1 . 5}$ \\
descompressão & 28.76 & 8.97 & $\mathbf{3 . 2}$ & & 25.95 & 12.91 & $\mathbf{2 . 0}$ \\
\hline Note que a descompressão incorpora a otimização do Elligator compartilhado. & *SIDH v2.0" refere-se aos resultados relatados em (COSTELLO et al., 2017). & &
\end{tabular}

Tabela 8 - Resultados do obtidos por por etapa do protocolo.

A Tabela 9 mostra o impacto dos algoritmos de (des)compressão no SIKE. Incorporar a compressão e descompressão de chaves no SIKE é simples; a compressão é feita apenas durante a geração de chaves, que é baseada na $2^{m}$-torção das curvas. A vantagem da escolha de $2^{m}$-torção é que tem-se algoritmos muito mais rápidos como a geração da base emaranhada. A descompressão é feita imediatamente antes do encapsulamento. Observe que não há (des)compressão no caso de $3^{n}$-torção. Um pequeno overhead para o primo p964 se dá pelo uso do mesmo algoritmo de compressão juntamente com uma implementação não otimizada (única opção disponível para esse primo). Por exemplo, para p964 o cálculo da isogenia é o algoritmo baseado em multiplicação com complexidade quadrática enquanto para os outros primos, é quase linear.

\begin{tabular}{lrccccc} 
primo & SIKE ger. & SIKE encaps. & $2^{m}$ compr. & $2^{m}$ descompr. & overhead na ger. & overhead no encaps. \\
\hline p503 & $9.1 *$ & $15.1 *$ & $10.2(w=6) * *$ & $3.06 * *$ & $112 \%$ & $20 \%$ \\
p751 & $27.5 *$ & $44.5 *$ & $31.7(w=6) *$ & $8.97 *$ & $115 \%$ & $20 \%$ \\
p964 & $13570.8 *$ & $19406.8 *$ & $294.7(w=6) * *$ & $84.45 * *$ & $2.2 \%$ & $0.4 \%$ \\
\hline
\end{tabular}

** Tempos de execução estimados baseados na contagem de operações em $\mathbb{F}_{p}$.

Tabela 9 - Impactos dos algoritmos de (des)compressão no SIKE. 



\section{Conclusão}

Cada um dos resultados obtidos, a saber, construção das bases de torção, bases emaranhadas, Elligator compartilhado, estratégia ótima no cálculo de logaritmos discretos, otimizações no cálculo de emparelhamento e decomposição de base reversa, não tiveram uma contribuição significativa se tratados individualmente. Contudo, quando tratados em conjunto, esses resultados chegaram a obter ganhos de até $\approx 28 \times$ vezes em relação ao trabalho anterior.

Este trabalho produziu dois artigos, o primeiro aceito na conferência internacional Crypto (ZANON et al., 2018) e o segundo, aceito na revista científica IEEE Transactions on Computers, (ZANON et al., 2019). Os resultados serviram de base para os trabalhos de Geovandro Pereira, Javad Doliskani e David Jao (PEREIRA; DOLISKANI; JAO, 2020), bem como de Michael Naehrig e Renes Joost (NAEHRIG; RENES, 2019), e possibilitaram que o uso de compressão de chaves se tornasse uma opção do esquema SIKE, atualmente um dos finalistas na competição de padronização do NIST.

Em vista disso, conclui-se que o objetivo principal deste trabalho foi alcançado.

\subsection{Sugestões para pesquisas futuras}

Apesar de sua primeira publicação ter sido em 1997, criptossistemas baseados em isogenias ainda são muito recentes se comparados às demais alternativas, e por isso ainda há muito o que ser explorado, seja de forma prática por meio de implementações otimizadas, seja de forma teórica por meio de melhorias em algoritmos e provas de segurança.

Considerando apenas o cenário que envolvem técnicas de (des)compressão de chaves do SIDH, fica evidente que ainda existem gargalos passíveis de serem otimizados, em particular, no caso ternário onde os ganhos obtidos não foram tão substanciais quanto do caso binário.

Além disso, ainda há outros criptossistemas recentes baseados em isogenias como CSIDH (CASTRYCK et al., 2018), SeaSign (FEO; GALBRAITH, 2019) e CSI-FiSh (BEULLENS; KLEINJUNG; VERCAUTEREN, 2019) e que, em uma investigação futura, talvez revelem novas oportunidades de otimização nos tempos de processamento ou ocupação de banda. 



\section{Referências}

ALKIM, E.; DUCAS, L.; PöPPELMANN, T.; SCHWABE, P. Post-quantum key exchange: A new hope. In: Proceedings of the 25th USENIX Conference on Security Symposium. USA: USENIX Association, 2016. (SEC'16), p. 327-343. ISBN 9781931971324. Citado na página 16.

ARAGON, N.; BARRETO, P. S. L. M.; BETTAIEB, S.; BIDOUX, L.; BLAZY, O.; DENEUVILLE, J.-C.; GABORIT, P.; GUERON, S.; GUNEYSU, T.; MELCHOR, C. A.; MISOCZKI, R.; PERSICHETTI, E.; SENDRIER, N.; TILLICH, J.-P.; ZÉMOR, G. BIKE: Bit Flipping Key Encapsulation. 2017. Submission to the NIST post quantum standardization process. Disponível em: <https://hal.archives-ouvertes.fr/hal-01671903>. Citado na página 16.

AZARDERAKHSH, R.; FISHBEIN, D.; JAO, D. Efficient Implementations of A QuantumResistant Key-Exchange Protocol on Embedded systems. 2014. Citado 2 vezes nas páginas 21 e 23.

AZARDERAKHSH, R.; JAO, D.; KALACH, K.; KOZIEL, B.; LEONARDI, C. Key compression for isogeny-based cryptosystems. In: ACM. Proceedings of the 3rd ACM International Workshop on ASIA Public-Key Cryptography. Abu Dhabi, EAU, 2016. p. 1-10. Citado 4 vezes nas páginas 16, 23, 51 e 54.

BAHAJJI, Z. A. Indexing HTTPS pages by default. 2015. <https://webmasters.googleblog.com/ 2015/12/indexing-https-pages-by-default.html>. Acessado em 4 de agosto de 2019. Citado na página 15.

BARRETO, P. S. L. M. Curvas Elípticas e Criptografia: Conceitos e Algoritmos. 1999. Relatório técnico. Nenhuma citação no texto.

BARRETO, P. S. L. M.; KIM, H. Y.; LYNN, B.; SCOTT, M. Efficient algorithms for pairing-based cryptosystems. In: Advances in Cryptology - Crypto 2002. Santa Barbara (CA), USA: Springer, 2002. (Lecture Notes in Computer Science, 2442), p. 354-368. Citado na página 65.

BERNSTEIN, D. J.; DUIF, N.; LANGE, T.; SCHWABE, P.; YANG, B. High-speed high-security signatures. J. Cryptographic Engineering, v. 2, n. 2, p. 77-89, 2012. Disponível em: <http://dx.doi.org/10.1007/s13389-012-0027-1>. Citado na página 65.

BERNSTEIN, D. J.; HAMBURG, M.; KRASNOVA, A.; LANGE, T. Elligator: elliptic-curve points indistinguishable from uniform random strings. In: 2013 ACM SIGSAC Conference on Computer and Communications Security, CCS'13, Berlin, Germany, November 4-8, 2013. ACM, 2013. p. 967-980. Disponível em: <http://doi.acm.org/10.1145/2508859.2516734>. Citado 3 vezes nas páginas 23,42 e 56.

BERNSTEIN, D. J.; LANGE, T. Analysis and optimization of elliptic-curve single-scalar multiplication. In: Finite Fields and Applications: Proceedings of Fq8. Providence (RI), USA: American Mathematical Society, 2008. p. 1-18. Citado 2 vezes nas páginas 67 e 69. 
BEULLENS, W.; KLEINJUNG, T.; VERCAUTEREN, F. Csi-fish: Efficient isogeny based signatures through class group computations. In: . [S.1.: s.n.], 2019. p. 227-247. ISBN 978-3-030-34577-8. Citado na página 85.

BRAITHWAITE, M. Experimenting with Post-Quantum Cryptography. 2016. < https:

//security.googleblog.com/2016/07/experimenting-with-post-quantum.html>. Acessado em 5 de agosto de 2019. Citado na página 15.

CASTRYCK, W.; LANGE, T.; MARTINDALE, C.; PANNY, L.; RENES, J. Csidh: An efficient post-quantum commutative group action. In: PEYRIN, T.; GALBRAITH, S. (Ed.). Advances in Cryptology-ASIACRYPT 2018. Cham: Springer International Publishing, 2018. p. 395-427. Citado 2 vezes nas páginas 24 e 85.

CHEN, L.; JORDAN, S.; LIU, Y.-K.; MOODY, D.; PERALTA, R.; PERLNER, R.; SMITH-TONE, D. Report on Post-Quantum Cryptography. 2016. <https://csrc.nist.gov/ publications/detail/nistir/8105/final> . Acessado em 24 de novembro de 2020. Citado na página 22.

CHILDS, A. M.; JAO, D.; SOUKHAREV, V. Constructing elliptic curve isogenies in quantum subexponential time. Journal of Mathematical Cryptology, v. 8, n. 1, p. 1-29, 2014. Disponível em: <https://arxiv.org/abs/1012.4019>. Citado 2 vezes nas páginas 21 e 45.

CHUENGSATIANSUP, C.; NAEHRIG, M.; RIBARSKI, P.; SCHWABE, P. PandA: Pairings and arithmetic. In: CAO, Z.; ZHANG, F. (Ed.). Pairing-Based Cryptography - Pairing 2013. Cham: Springer International Publishing, 2014. p. 229-250. Citado na página 65.

COSTELLO, C. Supersingular isogeny key exchange for beginners. 2019. Cryptology ePrint Archive, Report 2019/1321. <https://eprint.iacr.org/2019/1321>. Nenhuma citação no texto.

COSTELLO, C.; HISIL, H. A simple and compact algorithm for SIDH with arbitrary degree isogenies. 2017. Cryptology ePrint Archive, Report 2017/504. <https://eprint.iacr.org/2017/504>. Citado na página 46.

COSTELlO, C.; JAO, D.; LONGA, P.; NAEHRIG, M.; RENES, J.; URBANIK, D. Efficient compression of SIDH public keys. In: Advances in Cryptology - Eurocrypt 2017. Paris, France: Springer, 2017. (Lecture Notes in Computer Science, 10210), p. 679-706. Citado 19 vezes nas páginas $16,18,23,24,54,55,58,59,60,61,62,63,65,68,72,77,78,82$ e 83.

COSTELLO, C.; LONGA, P.; NAEHRIG, M. Efficient algorithms for supersingular isogeny Diffie-Hellman. In: Advances in Cryptology - Crypto 2016. Santa Barbara (CA), USA: Springer, 2016. (Lecture Notes in Computer Science, 9814), p. 572-601. Citado na página 22.

COSTELLO, C.; SMITH, B. Montgomery curves and their arithmetic. Journal of Cryptographic Engineering, Springer, p. 1-14, 2017. Citado na página 95.

COUVEIGNES, J. M. Hard homogeneous spaces. 1997. Unpublished manuscript (1997). Updated version: Cryptology ePrint Archive, Report 2006/291 (2006). <https: //eprint.iacr.org/2006/291>. Citado na página 21.

DeFEO, L.; JAO, D.; PLÛT, J. Towards quantum-resistant cryptosystems from supersingular elliptic curve isogenies. Journal of Mathematical Cryptology, De Gruyter, v. 8, n. 3, p. 209-247, 2014. Citado 6 vezes nas páginas 18, 21, 45, 51, 74 e 75. 
DIFFIE, W.; HELLMAN, M. E. New directions in cryptography. IEEE Transactions on Information Theory, IEEE, v. 22, p. 644-654, 1976. Citado na página 43.

DOLISKANI, J.; PEREIRA, G. C. C. F.; BARRETO, P. S. L. M. Faster Cryptographic Hash Function From Supersingular Isogeny Graphs. 2017. Cryptology ePrint Archive, Report 2017/1202. <https://eprint.iacr.org/2017/1202>. Citado na página 18.

FEO, L. D.; GALBRAITH, S. D. Seasign: Compact isogeny signatures from class group actions. In: ISHAI, Y.; RIJMEN, V. (Ed.). Advances in Cryptology - EUROCRYPT 2019. Cham: Springer International Publishing, 2019. p. 759-789. Citado na página 85.

FREY, G.; MüLLER, M.; RüCK, H. The Tate pairing and the discrete logarithm applied to elliptic curve cryptosystems. IEEE Transactions on Information Theory, v. 45, n. 5, p. 1717-1719, 1999. Citado 2 vezes nas páginas 41 e 65.

FREY, G.; RüCK, H. G. A remark concerning $m$-divisibility and the discrete logarithm problem in the divisor class group of curves. Mathematics of Computation, v. 62, p. 865-874, 1994. Citado na página 42.

GALBRAITH, S.; HARRISON, K.; SOLDERA, D. Implementing the Tate pairing. In: Algorithm Number Theory Symposium - ANTS V. Sydney, Australia: Springer, 2002. (Lecture Notes in Computer Science, v. 2369), p. 324-337. Citado na página 41.

GALBRAITH, S.; STOLBUNOV, A. Improved Algorithm for the Isogeny Problem for Ordinary Elliptic Curves. 2011. Citado na página 21.

GALBRAITH, S. D.; PETIT, C.; SHANI, B.; TI, Y. B. On the security of supersingular isogeny cryptosystems. In: Advances in Cryptology - Asiacrypt 2016. Hanoi, Vietnam: Springer, 2016. (Lecture Notes in Computer Science, 10031), p. 63-91. Citado na página 24.

HUSEMÖLLER, D. Elliptic Curves. 2nd. ed. New York, USA: Springer, 2004. v. 111. (Graduate Texts in Mathematics, v. 111). Citado 2 vezes nas páginas 56 e 57.

IBM. IBM Scientists Achieve Critical Steps to Building First Practical Quantum Computer. 2015. <https://www-03.ibm.com/press/us/en/pressrelease/46725.wss >. Acessado em 24 de novembro de 2020. Citado na página 22.

INTEL. Intel Invests US\$ 50 Million to Advance Quantum Computing. 2015. <https://newsroom. intel.com/news-releases/intel-invests-us50-million-to-advance-quantum-computing/\#gs. lourwy $>$. Acessado em 24 de novembro de 2020. Citado na página 22.

JAO, D.; DeFEO, L. Towards quantum-resistant cryptosystems from supersingular elliptic curve isogenies. In: Post-Quantum Cryptography - PQCrypto 2011. Taipei, Taiwan: Springer, 2011. (Lecture Notes in Computer Science, 7071), p. 19-34. Citado 3 vezes nas páginas 21, 45 e 51.

JAQUES, S.; SCHANCK, J. M. Quantum cryptanalysis in the RAM model: Claw-finding attacks on SIKE. 2019. Cryptology ePrint Archive, Report 2019/103. <https://eprint.iacr.org/2019/103>. Citado na página 45.

JOUX, A.; NGUYEN, K. Separating Decision Diffie-Hellman from Diffie-Hellman in Cryptographic Groups. 2001. Cryptology ePrint Archive, Report 2001/003. <http: //eprint.iacr.org/2001/003>. Citado na página 41. 
KELLY, J.; BARENDS, R.; FOWLE, A. A step closer to quantum computation with Quantum Error Correction. 2015. < https://ai.googleblog.com/2015/03/ a-step-closer-to-quantum-computation.html > . Acessado em 24 de novembro de 2020. Citado na página 21.

KIRKWOOD, D.; LACKEY, B. C.; MCVEY, J.; MOTLEY, M.; SOLINAS, J. A.; TULLER, D. Failure is not an Option: Standardization Issues for Post-Quantum Key Agreement. 2015. Workshop on Cybersecurity in a Post-Quantum World. Citado na página 24.

KOBLITZ, N. A Course in Number Theory and Cryptography. 2nd. ed. Berlin, Heidelberg: Springer-Verlag, 1994. (Graduate Texts in Mathematics, 114). Citado na página 34.

KOZIEL, B.; JALALI, A.; AZARDERAKHSH, R.; KERMANI, M. M.; JAO, D. NEON-SIDH: Efficient Implementation of Supersingular Isogeny Diffie-Hellman Key-Exchange Protocol on ARM. 2016. Cryptology ePrint Archive, Report 2016/669. <https://eprint.iacr.org/2016/669>. Citado na página 23.

LIDZBORSKI, N. Staying at the forefront of email security and reliability: HTTPS-only and 99.978 percent availability. 2014. <https://googleblog.blogspot.com/2014/03/ staying-at-forefront-of-email-security.html> . Acessado em 4 de agosto de 2019. Citado na página 15.

MENEZES, A. J. Elliptic Curve Public Key Cryptosystems. [S.1.]: Kluwer Academic Publishers, 1993. Citado na página 38.

MENEZES, A. J.; OKAMOTO, T.; VANSTONE, S. A. Reducing elliptic curve logarithms to logarithms in a finite field. IEEE Transactions on Information Theory, IEEE, v. 39, p. 1639-1646, 1993. Citado na página 41.

Microsoft SIDH team. SIDH v2.0. 2017. < https://www.microsoft.com/en-us/research/project/ sidh-library/>. Citado na página 81.

MILLER, V. Elliptic curves and their use in cryptography. Waterloo, Canada: [s.n.], 1998. Elliptic Curve Cryptography Workshop - ECC'98. Citado na página 41.

MONTGOMERY, P. L. Speeding the Pollard and elliptic curve methods of factorization. Mathematics of Computation, v. 48, n. 177, p. 243-264, 1987. Citado 3 vezes nas páginas 38, 61 e 65.

NAEHRIG, M.; RENES, J. Dual isogenies and their application to public-key compression for isogeny-based cryptography. In: GALBRAITH, S. D.; MORIAI, S. (Ed.). Advances in Cryptology-ASIACRYPT 2019. Cham: Springer International Publishing, 2019. p. 243-272. ISBN 978-3-030-34621-8. Citado na página 85.

NIST. Workshop on Cybersecurity in a Post-Quantum World. 2015. <https://csrc.nist.gov/ publications/detail/nistir/8105/final>. Acessado em 24 de novembro de 2020. Citado na página 22.

PEREIRA, G.; DOLISKANI, J.; JAO, D. x-only point addition formula and faster compressed sike. Journal of Cryptographic Engineering, p. 1-13, 11 2020. Citado na página 85.

POHLIG, S. C.; HELLMAN, M. E. An improved algorithm for computing logarithms over $\mathrm{GF}(p)$ and its cryptographic significance. IEEE Transactions on Information Theory, IEEE, v. 24, n. 1, p. 106-110, 1978. Citado 2 vezes nas páginas 54 e 72. 
ROSTOVTSEV, A.; STOLBUNOV, A. Public-key cryptosystem based on isogenies. 2004. Master's thesis (2004). Updated version: Cryptology ePrint Archive, Report 2006/145 (2006). $<$ https://eprint.iacr.org/2006/145>. Citado na página 21.

SCHAEFER, E.; STOLL, M. How to do a $p$-descent on an elliptic curve. Transactions of the American Mathematical Society, v. 356, n. 3, p. 1209-1231, 2004. Citado 2 vezes nas páginas 18 e 60.

SHOR, P. W. Algorithms for quantum computation: Discrete logarithms and factoring. In: Proceedings of the 35th Annual Symposium on Foundations of Computer Science. Washington, DC, USA: IEEE Computer Society, 1994. (SFCS '94), p. 124-134. Citado 2 vezes nas páginas 15 e 21.

SHOUP, V. A computational introduction to number theory and algebra. Cambridge (UK): Cambridge University Press, 2005. Citado 2 vezes nas páginas 74 e 77.

SIKE. Supersingular isogeny key encapsulation. 2017. < https://sike.org/>. Acessado em 24 de novembro de 2020. Citado na página 24.

SILVERMAN, J. H. The Arithmetic of Elliptic Curves. Berlin, Germany: Springer-Verlag, 1986. (Graduate Texts in Mathematics, 106). Citado 6 vezes nas páginas 31, 41, 44, 45, 57 e 65.

SPIEGEL, M. R.; LIU, J. Mathematical Handbook of Formulas and Tables. 2nd. ed. New York, USA: McGraw-Hill, 1999. (Schaum's Outline Series). Citado na página 53.

STOLBUNOV, A. Constructing public-key cryptographic schemes based on class group action on a set of isogenous elliptic curves. Adv. Math. Commun., v. 4, n. 2, p. 215-235, 2010. Citado na página 21.

SVORE, K. The quantum quest at Microsoft. 2015. <https://www.microsoft.com/en-us/research/ blog/the-quantum-quest-at-microsoft/> . Acessado em 24 de novembro de 2020. Citado na página 22.

WASHINGTON, L. C. Elliptic Curves: Number Theory and Cryptography, Second Edition. 2. ed. [S.1.]: Chapman and Hall/CRC, 2008. ISBN 9781420071467. Citado 2 vezes nas páginas 40 e 47.

ZANON, G. H. M.; JR, M. A. S.; PEREIRA, G. C. C. F.; DOLISKANI, J.; BARRETO, P. S. L. M. Faster isogeny-based compressed key agreement. In: International Workshop on Post-Quantum Cryptography - PQCrypto 2018. Fort Lauderdale (FL), US: Springer, 2018. (Lecture Notes in Computer Science, v. 10786), p. 248-268. Citado na página 85.

ZANON, G. H. M.; JR, M. A. S.; PEREIRA, G. C. C. F.; DOLISKANI, J.; BARRETO, P. S. L. M. Faster key compression for isogeny-based cryptosystems. IEEE Transactions on Computers, v. 68, n. 5, p. 688-701, 2019. Citado na página 85. 

Apêndices 



\section{APÊNDICE A - Aritmética de Montgomery}

Apresenta-se aqui um sumário das operações aritméticas em curvas elípticas no modelo de Montgomery. Segue-de de perto a notação adotada na resenha recente de Costello e Smith (COSTELLO; SMITH, 2017).

A multiplicação por escalar (Algoritmos 12 e 13) emprega duas funções XADD (Algoritmo 14) para adição diferencial e xDBL (Algoritmo 15) para pseudo-duplicação de ponto, além da permutação condicional isócrona de variáveis XSWP (Algoritmo 16) para garantir uniformidade de acesso à memória e evitar certos ataques de canal colateral. Por motivos históricos, este processo chama-se escada de Montgomery.

Algoritmo 12 Escada (estendida) de Montgomery, $\mathrm{ML}^{+}(k, \pm P)$

INPUT: $k=\sum_{i=0}^{\ell} k_{i} 2^{i}$ with $k_{\ell}=1$, and $\pm P=\left(X_{P}: Z_{P}\right) \in(\mathfrak{5}$.

Output: $\pm[k] P=\left(X_{k}: Z_{k}\right) \in \mathbb{b}$ if $\pm P \neq O$, otherwise $Z_{k}=0$; and also $\pm[k+1] P=\left(X_{k+1}\right.$ : $\left.Z_{k+1}\right) \in\left(\mathfrak{5}\right.$ if $\pm P \neq O$, otherwise $Z_{k+1}=0$.

CosT: $\ell$ chamadas a XADD, $\ell+1$ chamadas a XDBL, $\ell+1$ chamadas a xSWP.
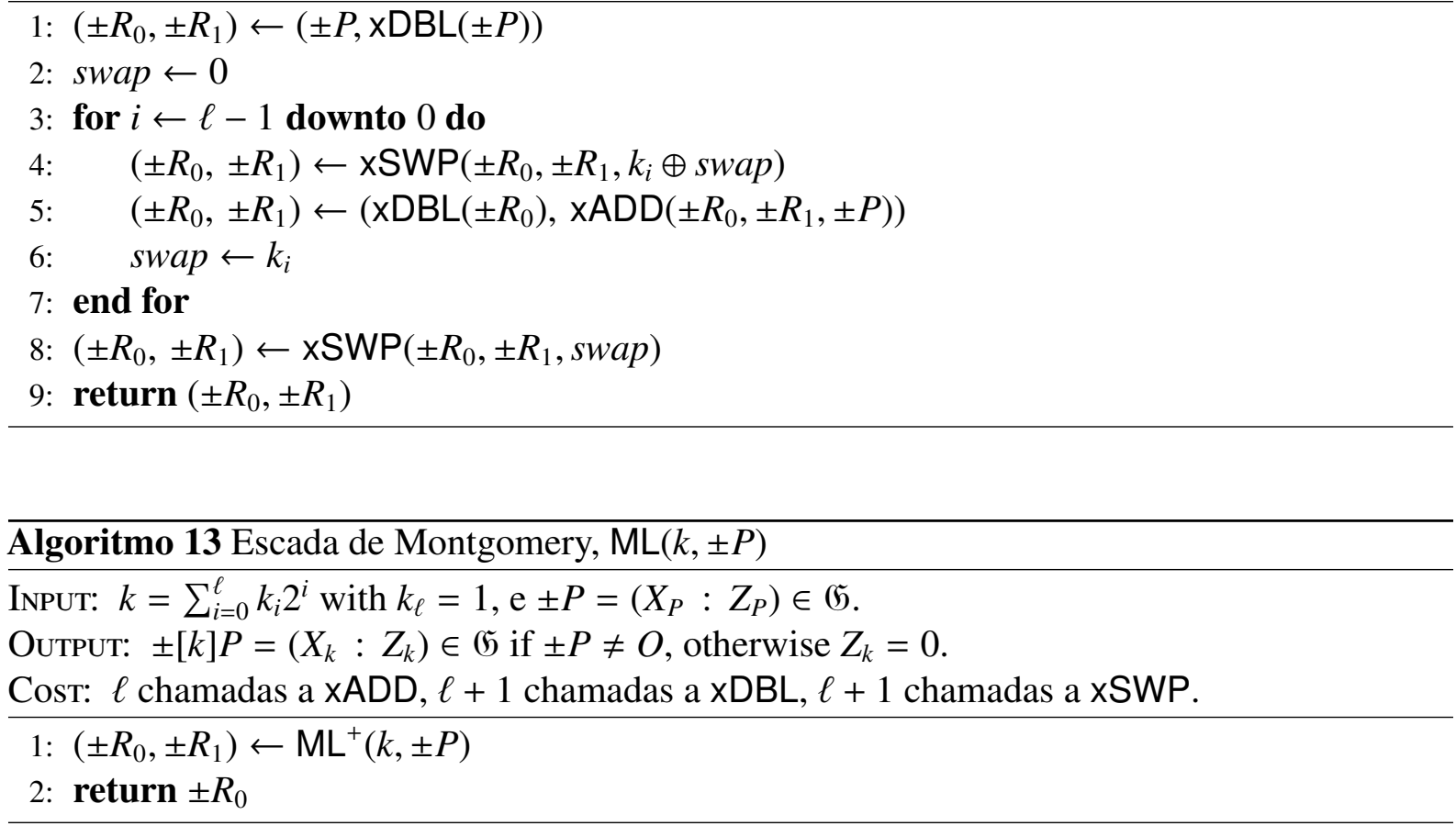

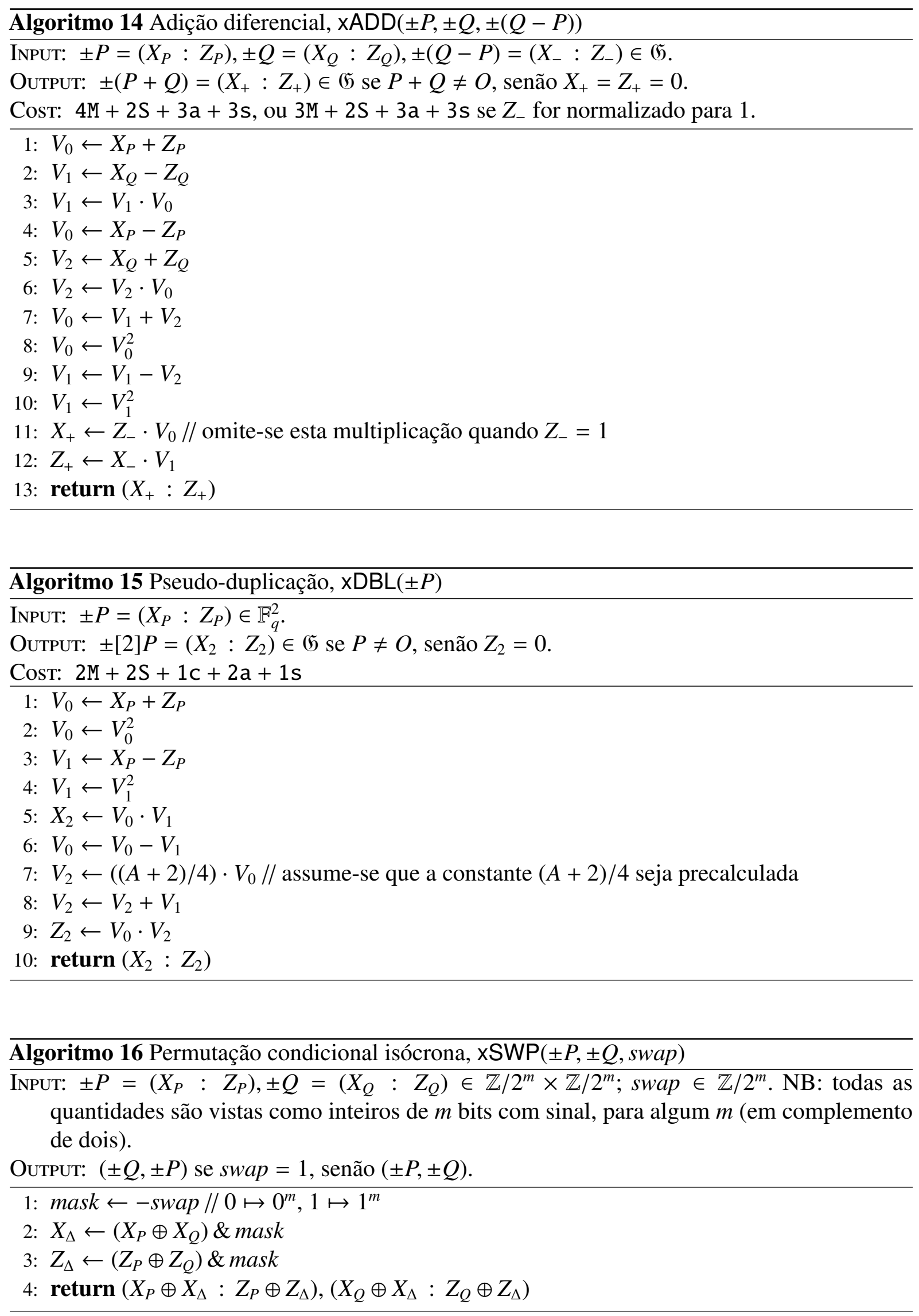